\title{
Prioritizing management options for conservation, with applications in avian landscape ecology
}

\author{
By: Adam Clark Smith
}

\begin{abstract}
A thesis submitted to
the Faculty of Graduate Studies and Research
\end{abstract}

in partial fulfillment of

the requirements for the degree of

\section{Doctor of Philosophy}

\author{
Department of Biology \\ Carleton University \\ Ottawa, Ontario \\ May 14, 2010
}

(C)2010, Adam C. Smith 


\section{Library and Archives}

Canada

Published Heritage

Branch

395 Wellington Street

Ottawa ON K1A ON4

Canada
Bibliothèque et

Archives Canada

Direction du

Patrimoine de l'édition

395, rue Wellington

Ottawa ON K1A 0N4

Canada
Your file Votre référence

ISBN: 978-0-494-67878-7

Our file Notre référence

ISBN: 978-0-494-67878-7
NOTICE:

The author has granted a nonexclusive license allowing Library and Archives Canada to reproduce, publish, archive, preserve, conserve, communicate to the public by telecommunication or on the Internet, loan, distribute and sell theses worldwide, for commercial or noncommercial purposes, in microform, paper, electronic and/or any other formats.

The author retains copyright ownership and moral rights in this thesis. Neither the thesis nor substantial extracts from it may be printed or otherwise reproduced without the author's permission.
AVIS:

L'auteur a accordé une licence non exclusive permettant à la Bibliothèque et Archives Canada de reproduire, publier, archiver, sauvegarder, conserver, transmettre au public par télécommunication ou par l'Internet, prêter, distribuer et vendre des thèses partout dans le monde, à des fins commerciales ou autres, sur support microforme, papier, électronique et/ou autres formats.

L'auteur conserve la propriété du droit d'auteur et des droits moraux qui protège cette thèse. $\mathrm{Ni}$ la thèse ni des extraits substantiels de celle-ci ne doivent être imprimés ou autrement reproduits sans son autorisation.
In compliance with the Canadian Privacy Act some supporting forms may have been removed from this thesis.

While these forms may be included in the document page count, their removal does not represent any loss of content from the thesis.
Conformément à la loi canadienne sur la protection de la vie privée, quelques formulaires secondaires ont été enlevés de cette thèse.

Bien que ces formulaires aient inclus dans la pagination, il n'y aura aucun contenu manquant. 
Abstract:

To prioritize competing conservation management options ecologists and conservation biologists must estimate their relative potential benefits to biodiversity. Quantitative estimates of these relative potential benefits, or similarly, the relative effects of ecological factors on biodiversity, are uncommon in the literature. In addition, studies have used a wide range of disparate statistical methods, which has limited our ability to synthesize their results. In this thesis, I address the following questions: 1) Does our current understanding of the relative effects of habitat loss and habitat fragmentation on biodiversity depend on the choice of statistical methods used to account for their correlation; and which methods are the least biased by this collinearity? 2) Are the relative effects of habitat amount, habitat fragmentation, and matrix quality scale dependent? 3) Of the disparate statistical metrics that might be used measure the relative importance of conservation management options, what are the theoretical connections among them and which of them meet five criteria that are necessary to compare results across studies? 4) What is the relative importance of managing residential vegetation (yards and street trees) versus non-residential vegetation (parks and large greenspace) on the species richness and conservation value of an urban bird community?

In Chapter 2, I have shown that, except for standardized partial regression coefficients, the statistical methods used to account for the correlation between habitat amount and fragmentation are biased by both collinearity and the direction of their effects on biodiversity. In Chapter 3, I have shown a scale dependency in the relative effects of forest amount and forest fragmentation on bird species that are considered sensitive to forest fragmentation, which is largely due to the higher variability in fragmentation 
effects across scales. In Chapter 4, I have shown that the best statistical metric for prioritizing competing management options is a partial regression coefficient, re-scaled as a proportion of the practical or theoretical limits to management. I have also proposed a theoretical framework and categorization of the statistical metrics of relative importance as well as practical criteria by which to judge other metrics. In Chapter 5, I have shown that the relative effects of residential and non-residential vegetation area on the richness and conservation value of an urban bird community are approximately equal at a local scale, but as human population density increases, the relative importance of non-residential vegetation declines. 


\title{
Acknowledgements
}

\author{
I dedicate this thesis to: \\ Peter Clark Smith-Moores, Leo Barrett Smith-Moores, \\ and Daisy Elizabeth Moores \\ for perspective and everything else
}

I have learned so much from my co-supervisors, Lenore and Charles. I am particularly indebted to you for: your friendship, your guidance, your patience with a $\mathrm{PhD}$ thesis that often must have seemed like a random walk along a series of questionable tangents, and most important, the honour of your unwaveringly keen criticism. Your mentorship sets a goal to which I aspire.

I am also grateful to the third member of my committee, David Currie, for many stimulating philosophical discussions over the years. I am fortunate to have had Scott Mitchell contribute his expertise to my examination committee; and, I want to especially thank Kevin McGarigal for his thoughtful criticism of, and appreciation for, my work. I look forward to future collaborations and discussions.

I owe a large debt to the volunteer naturalists who donate their time, money, and expertise to the Ottawa Breeding Bird Count and the Ontario Breeding Bird Atlas. Thanks to the official sponsors of the Ontario Breeding Bird Atlas (Bird Studies Canada, Canadian Wildlife Service, Federation of Ontario Naturalists, Ontario Field Ornithologists, and Ontario Ministry of Natural Resources) for supplying Atlas data, and to the thousands of volunteer participants who gathered data for the project. Funding for my work was provided by an NSERC, PGS-D scholarship and by Environment Canada, through Charles Francis. 
Jeremy Kerr, Matt Betts, and Roland Thomas provided insightful comments on Chapters 3 and 4. Will Turner, Rachel McCaffrey, and Peter Blancher provided important help in establishing the Ottawa Breeding Bird Count. Ed Bruggink, Marija Gojmerac, Joan Mallett, Anna Riethman-Gladish, Tom Sherratt, Nigel Waltho and Mike Weber have made my time as a TA and a Carleton student a real pleasure.

I will always cherish my time in the GLEL and I owe a tremendous thanks to the directors, particularly Lenore, to Dan Bert, and to all of the other students and extended lab-members. The combined expertise, collegial atmosphere, and lunch-time discussions at the chalk-board have been instrumental in almost every aspect of my thesis. Even more importantly, Friday discussions, canoe-trips, beers at Mike's Place, and numerous impromptu opportunities for procrastination have made coming to work an unmitigated pleasure. In particular, I must thank Elysia Brunet, Brian Campbell, Glenn Cunnington, Laura Dingle-Robertson, Felix Eigenbrod, Jude Girard, Leif Olson, Dave Omond, Jon Pasher, Raphael Proulx, Pauline Quesnelle, Trina Rytwinski, Lutz Tischendorf, and Rebecca Tittler for invaluable help at many points during my time here. Although not an official GLEL member, I also want to thank my good friend Isabelle Verreault for listening attentively, at the end of many a long day, to the daily trials and tribulations of "Deep Thought".

I am also grateful to four people for their informal mentorship: to John Virgl of Golder Associates, Saskatoon, for encouraging me to begin this process; to Lutz Tischendorf for guidance and help with many different parts of my thesis and with the Ottawa Breeding Bird Count; to Jean-Louis Martin for his friendship, canoe-trip stories, and for nurturing my scientific self-confidence; and finally, to Nicola Koper at the 
University of Manitoba, for her collaborative enthusiasm that opened the door to my first "Eureka" moment.

I am tremendously grateful to my family for their support. Mom, Dad, Alex, Laura, Joan, Ted, Jen, Alison, and Robert; your unwavering interest and enthusiasm in my rather obscure, statistical, and extremely geeky approach to "studying birds" has been immeasurably important. Peter and Leo; I appreciate your patience with your father's divided attention over this last 6 months and I cannot express how much strength, stamina, and perspective I have gained from your sublime presence.

Finally, to Daisy; I am enormously fortunate. Without your friendship, intelligence, humour, strength, beauty, stamina, patience, and Goddess-like maternal prowess, this thesis and this life would be profoundly diminished. 


\section{Table of Contents}

Abstract:

Acknowledgements .....................................................................................................

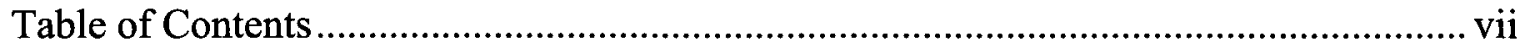

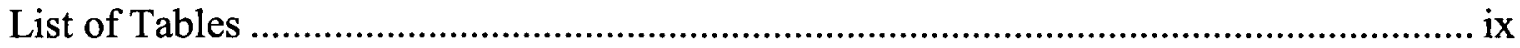

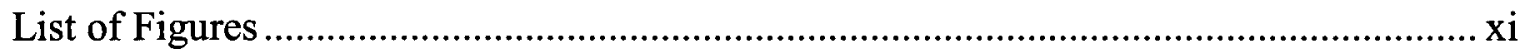

Chapter 1. General Introduction ............................................................................ 1

Chapter 2. Confronting collinearity: Comparing methods for disentangling the effects of habitat loss and fragmentation .................................................................................. 9

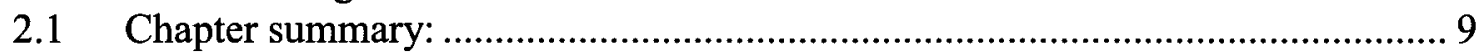

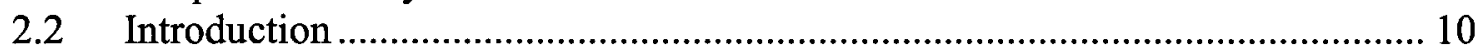

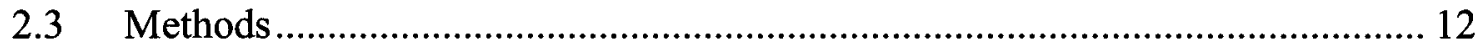

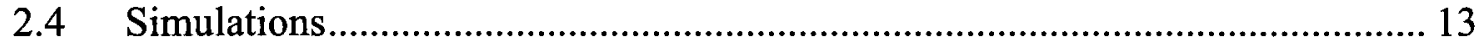

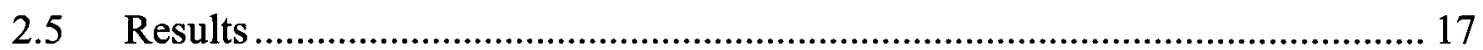

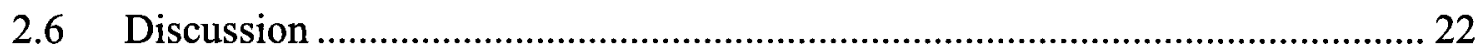

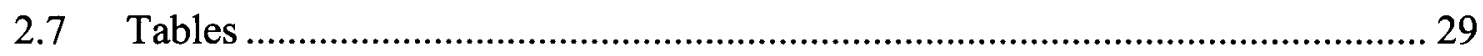

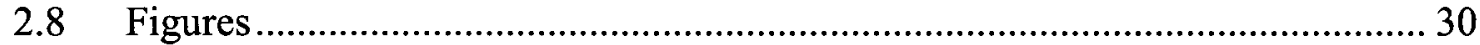

Chapter 3. Landscape size affects the relative importance of habitat amount, habitat fragmentation, and matrix quality on forest birds............................................................ 37

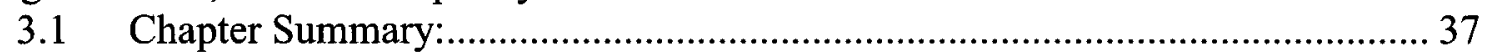

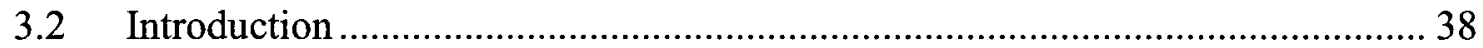

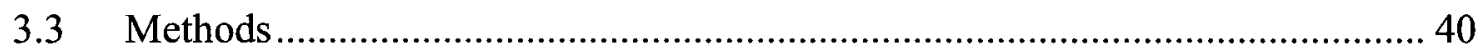

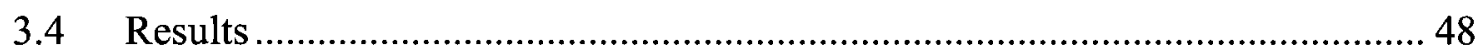

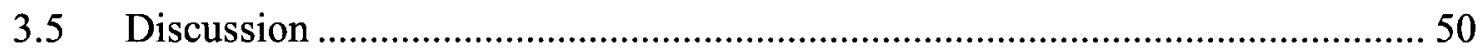

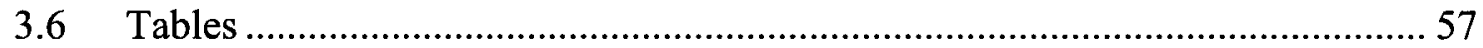

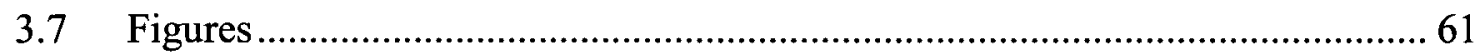

Chapter 4. How should we predict the relative impacts of management options in

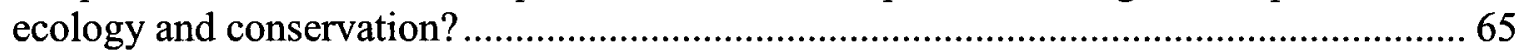

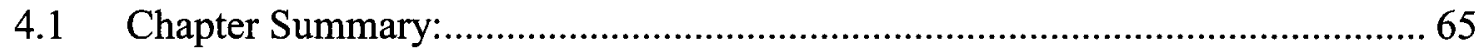

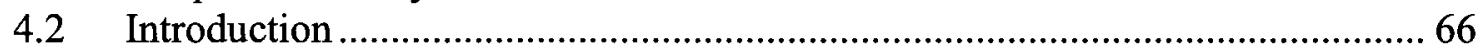

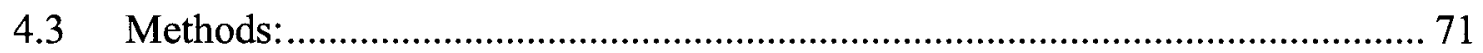

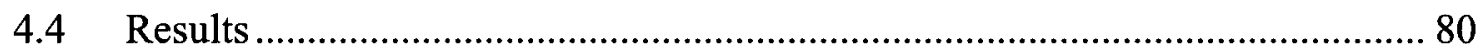

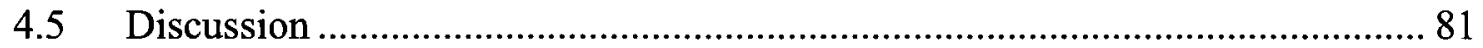

Recommendations for estimating relative importance in conservation ........................... 88

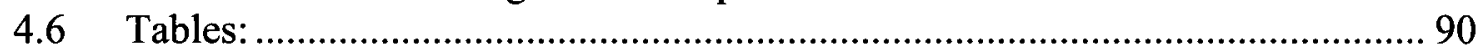

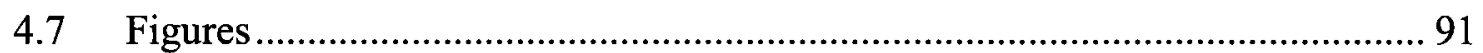

Chapter 5. Residential vegetation can be just as important as parks and greenspace for

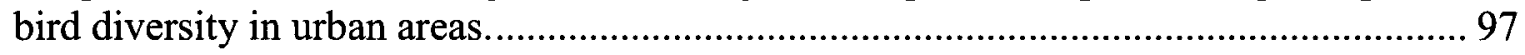

5.1 Chapter Summary:...................................................................................... 97

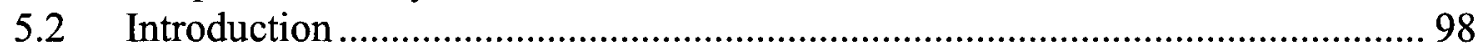

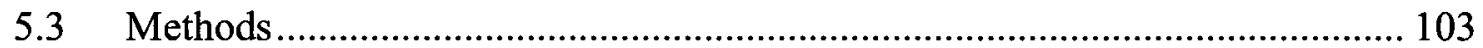

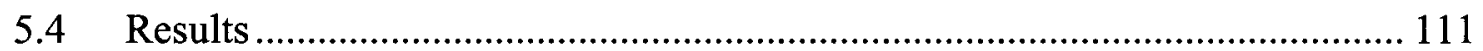

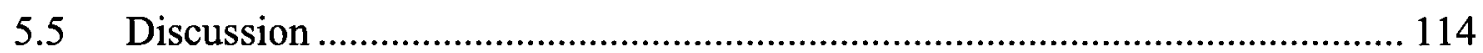

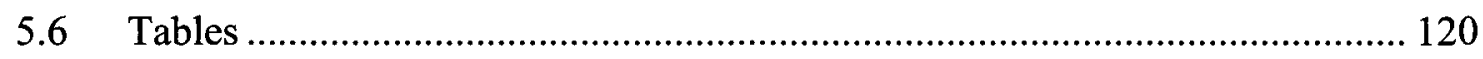

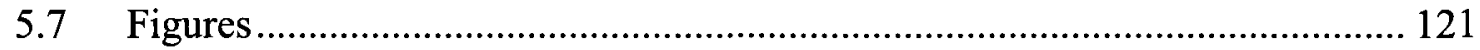

Chapter 6. General Conclusion................................................................................ 126 


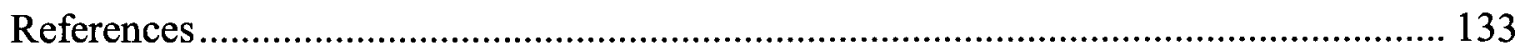
Appendix I 


\section{List of Tables}

Table 2.1. Summary of results and the statistical methods used in studies that have measured the relative effects of habitat loss and fragmentation.

Table 3.1. Species with previously demonstrated, significantly negative responses to increasing forest fragmentation, independent of the effects of habitat loss. *Four species showed significantly negative responses in more than one study.

Table 3.2. Standardized coefficients from the most parsimonious model (lowest AIC) within landscapes of 5 different sizes. Predictors included 3 landscape variables total amount of forest (Forest), the proportion of forest area within the core of large forests (Core), and the density of roads (Road) - plus the Forest*Core interaction. Models predicted the number of fragmentation sensitive species observed (Poisson regression) and the occurrence (logistic regression) of Ovenbird and Veery at 2951 forested point counts in Southern Ontario Canada. Candidate models included all 8 possible combinations of the 3 landscape predictors, plus the Forest*Core interaction. All models included covariates controlling for spatial autocorrelation, time of day, season, habitat heterogeneity, and roadside survey biases.

Table 3.3. Summed Akaike weights for the 3 landscape predictors - total amount of forest (Forest), the proportion of forest area within the core of large forests (Core), and the density of roads (Road), plus the Forest*Core interaction - measured within landscapes of 5 different sizes. Models predicted the number of fragmentation sensitive species observed (Poisson regression) and the occurrence (logistic regression) of Ovenbird and Veery at 2951 forested point counts in Southern Ontario, Canada. Weights were summed across the $95 \%$ confidence set of supported models from among the same competing models as in Table 3.2

Table 3.4. Collinearity among the landscape predictors increased with landscape size. Variance inflation factors (VIF) and pair-wise Pearson correlation coefficients for predictors: total amount of forest (Forest), the proportion of forest area within the core of large forests (Core), and the density of roads (Road). VIF represents the predictor's overall collinearity in a regression model with all other landscape predictors, the interaction between Forest and Core, and all covariates (see Table 3.2 for a list of covariates). Values above the diagonal are for large landscapes (10 $\mathrm{km}$ radius) and below are for small landscapes ( $0.5 \mathrm{~km}$ radius). Values for intermediate scales are intermediate to those shown here.

Table 4.1. Relative importance metrics compared in this study and/or identified in my literature review.

Table 5.1. Correlations among the key predictors of urban bird richness. Values above the diagonal are for the full dataset $(n=247)$, those below the diagonal are for a subsample of points chosen to reduce the correlation between VegNonRes 75 and VegRes75. Values in parentheses are the variance inflation factors for each predictor in the full model (i.e., 1/(1-R2) of a linear regression of each predictor on all other predictors). Variable names indicate either vegetation area ("Veg") or percent of vegetation that is trees and shrubs ("pWood"), followed by modifiers indicating residential or non-residential vegetation and the scale $(75=$ local, $425=$ landscape). The pattern of pairwise correlations and VIF for pShrub variables, used 
to model shrub-successional species richness, are similar to those for $\mathrm{pWood}$ variables. 


\section{List of Figures}

Figure 2.1. Correlation matrix of standardized landscape predictor variables $(n=350)$. Numbers in parentheses are the variance inflation factors for each predictor, and numbers below the diagonal are Pearson correlation coefficients for all predictor pairs

Figure 2.2. Partial regression coefficients from three statistical methods were unbiased estimates of effect strength, even with highly correlated predictor variables. Estimates were unbiased for - 1) multiple regression (A, D), 2) the best model selected using AIC in a step-wise variable selection $(\mathrm{B}, \mathrm{E})$, and 3 ) the $\mathrm{MMI}$ averaged coefficients $(\mathrm{C}, \mathrm{F})$ - when the influential predictors were available (i.e., correct predictors A-C or too many predictors D-F). Points and error bars indicate the mean partial regression coefficients and $95^{\text {th }}$ percentiles from 100 replications of simulated $Y$ data. True $Y$ indicates the influential predictors (see text for definition). Horizontal dotted lines indicate the true effect strength and any arrows show deviations of the means from it. \%sig indicates the percent of replications where each predictor's regression coefficient was significantly different from zero $(p<0.05), \%$ incl indicates the percent of replications where each predictor was included in the best model, and \% excl indicates the percent of replications where each predictor was excluded from at least one of the supported models (i.e., $\triangle$ AIC $<4)$

Figure 2.3. Partial regression coefficients were biased if influential predictors were missing from the model and the strength and direction of bias depended on suppressor relationships. In A, the missing influential predictors were non-suppressors and therefore the most highly correlated predictor (Amount) was overestimated. In B, one of the missing influential predictors (Edge) was a suppressor and therefore the most highly correlated predictor (Amount) was underestimated. Partial regression coefficients from a multiple regression are shown here but the same bias applied to all three coefficientbased estimates included in Figure 2.2. See Figure 2.2 for explanation of text and symbols in the plot.

Figure 2.4. Residual regression is biased but the bias depends on suppressor effects. With suppressor effects (A), both amount and fragmentation effects are underestimated and the analysis is biased towards the residual predictor whether fragmentation (left side of A) or amount (right side of A). When there is no suppressor relationship (B) the analysis is biased towards the intact predictor. The prefix "R." indicates the residual predictor in each of the 4 separate analyses. See Figure 2.2 for explanation of text and symbols. .... 33

Figure 2.5. Summed Akaike weights overestimated the importance of uninfluential predictors (B) and in some replications, underestimated the importance of influential predictors that were more highly correlated (A). Text below each figure indicates range in the number of supported models (i.e., $\triangle \mathrm{AIC}<4$ ) across 100 replications and the percent of replications for which more than 1 model was supported. Points and error bars indicate the mean summed Akaike weights and $95^{\text {th }}$ percentiles from 100 replications of simulated $Y$ data. See Figure 2.2 for explanation of other text and symbols.

Figure 2.6. Independent variance partitions were biased estimates of effect strength for all true models in all conditions (Classical, A - D and HVP, E and F). Classical variance partitioning underestimated the effects of influential predictors (A and $B$ ) but was unbiased for uninfluential predictors (B). If missing influential predictors were not suppressors, the effects of some predictors were overestimated (C) and when missing 
influential predictors were suppressors the effects of the remaining predictors were underestimated (D and Edge in C). HVP estimates were biased for influential and uninfluential predictors $(\mathrm{E}$ and $\mathrm{F}$ ) and were particularly biased against suppressors (Edge in E). Points and error bars indicate the mean and 95th percentiles from 100 replications of simulated $\mathrm{Y}$ data of the percent of the variation explained by the model that is independently attributed to each predictor. See Figure 2.2 for explanation of text and symbols.

Figure 2.7. The bias in residual regression depends on suppressor effects (i.e., the correlation between amount and fragmentation and whether the fragmentation measure has a positive or negative effect on the response). This figure shows the qualitative bias in published studies that used residual regression to create fragmentation metrics orthogonal to habitat amount. The strength of the bias is directly related to the magnitude of correlation between the original predictors in the published study. If habitat loss was measured instead of amount a negative effect of loss on the response would be expected. In this case, switching the contents of boxes A and B would show the resulting bias.

Figure 3.1. Distribution of forest cover (grey areas) and 2951 point count sampling stations with $>95 \%$ forest cover inside the $100 \mathrm{~m}$ radius sampling area (crosses), within the study area (portion of Bird Conservation Region 13 in Ontario, Canada).

Figure 3.2. Poisson regressions coefficients for the effects of the total amount of forest (Forest), the proportion of forest area in large core forests (Core), and the density of roads (Road) in landscapes of 5 different radii on the number of fragmentation sensitive bird species observed at forested point counts in Southern Ontario Canada. Points are the standardized, averaged coefficients (weighted by the model's Akaike weight) from all models in a 95\% confidence set and error bars are the associated unconditional confidence limits. An interaction between Forest and Core, is demonstrated by the error bars without points. These error bars represent the confidence limits around the coefficient for Forest at low and high levels of Core $(-1$ and +1 standard deviation of Core) and for Core at low and high levels of Forest ( -1 and +1 standard deviation of Forest). All models accounted for the effects of spatial autocorrelation, time of day, season, surrounding habitat diversity, and roadside survey biases

Figure 3.3. Logistic regressions coefficients for the effects of the total amount of forest (Forest), the proportion of forest area in large core forests (Core), and the density of roads (Road) in landscapes of 5 different radii on the occurrence of Ovenbird at forested point counts in Southern Ontario Canada. Points are the standardized, averaged coefficients (weighted by the model's Akaike weight) from all models in a 95\% confidence set and error bars are the associated unconditional confidence limits. An interaction between Forest and Core, is demonstrated by the error bars without points. These error bars represent the confidence limits around the coefficient for Forest at low and high levels of Core ( -1 and +1 standard deviation of Core) and for Core at low and high levels of Forest $(-1$ and +1 standard deviation of Forest). All models accounted for the effects of spatial autocorrelation, time of day, season, surrounding habitat diversity, and roadside survey biases.

Figure 3.4. Logistic regressions coefficients for the effects of the total amount of forest

(Forest), the proportion of forest area in large core forests (Core), and the density of roads (Road) in landscapes of 5 different radii on the occurrence of Veery at forested point counts in Southern Ontario Canada. Points are the standardized, averaged coefficients 
(weighted by the model's Akaike weight) from all models in a 95\% confidence set and error bars are the associated unconditional confidence limits. An interaction between Forest and Core, is demonstrated by the error bars without points. These error bars represent the confidence limits around the coefficient for Forest at low and high levels of Core ( -1 and +1 standard deviation of Core) and for Core at low and high levels of Forest $(-1$ and +1 standard deviation of Forest). All models accounted for the effects of spatial autocorrelation, time of day, season, surrounding habitat diversity, and roadside survey bias.

Figure 4.1. Relationships between sample size and strength-, variance-, and confidence-metrics of relative importance, calculated for three orthogonal, simulated predictors with linear effects on a response (Equation 2). Each plot is arranged so that the metrics that satisfy the sample size criterion show: three non overlapping lines, rank $\mathrm{x}_{1}>\mathrm{x}_{2}>\mathrm{x}_{3}$, and are constant from left (larger samples) to right (smaller samples) along the $\mathrm{x}$-axis. Lines represent the mean estimated metric value across 1000 iterations of a random sample, drawn from an $\mathrm{N}=100000$ population of simulated data.

Figure 4.2. Relationships between collinearity among predictors and strength-, variance-, and confidence-metrics of relative importance, calculated for three simulated predictors with linear effects on a response (Equation 2). Plots are arranged so that the metrics that satisfy the collinearity criterion show: three non overlapping lines, rank $x_{1}>x_{2}>x_{3}$, and are constant from left (orthogonal variables) to right (pair-wise correlations $=0.999$ ) along the $\mathrm{x}$-axis. Lines represent the mean estimated metric value for each predictor across 1000 iterations of a random sample of size $n=500$, from an $N=100000$ population of simulated data.

Figure 4.3. Relationships between the relative variation (i.e., the proportion of the original range of $\mathrm{x}_{2}$ that was sampled) and strength-, variance-, and confidence-metrics of relative importance, calculated for three orthogonal, simulated predictors with linear effects on a response (Equation 2). Each plot is arranged so that the metrics that satisfy the relative variation criterion show: three non overlapping lines, rank $x_{1}>x_{2}>x_{3}$, and are constant from left from left (full range of $x_{2}$ ) to right $\left(2.5 \%\right.$ of the original range in $x_{2}$ sampled) along the $\mathrm{x}$-axis. Lines represent the mean estimated metric value for each predictor and the upper and lower $95^{\text {th }}$ percentiles, across 1000 iterations of a random sample of size $\mathrm{n}=500$, from an $\mathrm{N}=100000$ population of simulated data.

Figure 4.4. Relationships between the correlation of a spurious predictor $\left(\mathrm{x}_{4}\right)$ with an influential variable $\left(\mathrm{x}_{1}\right)$ and strength-, variance-, and confidence-metrics of relative importance, calculated for three simulated predictors with linear effects on a response (Equation 2) and a fourth, spurious predictor with no effect on the response $\left(\mathrm{x}_{4}\right)$. Plots are arranged so that the metrics that satisfy the spurious predictor criterion show: three non overlapping lines, rank $\mathrm{x}_{1}>\mathrm{x}_{2}>\mathrm{x}_{3}>\mathrm{x}_{4}$, and are constant from left (no correlation between $\mathrm{x}_{1}$ and $\mathrm{x}_{4}$ ) to right (pair-wise correlation between $\mathrm{x}_{1}$ and $\mathrm{x}_{4}=0.999$ ) along the $\mathrm{x}$-axis. Lines represent the mean estimated metric value for each predictor across 1000 iterations of a random sample of size $\mathrm{n}=500$, from an $\mathrm{N}=100000$ population of simulated data.

Figure 4.5. Relationships between strength of a suppressor effect and strength-, variance-, and confidence-metrics of relative importance, calculated for three simulated predictors with linear effects on a response (Equation 3). Plots are arranged so that the metrics that satisfy the suppressor predictor criterion show: three non overlapping lines, rank $\left|\mathrm{x}_{1}\right|=\left|\mathrm{x}_{2}\right|>\mathrm{x}_{3}$, and are constant from left (orthogonal variables) to right (pair-wise 
correlations $=0.999$, where the effects of $x_{1}$ and $x_{2}$ completely cancel each other) along the $\mathrm{x}$-axis. Lines represent the mean estimated metric value for each predictor, across 1000 iterations of a random sample of size $n=500$, from an $N=100000$ population of simulated data.

Figure 4.6. Framework showing the underlying connections among three categories of relative importance metrics, which are created by the dichotomy of statistical and biological significance within regression modeling. Strength-metrics measure biological significance, which is a characteristic of the underlying ecological phenomenon or the statistical population. Confidence-metrics measure statistical significance, which is a characteristic of the statistical sample. Variance-metrics measure both statistical and biological significance and are influenced by both the sample and the ecological phenomenon.

Figure 5.1. Map of study area in Ottawa, Ontario, Canada, showing the distribution of point counts across the city. Insets demonstrate how each of the four vegetation area (left inset), and four vegetation structure (percent of vegetation that is tree or shrub cover, right inset) variables were measured. See table 5.1 for a description of the variable names.

Figure 5.2. Relative effects of residential and non-residential vegetation area on four measures of bird diversity, at local (upper plots) and landscape (lower plots) scales. Points represent the partial coefficients for residential and non-residential vegetation area, scaled for a 10 unit change in the percent of the landscape made up of either residential or nonresidential vegetation, after controlling for the remaining effects in Equation 2 or, for S.Shrub, Equation 3. Error bars represent $95 \%$ confidence intervals. Numbers in parentheses are the proportion of explained deviance for the model in Equation 2 or 3.122

Figure 5.3. Relative effects of residential and non-residential vegetation structure (percent of vegetation that is wooded for S.Native, S.Forest, and CI.Rank, and the percent that is shrub or tall grass for S.Shrub) on four measures of bird diversity, at local (upper plots) and landscape (lower plots) scales. Points represent the partial coefficients, scaled for a 10 unit change in percent wooded or shrub, after controlling for the remaining effects in Equation 2 or for S.Shrub, Equation 3. Error bars represent $95 \%$ confidence intervals. Numbers in parentheses are the proportion of explained deviance for the model in Equation 2 or 3 .

Figure 5.4. Limits to residential and non-residential vegetation area with increasing human population density. Lines represent $95 \%$ quantile regression models, fit separately to nonresidential vegetation (solid line) and residential vegetation (dotted line).

Figure 5.5. Relative importance of residential and non-residential vegetation at high human population density ( 300 residents/ha). Points represent the proportional partial coefficients, scaled to a change in vegetation area that is $10 \%$ of the range within practical limits to management. The limits to management were estimated from the ranges in the areas of residential and non-residential vegetation at high human population density (i.e., the point where the lines in Figure 5.5 at a density of 300). Compare with figure 5.2. Error bars represent $95 \%$ confidence intervals. 


\section{Chapter 1. General Introduction}

Estimating relative effects of different ecological processes or competing management options is important in ecology and conservation biology (Wilson et al. 2007). Resources are limited, action is required, and so, competing options for management must be prioritized (Sutherland et al. 2004). Decisions on conservation actions are made through a cost-benefit analysis by planners, managers, politicians, and the public, (the quality of the accounting is up for debate). It falls to ecologists and conservation biologists to quantify the benefit side of the equation, i.e., the expected benefit to biodiversity from a particular management option.

There are different ways of estimating the relative effects, or potential benefit to conservation, of competing management options. In some cases manipulative experiments, where the management options are experimentally balanced, may be the optimal approach (e.g., Pullin and Knight 2009); however, similarly useful estimates can come from observational studies where the controls on confounding factors and correlations are statistical, or from studies that combine both statistical and experimental controls (e.g., mensurative experiments sensu Hurlbert 1984, McGarigal and Cushman 2002, Brennan et al. 2002). Well controlled and balanced manipulative experiments, in the spirit of Platt's (1964) strong inference, should play some role in estimating those benefits. However, the complexity of ecological systems, the difficulty of experimentally controlling all of the potentially confounding factors, and the long time scales required, make it unlikely that experiments will be sufficient on their own (McGarigal and Cushman 2002). Indeed, for sufficiently complex problems in ecology and conservation, manipulative experiments may not be ethical or possible (Hilborn and Mangel 1997); 
indeed, they may not be the best source of information for prioritizing management options, if their quantitative results do not extrapolate to relevant spatial and temporal scales, or to realistic levels of complexity (Peters 1991, Hobbs and Hilborn 2006). Indeed, even in situations where manipulative experiments are possible, a preliminary regression analysis can predict the likely outcomes and guide decisions about which experimental treatments to apply (James and McCulloch 1990). Therefore, statistical methods will continue to be the primary means by which ecologists compare competing management options for many real-world problems in ecology and conservation biology.

However, statistical controls for correlations and confounding influences within these models are fraught with difficulties and limitations (e.g., Petraitis et al. 1996, James and McCulloch 1990). First, the predictors in the statistical model must be reasonable representations of the management options. As an example, consider an analysis that compares the relative effects of habitat amount (measured in units of area or percent of a given landscape) with a principal component axis that combines numerous aspects of habitat fragmentation. This synthetic variable may not represent the management action (e.g., decreasing habitat edge density), as clearly as habitat amount (e.g., increasing habitat area); in which case, the analysis would be biased towards the effects of habitat amount. Second, the correlations among the competing management options must not be causal. If the competing options cannot be manipulated independently of each other, at least partially, then statistical controls for their correlation are inappropriate (Grace and Bollen 2005). Indeed, if they cannot be independently manipulated, the entire question of relative importance is moot. Finally, there must be evidence that the management options have causal effects on the biodiversity response. Causality is a high standard but 
practically, this evidence should involve a plausible ecological mechanism combined with previous studies (either correlative or manipulative) demonstrating an effect (Anderson et al. 2001, Johnson 2002).

Estimating relative effects and prioritizing management options is relatively rare in ecology and conservation (Stephens et al. 2007), partly because ecology has such a strong emphasis on hypothesis tests, falsification (Popper 1962), and strong inference (Platt 1964). In its strongest form, this philosophical approach holds falsification as the gold standard by which all scientific theories, hypotheses, and explanations should be judged (Popper 1962, Platt 1964). Beginning in the late 1970's, ecologists began to strongly advocate a focus on testable, falsifiable hypotheses (Strong et al. 1979, Romesburg 1981, Simberloff 1983). This theme persists and the ecological literature frequently extols the virtues of strong inference and falsification (e.g., Peters 1991, Nichols and Williams 2006, McIntire and Fajardo 2009). For example, of the 1064 articles in the Web of Science that have cited Platt (1964), more of those articles are from ecology than any other single subject area $(\sim 15 \%$ of the 1064 citations, which represents $0.7 \%$ of all the hits from a topic search for "ecology" in Web of Science).

Strong inference and falsification have their strengths but they may have been over emphasized in ecology and conservation. Certainly, there is a solid philosophical argument and a proven practical base which shows that strong inference and falsification are elegant heuristic tools, powerful methodological goals, and the optimal approach to many scientific endeavours. However, their unqualified emphasis leads to a proliferation of trivial hypotheses, tested in the context of statistical or methodological dogma. The misuse and over-use of statistical null hypothesis testing (Yoccoz 1991), generates trivial 
and inane "findings", often because a complex question is answered with a binary, yes-no answer, e.g, effects test hypotheses that posit the existence of an effect (Guthery 2008b). Similarly, falsification dogma can lead to an avoidance of important questions that do not lend themselves easily to hypothesis testing (Hilborn and Mangel 1997). Much of ecology's dogmatic tendencies can be attributed to the exclusive and hyperbolic language of Popper and Platt (e.g., "non-scientific" or "every problem in science"). Taken at facevalue, they claim that studies with goals other than to falsify a specific hypothesis are not science; and, because of the long temporal and large spatial scales of ecological systems and the difficulties in conducting replicable experiments, ecologists, on average, are probably more sensitive to this critique than their colleagues in molecular biology, for example (Platt 1964, Peters 1991, Johnson 2002).

Ecology has many situations, questions, and objectives that do not lend themselves to an approach based on falsification and strong inference, particularly in the context of prioritizing management actions. In practice, strong inference is limited by its assumption that a "clean result" (Platt 1964) is possible, and by the time required to carry out experiments (Hilborn and Ludwig 1993, Hilborn and Mangel 1997, McGarigal and Cushman 2002). In addition, the notion of falsification excludes the possibility that hypotheses may be partially true (i.e., that more than one factor exerts an influence, Quinn and Dunham 1983, Guthery 2008a) or that a single hypothesis may be true or false in different situations (e.g., different factors may impose limits on an ecological process in different times or places, Cade et al. 1999). However, the complexity in ecological systems means that different and multiple factors may have effects in different situations and it is practically important to know: which factors, in what situations, and most 
importantly, how strong an effect (Johnson 2005). In practice, almost every ecological hypothesis is supported in some contexts and falsified in others - the real question is one of relative utility (Hilborn and Mangel 1997). A more balanced approach to the philosophy of science acknowledges that much of what is useful in ecology is the estimation of effects and the uncertainty around those effects (Quinn and Dunham 1983, Hilborn and Ludwig 1993, Guthery 2008a). Studies that are exclusively focused on hypothesis tests will be less useful to conservation than those that estimate effect sizes. For example, although there may be evidence that each of a suite of competing management options has an effect on some conservation goal, it does not follow that managing all or any of those effects will be an efficient use of resources.

The relative importance of managing habitat amount and habitat fragmentation is a good example of a complex problem where statistical methods are vital to estimating their relative effects (Fahrig 2003); however, confusion around those statistical methods has, so far, hampered synthesis (Koper et al. 2007). The question of their relative effects has been debated in landscape ecology for more than 15 years (Andren 1994, Karieva 1995, Fahrig 1997). A few relevant manipulative experiments have been done but extrapolating their results to the scale of anthropogenic landscape change is difficult because they were (of necessity) conducted in simplified, small-scale microcosms (McGarigal and Cushman 2002, Fahrig 2003). Many more studies have estimated the relative effects of habitat amount and fragmentation using observational data while statistically controlling for the correlation between them. However, the relative ranking of their effects has depended on the study (Fahrig 2003) and some of the methods used to control for the correlations are biased (Koper et al. 2007). Therefore, it is unclear to what 
extent the current synthesis reflects their relative effects on biodiversity or the choice of statistics used to measure their relative effects.

Chapter 2 begins by reviewing the statistical methods that have been used to compare the effects of, control for the correlations between, habitat loss and fragmentation. By comparing these methods under identical conditions, I demonstrate the difficulty of synthesizing across studies that have used disparate statistical methods to control for the correlation. I also compare the potential biases in each method and make recommendations on the best methods to use in future studies.

Chapter 3 applies the statistical recommendations from Chapter 2 to answer the following question: Are the relative effects of habitat amount, habitat fragmentation, and matrix quality scale dependent? My hypothesis is that their relative effects depend on the correspondence between two scales: the extent of the landscape used to measure them, and the scales of the processes that are affected by each of the three factors. The scale of an ecological process determines the scale at which its effects are evident (Addicott et al. 1987). For example, fragmentation may affect large-scale processes like source-sink population dynamics or small-scale processes like individual reproductive success; the scales of the strongest relationships between fragmentation and these two processes reflect this (e.g., Tittler et al. 2006, Ries et al. 2004). Because habitat amount, habitat fragmentation, and matrix quality affect numerous ecological processes that occur at different spatial scales, their relative effects should be scale dependent.

Chapter 4 returns to the theoretical and methodological issues around estimating the relative importance of competing management options. In the last 10 years, there have been numerous, conflicting recommendations on statistical methods to estimate the 
relative importance of competing management options in ecology (MacNally 2000, Burnham and Anderson 2002, Grace and Bollen 2005, Murray and Conner 2009). However, each of these sets of recommendations has largely ignored the methods suggested by the others, and each has implicitly redefined "relative importance" within the narrow confines of a particular statistical framework. Consequently, there is no consensus in the literature on: 1) a definition of relative importance in conservation and ecology, 2) explicit criteria by which metrics of relative importance can be judged or compared, or 3) a framework to connect and categorize the various metrics. In this chapter, I propose a definition, criteria, and a framework, as well as: define a novel way to re-scale predictors and coefficients in different units so that they are directly comparable on a scale that considers the practical limits to their management; compare all of the metrics that have been previously recommended against the criteria; and make recommendations on which metrics are best.

Chapter 5 applies the recommendations from Chapter 4 to an empirical question about the relative importance of managing vegetation in residential and non-residential areas of a city. The area and structure of non-residential vegetation (parks and large greenspaces) influences the diversity and conservation value of an urban/suburban bird community (Marzluff and Ewing 2001). In addition, there is evidence that there are similar effects of the area and structure of residential vegetation (private yards and street trees, Evans et al. 2009). I compare their relative effects, both in their raw units (i.e., hectares or percent of the landscape), and in units that account for the practical limits imposed by high human population density (i.e., the novel metric from the previous chapter), which are different for residential and non-residential vegetation. I also analyze 
their effects separately at local and landscape scales to account for the different processes that each type of vegetation may affect.

The four central chapters of my thesis are linked by their focus on prioritizing competing management options for conservation. Chapters 2 and 4 deal with the methodological and theoretical challenges of estimating the relative potential benefits of competing management options in complex ecological systems. Chapters 3 and 5 apply these methodological advances to practical questions in avian ecology and conservation. 


\section{Chapter 2. Confronting collinearity: Comparing methods for disentangling} the effects of habitat loss and fragmentation

(Adapted from: Smith A. C., N. Koper, C. M. Francis, and L. Fahrig. 2009. Confronting collinearity: Comparing methods for disentangling the effects of habitat loss and fragmentation. Landscape Ecology 24: 1271-1285)

\subsection{Chapter summary:}

Estimating the relative importance of habitat loss and fragmentation is necessary to estimate the potential benefits of specific management actions and to ensure that limited conservation resources are used efficiently. However, estimating relative effects is complicated because the two processes are highly correlated. Previous studies have used a wide variety of statistical methods to separate their effects and I speculated that the published results may have been influenced by the methods used. I used simulations to determine whether, under identical conditions, the following 7 methods generate different estimates of relative importance for realistically correlated landscape predictors: residual regression, model or variable selection, averaged coefficients from all supported models, summed Akaike weights, classical variance partitioning, hierarchical variance partitioning, and a multiple regression model with no adjustments for collinearity. I found that different methods generated different rankings of the predictors and that some metrics were strongly biased. Residual regression and variance partitioning were highly biased by correlations among predictors and the bias depended on the direction of a predictor's effect (positive vs. negative). My results suggest that many efforts to deal with the correlation between amount and fragmentation may have done more harm than good. If confounding effects are controlled and adequate thought is given to the ecological mechanisms behind modeled predictors, then standardized partial regression 
coefficients are unbiased estimates of the relative importance of amount and fragmentation, even when predictors are highly correlated.

\subsection{Introduction}

Anthropogenic landscape alteration can result in both habitat loss (a reduction in the proportion of a landscape composed of suitable habitat for a focal species, commonly measured as reduced habitat amount) and habitat fragmentation (a change in the arrangement or configuration of the remaining habitat such as increased edge density, or reduced core area). Because habitat loss and fragmentation are inevitably correlated to some degree, they are often combined into a single concept - "habitat loss and fragmentation" (Ewers and Didham 2006 and 2007) or even simply "fragmentation" (e.g., Gelling et al 2007). However, in the context of landscape planning for conservation, combining these two processes may be counter-productive, because: 1) they can be managed independently to some degree (e.g., creating corridors to link existing habitat patches could have a strong effect on fragmentation but a comparatively weak effect on habitat amount); and, 2) their effects on populations and biodiversity may be different in magnitude and even direction (e.g., creating corridors may have a positive influence on a population through increased connectivity but have a concurrent negative influence through increased edge habitat in the landscape; Fahrig 2003). As a result, it is important to understand their independent effects so that management recommendations result in the most efficient and effective use of limited conservation resources (Lindenmayer and Fischer 2007, Sutherland et al 2004).

The current understanding of the relative importance of habitat loss and fragmentation is limited because of the high degree of correlation between them 
(McGarigal and Cushman 2002, Fahrig 2003). Indeed, Koper et al (2007) recently demonstrated that one of the most common statistical approaches for separating their effects - residual regression - is highly biased. The approach involves using the residuals of a regression of one correlated predictor on another as an orthogonal predictor in a classic regression framework (See Koper et al 2007 for a detailed review). If residuals are used to create a measure of fragmentation that is orthogonal to habitat amount (or vice versa), the assessment of relative importance may be inherently biased towards habitat amount (or fragmentation). Presumably, any shared effect is allocated to amount, which is usually the intact (i.e., non-residual) predictor (Freckleton 2002). In some contexts, an a priori bias may be appropriate (e.g., if habitat amount is inherently easier to manage but see Ewers and Didham 2007); however, these residual regression studies have also been incorrectly cited as evidence that amount is more important than fragmentation (e.g., in Flather and Bevers 2002; Fahrig 2003; Turner 2005).

The bias inherent in residual regression may be even more complicated than has been suggested. The presumed bias towards habitat amount assumes that the effects of habitat amount and fragmentation on biodiversity do not conflict, but they may. For example, if an increase in habitat amount has a positive effect on biodiversity but is positively correlated with a measure of fragmentation that has a negative effect, then the two processes will conflict and suppress (mask) each other's effects. Variables that have these conflicting effects (opposite qualitative effects and a positive correlation or similar qualitative effects and a negative correlation) are referred to as suppressor variables (Cohen and Cohen 1983). In these conditions, residual regression may underestimate both effects. In addition, suppressor effects likely influence other statistical methods. For 
example, if an influential suppressor variable is removed from a multiple regression, the effects of the remaining predictor will be underestimated (Legendre and Legendre 1998). These serious consequences should clearly be addressed in comparing the relative effects of habitat loss and fragmentation.

There are many statistical approaches for estimating the relative effects of correlated predictor variables such as habitat amount and fragmentation (Graham 2003, Burnham and Anderson 2002, Gromping 2007) but synthesizing the literature is difficult if previous results have been determined by the statistical methods rather than the actual biological effects. Therefore, in this paper I have three objectives: 1) to review the statistical methods that have been used to estimate the relative importance of habitat amount and fragmentation; 2) to compare the accuracy and precision of these different statistical estimates of relative importance under identical conditions using simulated but realistic data; and 3) to make recommendations on the best approaches to use in future studies. In my simulations, I compared 7 statistical methods: standard multiple regression, residual regression, variable or model selection, two multi-model inference measures (sensu Burnham and Anderson 2002), and two variance partitioning approaches.

\section{$2.3 \quad$ Methods}

\section{Literature review}

I searched the literature for studies that compared the effects of habitat amount and fragmentation on an ecological response variable. After excluding patch-scale studies (sensu Fahrig 2003), my review included 33 empirical studies that compared the relative effects of habitat amount and fragmentation, measured at the landscape scale. I reviewed 
the statistical methods used in the studies and the conclusions on which (habitat amount or fragmentation) was more influential.

The studies in my review employed some combination of 6 statistical tools to evaluate the relative importance of fragmentation and amount. These included: 1) residual regression, 2) variable or model selection procedures (stepwise significance or AIC-based selection), 3) averaged coefficients that account for uncertainty in model selection, 4) summed Akaike weights (3 and 4 relate to multi-model inference (MMI); Burnham and Anderson 2002), 5) classical variance partitioning, and 6) hierarchical variance partitioning (HVP, Chevan and Sutherland 1991). I categorized metrics from these methods as either "confidence metrics" (i.e., those that estimate the confidence or uncertainty in a particular predictor's effect, based on either statistical significance or the inclusion of a predictor in a best or final model) or "strength metrics" (i.e., those that estimate the relative strength of a predictor's effect, based on a regression coefficient or an estimate of explained variation).

\section{$2.4 \quad$ Simulations Landscapes variables}

I used 350 real landscapes to provide the landscape variables used in my simulations. The landscapes were $10 \times 10 \mathrm{~km}$ regions of Southern Ontario, Canada; which were clipped from a pre-existing, landcover map of the province with 28 classes and $30 \mathrm{~m}$ cell-resolution (OMNR 1998). I used four landscape variables in my simulations. One variable represented habitat amount ("Amount" = the area of forest cover). Two variables represented habitat fragmentation - habitat edge ("Edge" = length of the edge between forest and all non-forest landcover) and mean patch size ("MnPatch" $=$ average size of forest patches). A fourth variable represented the heterogeneity of 
natural landcover ("Hetero" = number of natural, landcover classes present in the landscape). This fourth variable could be grouped with habitat amount in the broader category of landscape composition variables but in my simulations, it primarily serves to demonstrate the effects of variables that are less strongly correlated $(r \sim 0.5)$. For the first three measures, the original landcover data was reclassified into a binary, forest/nonforest landscape. For the fourth measure (Hetero), the original landcover classification was used. I chose these variables because they are relatively common in landscape ecology studies and they are correlated in these landscapes (Figure 2.1). Landscape variables were measured using the program Fragstats (McGarigal et al. 2002).

In my simulations, Edge acts as a suppressor variable because all predictors are positively correlated (Figure 2.1) but Edge's effects are opposite in sign to all other predictors (i.e., negative). As an example of this suppressor relationship, a regression coefficient for Amount would be effectively zero when $Y=a+2.0 *$ Amount $-2.0 * E d g e+\varepsilon$ is regressed on Amount only, because the effects of Edge on $Y$ suppress those of Amount (and vice versa). However, if $Y$ is regressed on both predictors then the partial regression coefficients are accurate $(|\beta|=2.0)$.

I used both the pair-wise correlation matrix among all predictors as well as each predictor's variance inflation factor (Neter et al 1990) to quantify collinearity. Variance inflation factors (VIF) were calculated for each predictor as the inverse of the coefficient of non-determination $\left(1 /\left(1-R^{2}\right)\right)$ for a regression of that predictor on all others. VIF is a positive value representing the overall correlation of each predictor with all others in a model. Generally, VIF > 10 indicate "severe" collinearity (Neter et al 1990). 
I used real landscapes because I wanted a realistic representation of the covariance structure among predictor variables in empirical studies of habitat loss and fragmentation. I limited my selection of landscapes to 350 that contained $<30 \%$ habitat cover because theoretical studies suggest that fragmentation effects should be most evident in landscapes with low habitat amount and therefore questions of relative importance are most relevant here (Fahrig 1998, Flather and Bevers 2002) and the relationships between many fragmentation metrics and habitat amount are simplified in this range (approximately linear instead of curvilinear). I standardized all predictors to a mean of 0 and a standard deviation of 1.0 so that equal coefficients implied equal effect-strength and to simplify expected variance partitions for each predictor.

\section{Simulated response data}

I generated simulated response data for all 350 landscapes, using a linear equation with coefficients of known value (e.g., $Y=\mathbf{a}+\beta_{1}$ Amount $+\beta_{2}$ Fragmentation $+\varepsilon$ ). The values of these response data could be interpreted as a measure of average abundance of a species within each landscape or any other response with an approximately normal distribution. I used each of the following linear equations to represent 6 true models:

$$
\begin{aligned}
& Y_{a e p h}=a+2.0 * \text { Amount }-2.0 * \text { Edge }+2.0 * \text { MnPatch }+2.0 * \text { Hetero }+\varepsilon \\
& Y_{a e}=a+2.0 * \text { Amount }-2.0 * \text { Edge }+\varepsilon \\
& Y_{a p}=a+2.0 * \text { Amount }+2.0 * \text { MnPatch }+\varepsilon \\
& Y_{a}=a+2.0 * \text { Amount }+\varepsilon \\
& Y_{e}=a-2.0 * \text { Edge }+\varepsilon \\
& Y_{p}=a+2.0 * \text { MnPatch }+\varepsilon
\end{aligned}
$$


The subscripts indicate "influential predictors" - predictors used to create the relevant simulated response (e.g., "e" in $Y_{e}$ refers to Edge). "Uninfluential predictors" are those that were not used to create a particular response (e.g., Edge in $Y_{a p}$ ). The error term (ع) was normally distributed with mean of zero and a variance adjusted so that a correct statistical model (i.e., one that included the same predictors as the true model) explained approximately $50 \%$ of the variation in the response. Response data from each of the 6 true models were replicated 100 times. Among the 100 replications, only the random values of the error $(\varepsilon)$ in the linear equation varied.

\section{Comparing statistical methods}

To compare the different statistical methods, the simulated response data from each of the 6 true models were analyzed using each of the 7 statistical methods of relative importance (i.e., partial coefficients from a multiple regression, plus the methods identified in my literature review). I assessed the accuracy (in comparison to the true value) and variation across the 100 replications in the estimates of relative importance from each statistical method (i.e., the mean and variation in the; partial coefficients, relative proportion of variance explained, or relative importance according to the summed Akaike weight). Finally, each of the 7 statistical methods were compared under each of 3 conditions, by varying which predictors were included in the statistical analyses for each true model: 1) correct predictors - the analysis contained all of the influential predictors and no uninfluential predictors; 2) too many predictors - the analysis contained all of the influential predictors as well as some uninfluential predictors; and 3) too few predictors the analysis contained only a subset of the influential predictors. The entire simulation experiment consisted of 7 statistical methods applied under 3 different conditions to 
simulated data created under 6 true models where the data creation was replicated 100 times.

All simulations were conducted using R statistical software (www.r-project.org) with additional packages for some of the specific statistical analyses including "hier.part" for HVP and "MuMIn" for MMI calculations across all possible sub-models (available online at: http://r-forge.r-project.org/projects/mumin/).

\section{$2.5 \quad$ Results}

A majority of the studies that I reviewed found that habitat amount was generally more important than fragmentation in determining the effects of landscape structure on biodiversity (Table 2.1). There were no clear trends in published results that would suggest particular methods were more likely than others to find amount or fragmentation most important. Additional results of the literature review are in the following, methodspecific sections.

\section{Partial coefficients from a multiple regression}

This approach was not used on its own in any of the reviewed studies, yet I found that multiple regression coefficients were unbiased estimates of effect strength when the correct predictors were available (Figure 2.2 A) and also when too many predictors were available (Figure 2.2 D). However, as for all methods, coefficients were biased if too few predictors were available. If the missing predictor was not a suppressor, then the absolute values of the coefficients were over-estimated (see Amount in Figure 2.3 A). If the missing predictor was a suppressor, then the absolute values of the coefficients of the included predictors were underestimated (Figure $2.3 \mathrm{~B}$ ). In both cases, the coefficients were biased to a degree dependent on their correlation with the missing predictor. 


\section{Residual regression}

Residual regression was used in 10 of the 33 studies I reviewed (Table 2.1). All 10 used residuals for the fragmentation variables, leaving the habitat amount variables intact and were therefore supposedly biased towards the effects of habitat amount. However, this bias was not evident in the results. Indeed, only $50 \%$ of the residual regression studies, compared to approximately $70 \%$ of the remaining studies, concluded that amount was more important than fragmentation (Table 2.1).

In my simulations, if the amount and fragmentation predictors had similar effect directions and were positively correlated then residual regression overestimated the importance of the intact predictor and underestimated the importance of the residual predictor (Figure 2.4 B) as suggested by Koper et al (2007). However, if the two predictors had a suppressor relationship (e.g., Amount and Edge), the importance of both predictors was underestimated (Figure 2.4 A). In addition, with a suppressor relationship, the presumed bias in residual regression was actually reversed because the residual predictor's coefficient was less underestimated than the intact predictor's (i.e., the residual predictor was more likely to be significant and had a larger coefficient value, Figure 2.4 A).

\section{Variable/model selection}

Fourteen of the studies in my review used some sort of stepwise selection procedure to identify important variables for inclusion in a final or best model (Table 2.1). Some (but not all) studies took steps to limit correlations among predictors to avoid potentially spurious models (Kruskal and Majors 1989, Clark and Troskie 2006). This was generally done by removing one of a pair of correlated predictors from the model (either during the stepwise selection or before the analysis by removing one of each pair 
of highly correlated predictors). No studies provided estimates of potential effect strength for any of the removed predictors.

In my simulations, I used AIC to choose the best model from among all possible combinations of a global model (i.e., a model that includes all of the available predictors). When the correct predictors were available, this approach almost always selected the global model (i.e., the correct model, Figure $2.2 \mathrm{~B}$ ). When too many predictors were available, the best model included uninfluential predictors at least $14 \%$ of the time (Figure 2.2 E). However, this inclusion of uninfluential predictors did not strongly bias the regression coefficients of the influential variables. If too few predictors were available, the influential predictors were retained in the best model but the coefficients were biased in the same way as partial coefficients from a multiple regression (Figure 2.3).

\section{Weighted mean coefficients}

Ten of the studies I reviewed used coefficients averaged across models to estimate relative importance (Table 2.1). In MMI, these averaged coefficients are a strength metric for each predictor, which is calculated as the weighted mean partial regression coefficient, averaged across all supported models (i.e., models for which Akaike weight $>$ 0.05 , or $\Delta \mathrm{AIC}<4$ ) and weighted by the Akaike weight (an estimate of the support for each model in the data, Burnham and Anderson 2002). In my simulations, when the influential predictors were available (i.e., the correct predictors or too many predictors), this method generated unbiased estimates but the variance around the estimates was slightly higher than for other coefficient-based approaches (compare Figures $2.2 \mathrm{C}$ and F). When too few predictors were available, the coefficients were biased in the same way 
and to the same degree as for the model selection and multiple regression approaches (results similar to those in Figure 2.3).

\section{Summed Akaike weights}

The same ten studies that used MMI averaged coefficients, also included a confidence metric for each predictor, which was calculated as the sum of the Akaike weights for all supported models that include each predictor ("variable importance" sensu Burnham and Anderson 2002). With this metric, if there are numerous competing models for which there is some support, then variables that are included in models with higher weights and/or included in more of the supported models will be ranked higher in relative importance. This measure is biased towards predictors that are included in more candidate models (Burnham and Anderson 2002, pg 169) but I found no acknowledgement of this potential bias in the reviewed studies.

In my simulations, the summed Akaike weight was a liberal confidence metric, in that uninfluential predictors were always allocated some importance. Although the average estimate was generally lower for uninfluential predictors, there was much variation and in some replications uninfluential predictors received very high importance scores (Figure 2.5 B). In addition, this metric underestimated the importance of influential predictors that were more highly correlated (e.g., Amount and Edge in Figure $2.5 \mathrm{~A})$.

\section{Classical Variance Partitioning}

Five of the reviewed studies partitioned the variation in the response into portions that were explained independently by each predictor and portions that were shared among predictors (Legendre and Legendre 1998, pg 528). These studies used this approach to estimate effect strength and importance of predictors in a full model, by comparing the 
size of the independently explained portions. However, when the predictors are highly correlated, then the independent portions represent only a fraction of the predictor's true effect or importance.

In my simulations, this approach could generally distinguish between influential and uninfluential predictors but the ranking of influential predictors was biased. Uninfluential predictors did not, on average, explain any of the variation independently (Figure $2.6 \mathrm{~A}$ ). In contrast, even highly correlated influential predictors were always allocated a non-zero share. However, the ranking of influential predictors was highly dependent on the correlations among predictors. When all four predictors contributed an equal share $(25 \%)$ to the response (i.e., $\left.Y_{a e p h}\right)$ the average across all 100 replications of the independently explained variation for each predictor ranged from $4 \%$ to $21 \%$ (Figure $2.6 \mathrm{~A})$.

As for all methods, this approach was also biased when too few predictors were available but the type of bias depended on suppressor effects. The importance of influential predictors was underestimated in all situations (Figure $2.6 \mathrm{~A}, \mathrm{~B}$, and D) except when a substantial, non-suppressor variable was missing from the analysis (Figure 2.6 C). In that case, the importance of the modeled predictor with similar qualitative effects as the missing predictor was overestimated because it was allocated the explanatory power of the missing predictor while the modeled predictor with different qualitative effects (suppressor relationship) was not. In contrast, when the missing predictor had a suppressor relationship with both retained predictors neither took on its explanatory power; instead, the missing predictor's effect reduced the variation explained by all retained predictors (Figure 2.6 D). 


\section{Hierarchical Variance Partitioning}

The HVP approach was applied in 4 of the 33 studies (Table 2.1). HVP decomposes all of the variation explained by the full model (including the shared component) into components that can be allocated to individual predictors (Chevan and Sutherland 1991). HVP partitions the shared components of the full model by assuming that shared component within each hierarchical level of all possible sub-models can be divided equally among correlated predictors. HVP is one of a number of closely related metrics of relative importance that are more commonly applied in the social sciences (Kruskal 1987, Gromping 2007).

In my simulations, the strength estimates from HVP analyses were strongly affected by both the predictor's correlation with other predictors and the direction of its effect. When all predictors had equal absolute effects on the response (i.e., $Y_{a e p h}$, Figure 2.6 E), importance was overestimated for those that were less correlated and had positive effects (i.e., MnPatch and Hetero) and underestimated for those that were more correlated and had positive effects (i.e., Amount). Importance of the suppressor variable Edge was greatly underestimated. HVP always allocated some of the explained variation to every predictor in the model, even uninfluential ones (Figure 2.6 F). The importance was consistently overestimated for the following three groups: uninfluential predictors, predictors that were less correlated than the others (lower VIF), and all predictors when influential, non-suppressor predictors were missing from the analysis

\subsection{Discussion}

\section{Comparing methods}

The main finding of my simulation experiment was that standard multiple regression performed as well or better than all of the methods that have been used to 
account for collinearity. Standardized partial regression coefficients within a reasonably well specified model are useful measures of effect strength even for correlated predictors. Within the range of true models and conditions that I simulated, standardized partial regression coefficients of highly correlated predictors were unbiased whether from a straight-forward multiple regression, the best model identified by AIC, or averaged across all supported models using MMI in an information theoretic framework - although the MMI approach slightly increased the variance around the estimate (Figure 2.2). Perhaps the strong performance of multiple regression should not come as a surprise since there is a long history of using regression to control for potentially confounding effects and continuous predictors are almost always correlated to some degree (Legendre and Legendre 1998). However, considering the added complexity from many statistical manipulations intended to remove collinearity or the alternative methods intended to circumvent the issue, it is remarkable that these efforts may be worse than doing nothing at all.

Even though collinearity causes high variance around partial regression coefficient estimates and reduced statistical power (Neter et al 1990; Graham 2003), my simulations suggest that the alternatives to multiple regression have similar or even worse problems. The summed Akaike weights of highly correlated predictors were underestimated and more highly variable than for less correlated predictors (Figure 2.5). In addition, the estimates from variance partitioning approaches and residual regression were both highly variable and biased (Figures 2.4 and 2.6). By contrast, although more correlated predictors were more likely to be found erroneously insignificant (i.e., increased Type II error - reduced statistical power), in the context of my simulations, multiple regression 
identified even the most highly correlated influential predictors as statistically significant in $>90 \%$ of the replications (Figure 2.2).

Ecologists have been trained to remove collinearity from ecological regression models, in part, because of suggestions that collinearity causes "biased" coefficient estimates (Petraitis et al 1996, Graham 2003). However, in my simulations partial regression coefficients were unbiased for influential predictors, even when the predictors were highly correlated. The difference between my results and the results of these previous studies is entirely due to interpretation. Interpreting partial coefficients as "biased" assumes that the simple or univariate coefficient of each predictor with the response represents the "truth". In fact, if the two correlated predictors have additive functional relationships with the response (e.g., distinct ecological processes such as population size and negative edge effects), then the partial coefficients are the best representation of the true effects and the univariate coefficients are the "biased" estimates. I only observed bias in the partial regression coefficients when an important confounding effect was missing from the model; and indeed, this was true for all methods. Controlling for confounding effects requires knowledge of the relevant ecological processes in the system being studied and clear links between those processes and the predictor variables in the statistical model.

Residual regression studies are known to have predictable bias (Koper et al. 2007) but my results show they may also be biased in unexpected ways. Residual regression is biased towards the intact variable (usually habitat amount) if the effects of amount and fragmentation do not conflict (i.e., conditions that lead to box B in Figure 2.7 and similar to the bias shown in Koper et al 2007). However, effect strength and statistical 
significance in published studies may have been underestimated for both amount and fragmentation if the variables were suppressors (i.e., conditions that lead to box A in Figure 2.7). Indeed, some published estimates may even have been biased in favor of the residual variable (usually fragmentation), because my simulations suggest the residual variable's effect is less underestimated than the intact variable. Therefore, even if a researcher had wished to impose an initial priority on one of the variables, residual regression may have given inaccurate results. Suppressor effects may explain why the results of studies that used residual regression did not reflect the presumed bias of habitat amount over fragmentation (Table 2.1). My results cast serious doubt on previous research that applied residuals to distinguish between the effects of habitat fragmentation and amount.

Partitions of independently explained variation should be avoided as measures of the relative effects of amount and fragmentation because they reveal as much about the correlation structure among predictors as their relative effects. When true effects were identical across predictors, the classical independent variance partitions ranked the predictors according to their level of correlation with the other predictors. The independent partitions were approximately what would be expected considering the correlation structure of the predictors but they did not accurately represent the true independent contributions of each predictor. Similarly, estimates of relative importance using HVP depended on how correlated a predictor was but were further biased by the direction of a predictor's effect (i.e., underestimated for highly correlated predictors and suppressor variables and overestimated for others). The flaw in using partitions of independently explained variation as estimates of importance is that they combine 
estimates of effect strength and confidence. It is not clear from an independent variance partition (classical or HVP) whether a variable has a strong but uncertain effect or a certain but weak effect. In a management context, this distinction is clearly important. Less confounded metrics of effect strength (such as coefficient-based estimates) presented in combination with confidence estimates (such as summed Akaike weights or the coefficient's confidence intervals) will be more informative than estimates of explained variation.

\section{Toward a synthesis}

Future studies of the relative importance of amount and fragmentation should explicitly report both the variance inflation factor for each predictor and the pair-wise correlations among all predictors. Without this information, I could not synthesize the literature in my review while accounting for the biases identified here. Many studies reported only a maximum, pair-wise correlation coefficient (e.g., $\mathrm{r}<0.8$ ), or removed predictors that were "highly" correlated with another predictor in the analysis using an arbitrary cut-off value for pair-wise correlations (usually $r>0.5-0.7$ ). These simple criteria give no information on the remaining correlations, which may still influence results. Indeed, considering only pair-wise correlations may not actually reduce the statistical problems caused by collinearity. Complicated relationships among predictors can result in large increases in error around coefficient estimates for predictors that nonetheless have very low pair-wise correlations with all other predictors (Neter et al 1990).

I did not find a single reference to potentially conflicting effects of correlated predictors (suppressor effects) in any of the reviewed studies. However, suppressor effects have very likely influenced the results of studies that used residual regression or 
HVP; they may also have led to underestimates of effect strength for retained variables in any of the methods, if one of a pair of suppressor variables was removed from the analysis because it was "highly correlated". In an ecological context, suppressor variables may represent important effects. For example, increasing habitat amount may have a positive effect on a population through an increase in population size but the concurrent increase in edge length may have a negative effect that acts through an unrelated mechanism (e.g., increases in mortality rates from predators that use habitat edges). In conditions of conflicting ecological processes, using residual regression, HVP, or arbitrarily removing one of a pair of highly correlated variables from a regression model, will give biased estimates of relative importance.

\section{Confronting collinearity}

There are two considerations that should inform decisions on including particular variables in future studies of the relative effects of habitat amount and fragmentation. First, if two predictors are thought to represent distinct ecological processes or mechanisms then both should be included in the analysis. An understanding of the important ecological processes and mechanisms is essential if statistical modeling is to advance ecological understanding (MacNally 2000). Removing variables simply because they are highly correlated with each other or have a weak univariate correlation with the response can lead to uncontrolled, confounding effects that decrease the explanatory power of the final model and generate biased measures of effect strength. Second, the direction of the effect and the correlation between two variables must be considered. If two predictors have a suppressor relationship (i.e., opposite qualitative effects and a positive correlation or similar qualitative effects and a negative correlation), removing one will underestimate the effects of the remaining predictor. If two predictors do not 
have a suppressor relationship (i.e., similar qualitative effects and a positive correlation or opposite qualitative effects and a negative correlation), removing one will overestimate the effects of the other. If these two considerations are followed, standardized partial regression coefficients will be unbiased estimates of the relative importance of amount and fragmentation, even when predictors are highly correlated. 


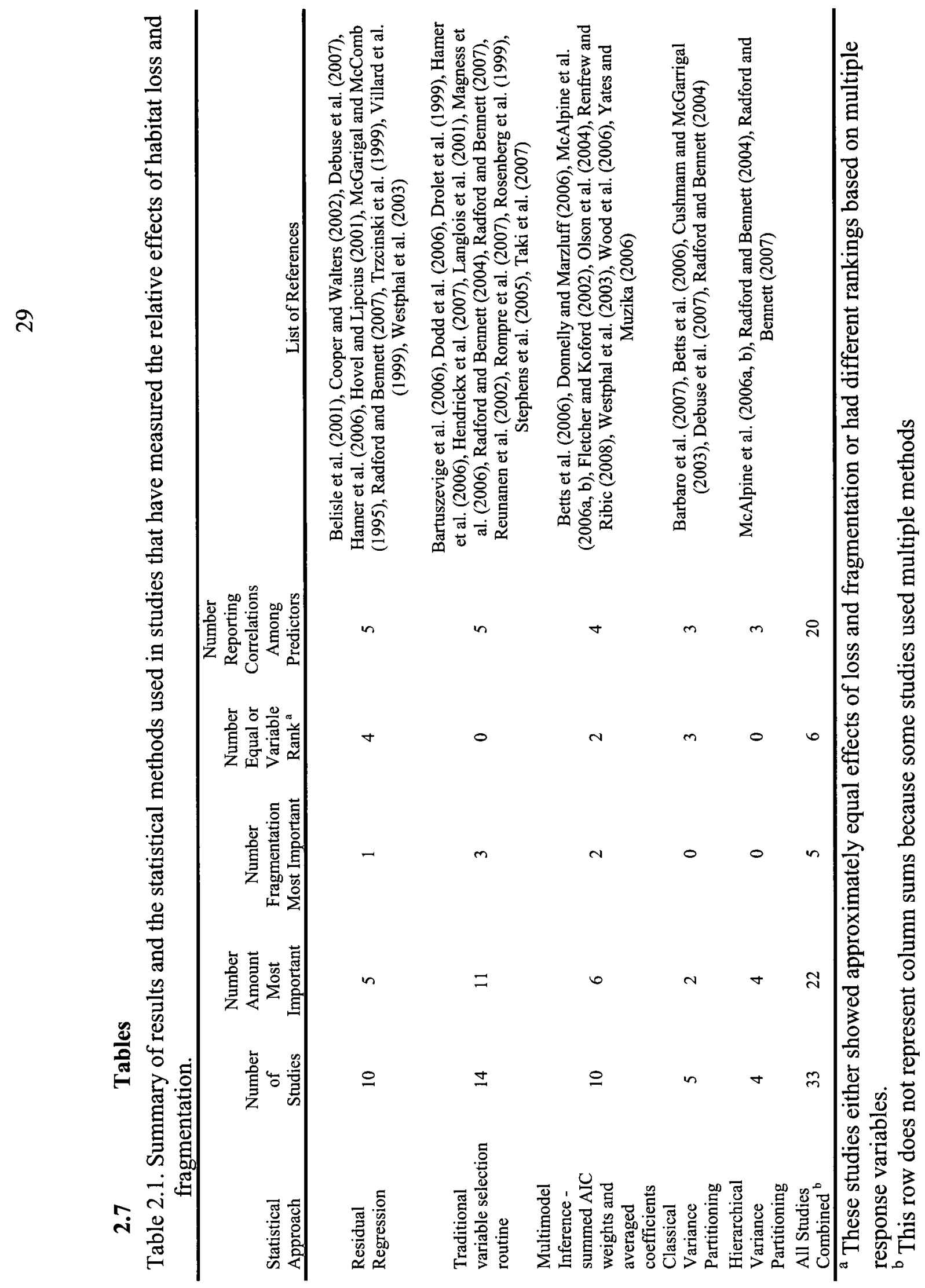



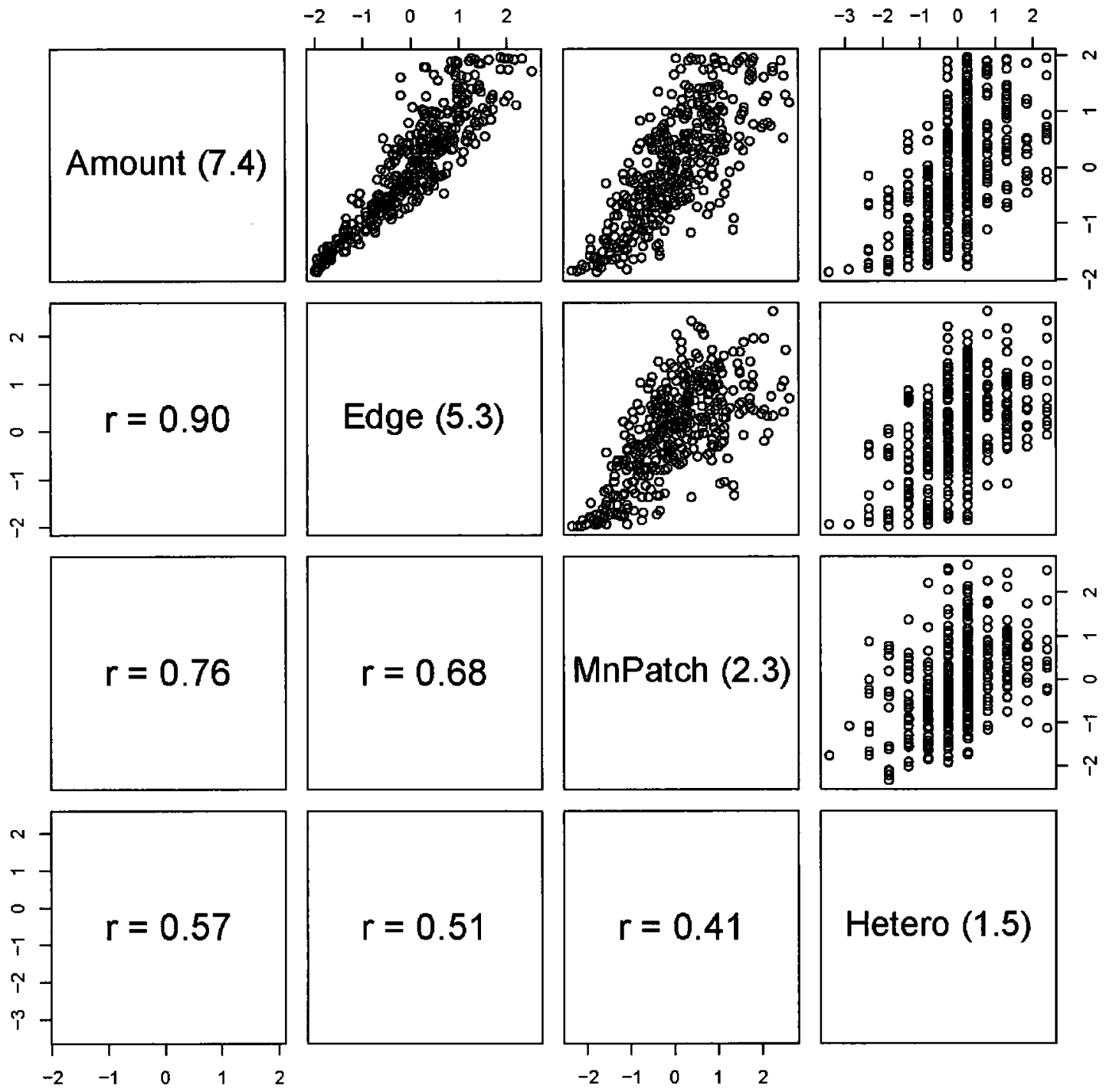

Figure 2.1. Correlation matrix of standardized landscape predictor variables $(\mathrm{n}=350)$. Numbers in parentheses are the variance inflation factors for each predictor, and numbers below the diagonal are Pearson correlation coefficients for all predictor pairs. 


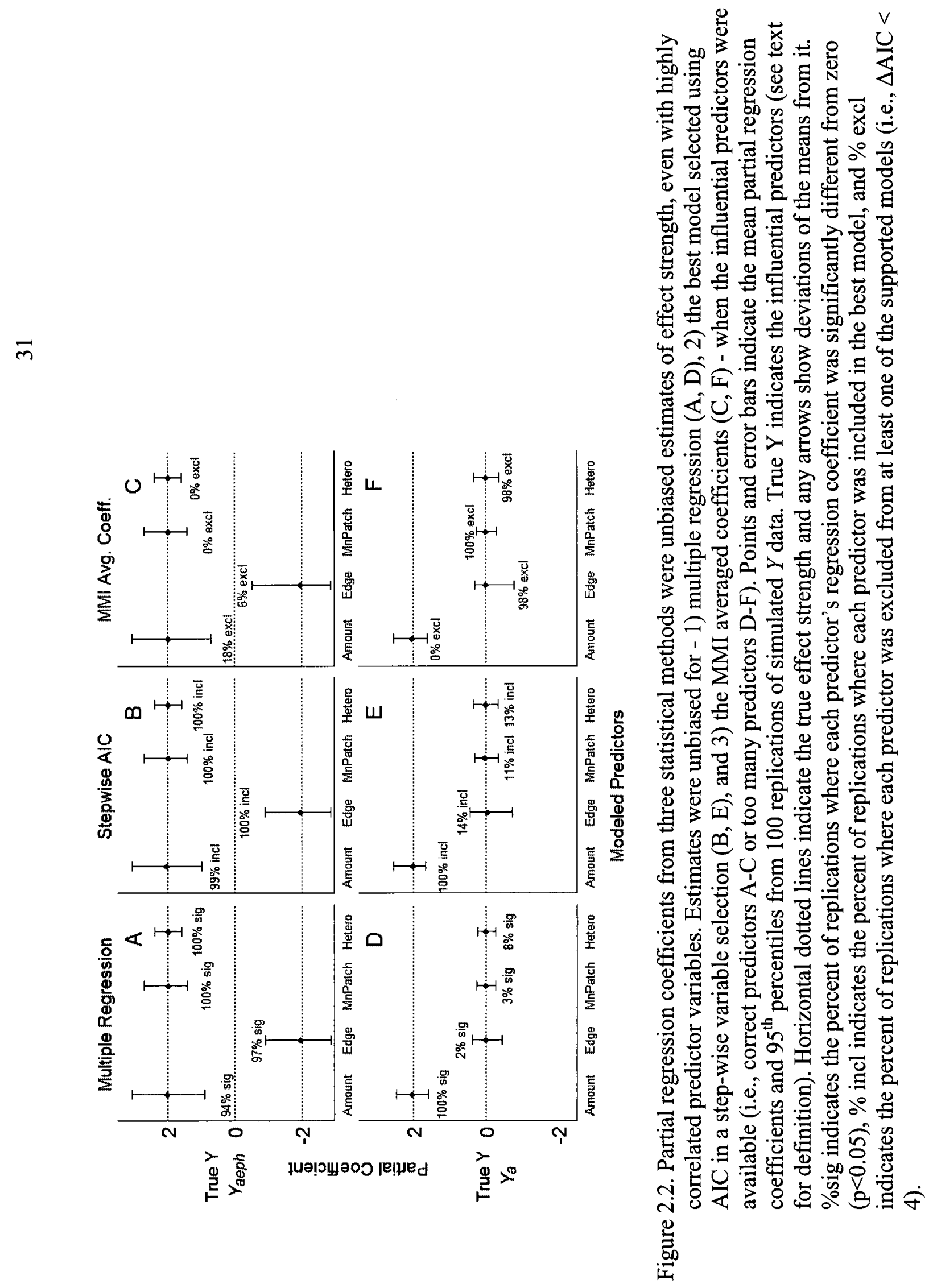




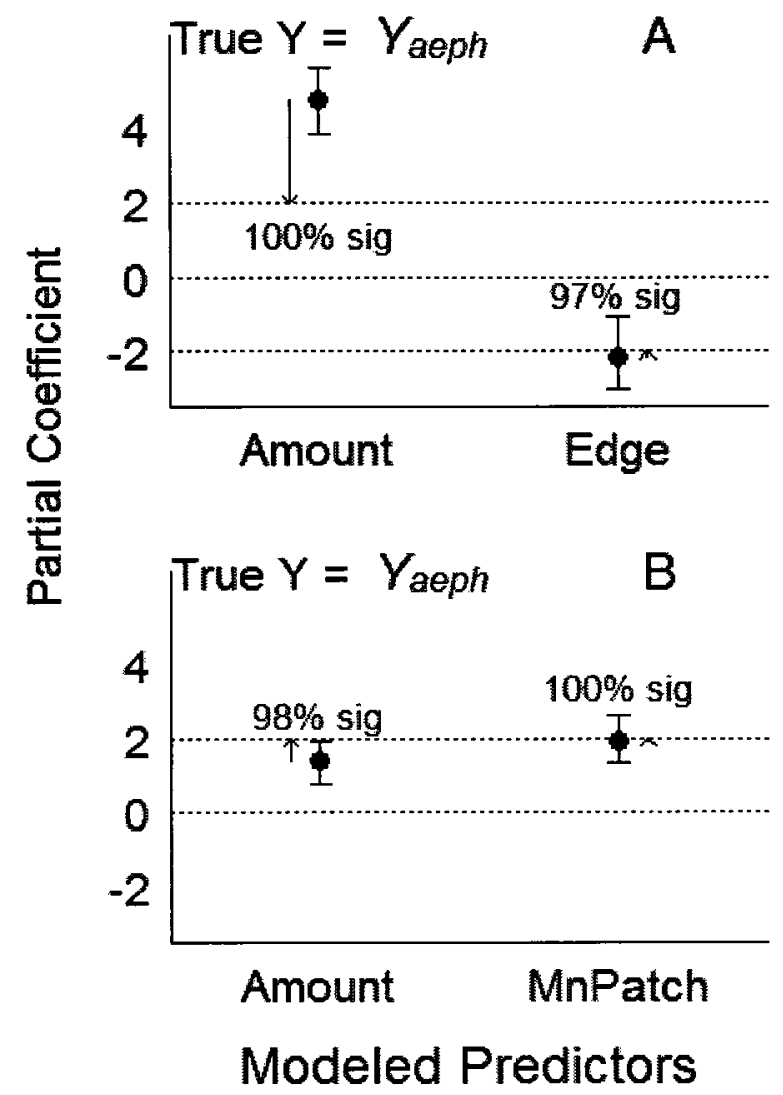

Figure 2.3. Partial regression coefficients were biased if influential predictors were missing from the model and the strength and direction of bias depended on suppressor relationships. In A, the missing influential predictors were nonsuppressors and therefore the most highly correlated predictor (Amount) was overestimated. In B, one of the missing influential predictors (Edge) was a suppressor and therefore the most highly correlated predictor (Amount) was underestimated. Partial regression coefficients from a multiple regression are shown here but the same bias applied to all three coefficient-based estimates included in Figure 2.2. See Figure 2.2 for explanation of text and symbols in the plot. 


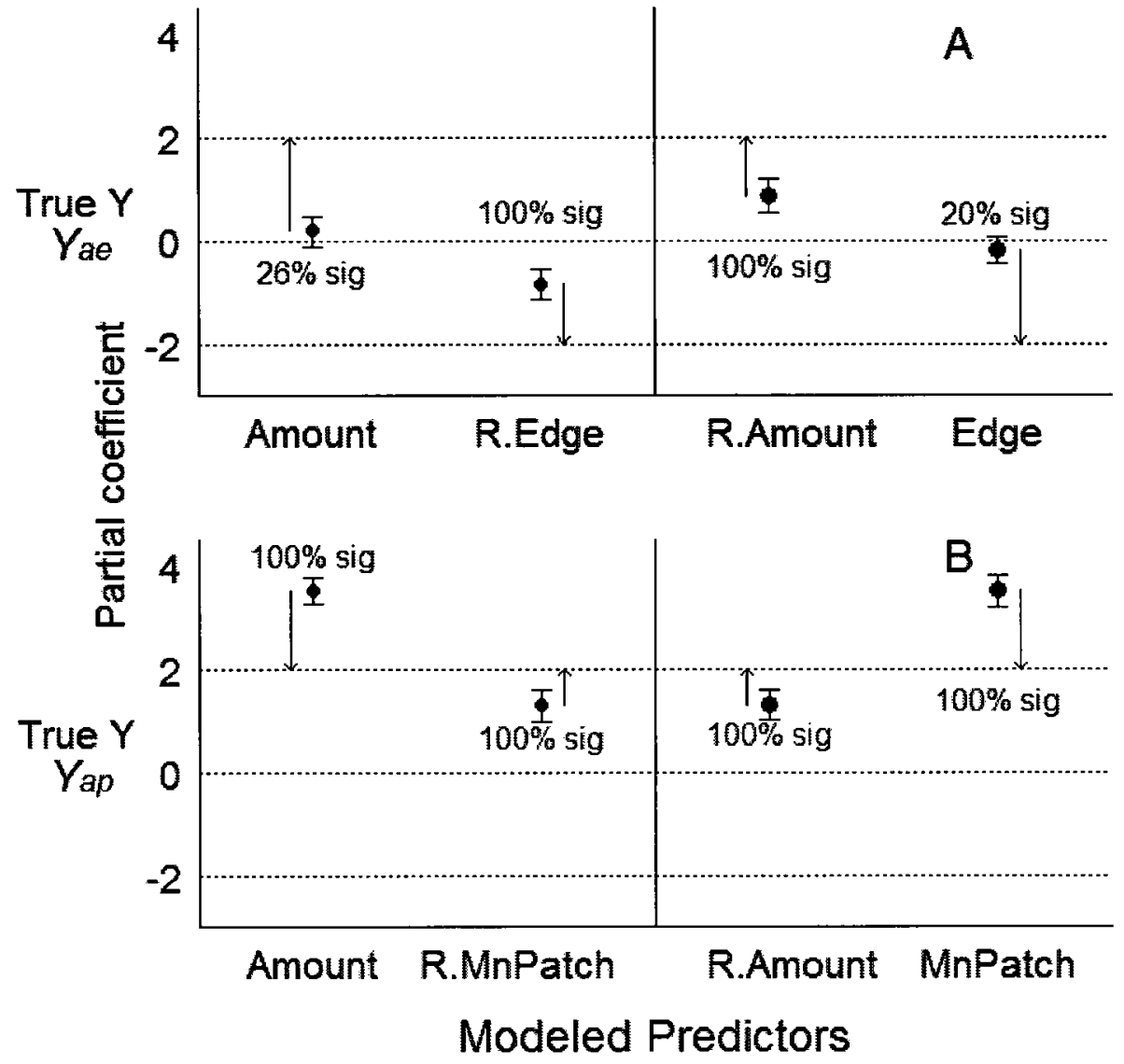

Figure 2.4. Residual regression is biased but the bias depends on suppressor effects. With suppressor effects (A), both amount and fragmentation effects are underestimated and the analysis is biased towards the residual predictor whether fragmentation (left side of A) or amount (right side of A). When there is no suppressor relationship (B) the analysis is biased towards the intact predictor. The prefix "R." indicates the residual predictor in each of the 4 separate analyses. See Figure 2.2 for explanation of text and symbols. 


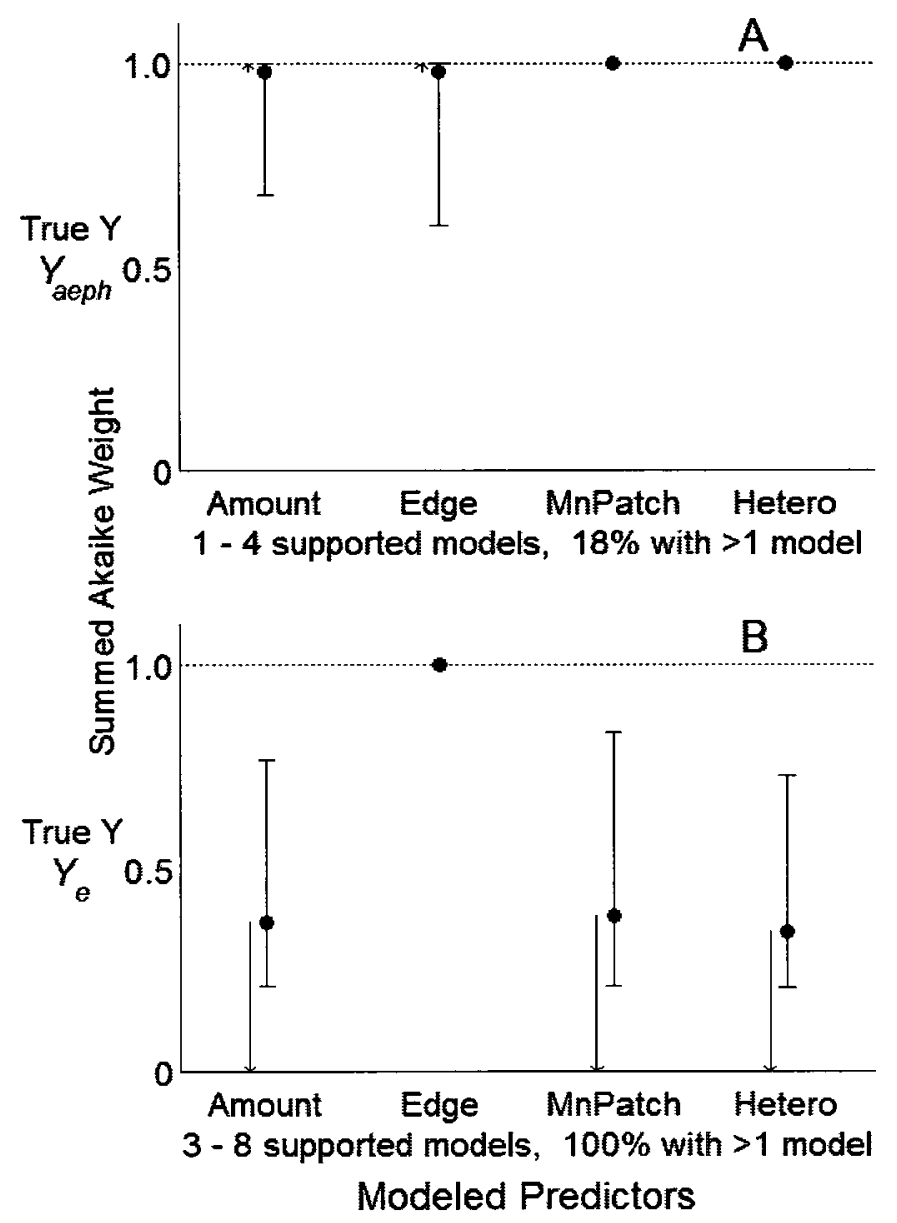

Figure 2.5. Summed Akaike weights overestimated the importance of uninfluential predictors (B) and in some replications, underestimated the importance of influential predictors that were more highly correlated (A). Text below each figure indicates range in the number of supported models (i.e., $\Delta$ AIC $<4$ ) across 100 replications and the percent of replications for which more than 1 model was supported. Points and error bars indicate the mean summed Akaike weights and $95^{\text {th }}$ percentiles from 100 replications of simulated $Y$ data. See Figure 2.2 for explanation of other text and symbols. 
$n$

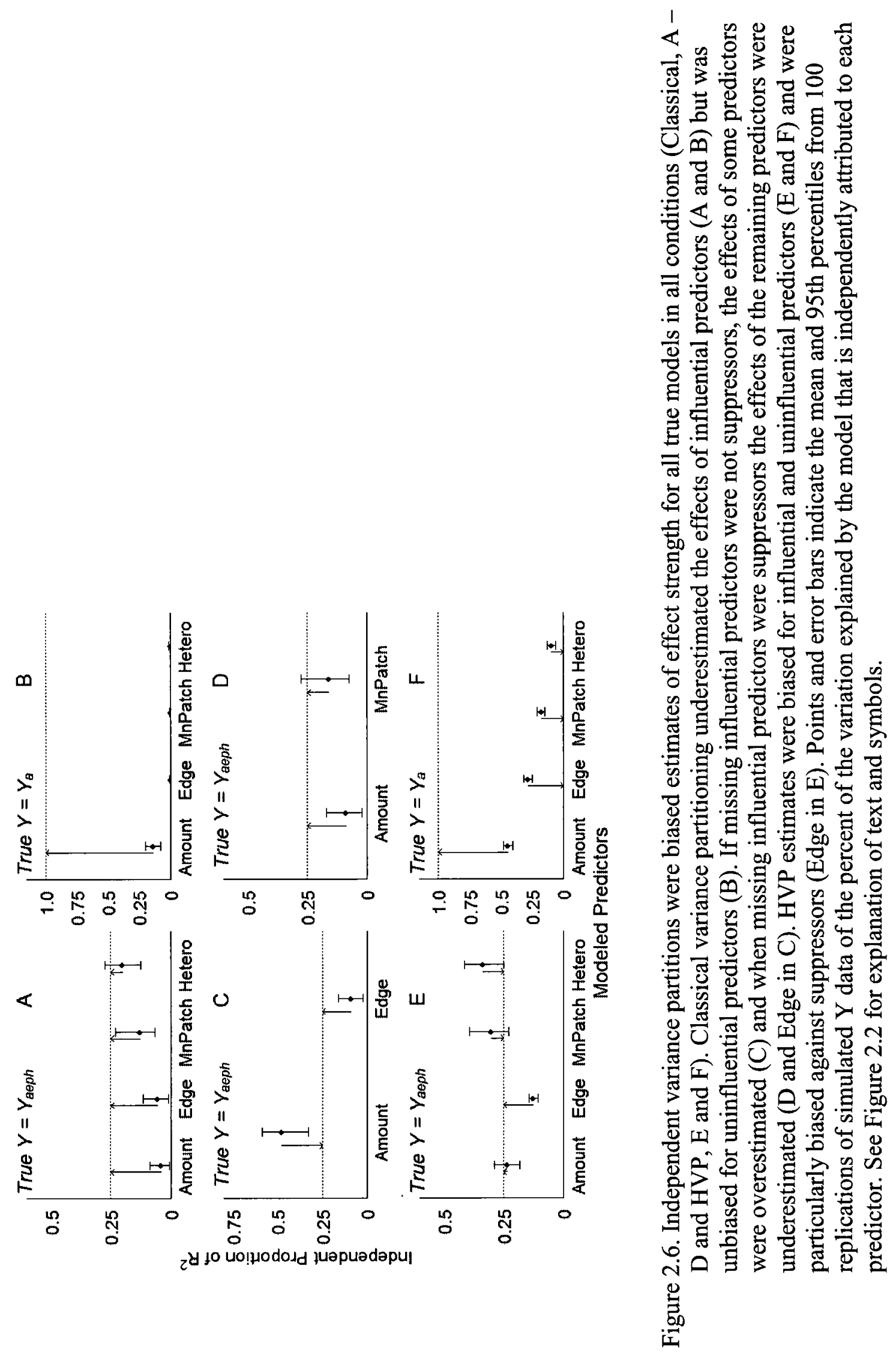




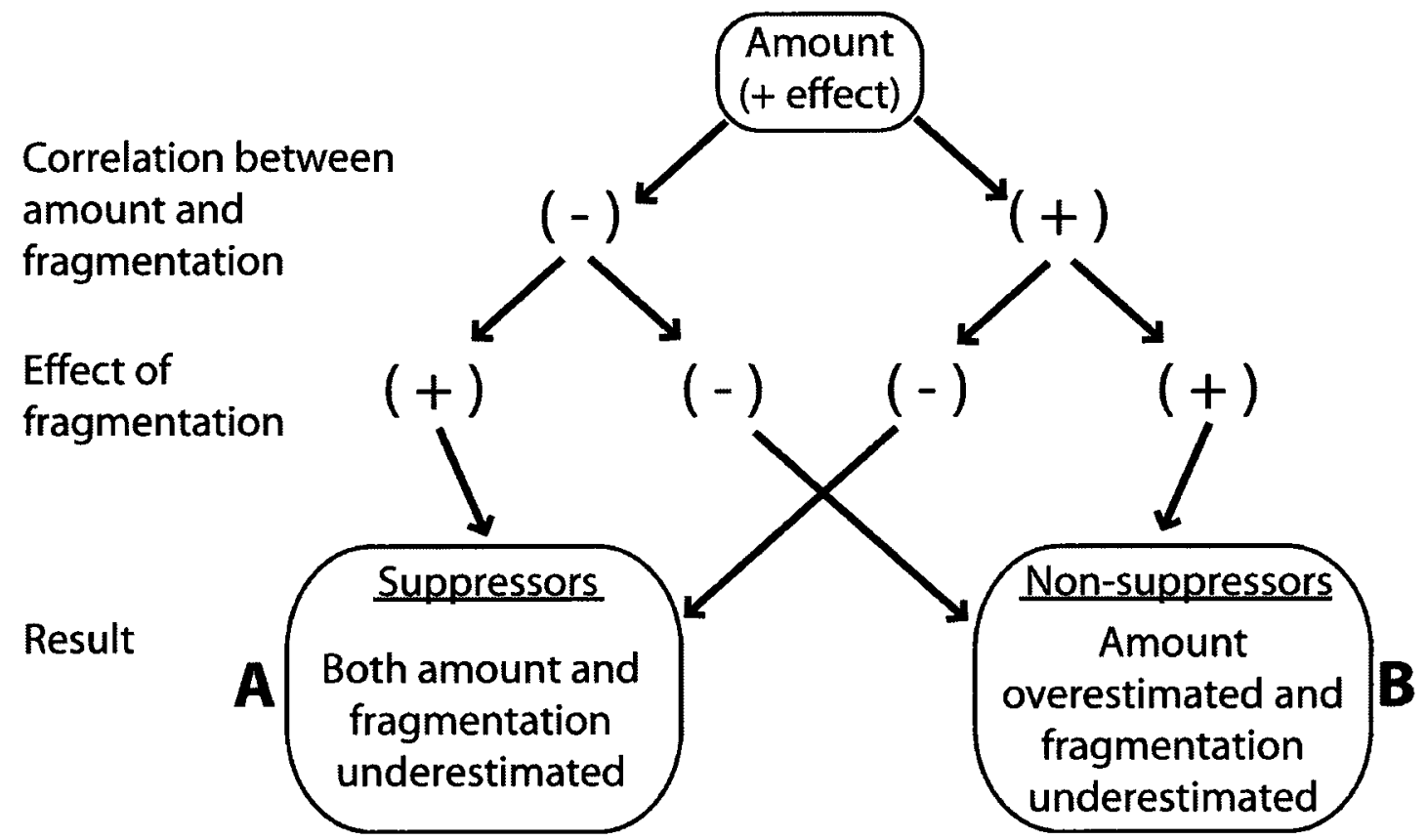

Figure 2.7. The bias in residual regression depends on suppressor effects (i.e., the correlation between amount and fragmentation and whether the fragmentation measure has a positive or negative effect on the response). This figure shows the qualitative bias in published studies that used residual regression to create fragmentation metrics orthogonal to habitat amount. The strength of the bias is directly related to the magnitude of correlation between the original predictors in the published study. If habitat loss was measured instead of amount a negative effect of loss on the response would be expected. In this case, switching the contents of boxes A and B would show the resulting bias. 
Chapter 3. Landscape size affects the relative importance of habitat amount, habitat fragmentation, and matrix quality on forest birds.

(Adapted from: Smith A.C., C. M. Francis, and L. Fahrig. In Press. Landscape size affects the relative importance of habitat amount, habitat fragmentation, and matrix quality on forest birds. Ecography)

\subsection{Chapter Summary:}

It is important to understand the relative effects of landscape habitat loss, habitat fragmentation, and matrix quality on biodiversity, so that potential management options can be appropriately ranked. However, their effects and relative importance may change with the size of the landscape considered because the multiple (and potentially conflicting) ecological processes that are influenced by landscape structure occur at different spatial scales (e.g., dispersal, predation, foraging). I estimated the relative effects of habitat loss, habitat fragmentation, and matrix quality (measured as the amount of forest, the proportion of forest area contained in large core forests, and the density of roads respectively) on fragmentation-sensitive forest birds in Southern Ontario, Canada using a range of landscape sizes $\left(0.8 \mathrm{~km}^{2}-310 \mathrm{~km}^{2}\right)$. I used three complementary statistical approaches to estimate relative effects of these correlated landscape factors -1 ) multiple regression, 2) information theoretic (AIC) estimates of the most parsimonious model, and 3) multi-model inference to average effects across all supported models. I controlled for spatial autocorrelation, local habitat, roadside sampling bias, time of day, season, habitat heterogeneity, and the interaction between the effects of habitat amount and fragmentation. I found that relative effects of habitat amount and fragmentation were scale dependent; habitat amount had a consistently positive effect that was consistent over more than two orders of magnitude in landscape area $\left(\sim 1 \mathrm{~km}^{2}-300 \mathrm{~km}^{2}\right)$. In contrast, the effects of habitat fragmentation depended on the size of the landscape 
considered. Indeed, for Veery (Catharus fuscescens), habitat fragmentation had positive effects at one scale and negative effects at another. The effects of matrix quality were generally weak and changed little with scale. For the number of fragmentation sensitive species and the presence of Veery, habitat amount was most important in large landscapes and habitat fragmentation in small landscapes but for the presence of Ovenbird (Seiurus aurocapilla), habitat amount was most important at all scales.

\subsection{Introduction}

The amount of habitat in a landscape affects biodiversity through multiple processes including reproduction, mortality, dispersal, local extinction, and species interactions operating at various spatial and temporal scales (Addicott et al. 1987). Modelling and empirical studies (Rosenzweig 1995; Fahrig 2003; Ewers and Didham 2006) have shown that the effects of habitat amount on biodiversity are consistently positive and strong across regions, habitats, and taxa.

In addition to the direct effects of habitat loss, habitat fragmentation per se (i.e., breaking apart of habitat, independent of habitat amount) may affect biodiversity, particularly in regions with less than 20-30\% habitat cover (Andren 1994; Fahrig 1998). In empirical studies, the effects of habitat fragmentation per se (hereafter fragmentation) are about as likely to be positive as negative (Fahrig 2003). Negative effects may arise from a reduction in the proportion of patches that are large enough to support stable or productive (source) populations (e.g., Nol et al. 2005), or from negative edge effects (Chalfoun et al. 2002). Positive effects of fragmentation may arise through a reduced probability of simultaneous extinctions of local populations (den Boer 1981), reduced inter-patch distances (Fahrig 2003), increased landscape complementation (access to 
resources in the matrix, Dunning et al. 1992, Law and Dickman 1998), or increased immigration rate (Bowman et al. 2002)

Beyond the amount and fragmentation of habitat in the landscape, the quality of the intervening space (the matrix) can also strongly affect biodiversity (Dunford \& Freemark 2005). Matrix quality can influence species' abundances and distributions in suitable habitat through several processes (Kupfer et al. 2006): mortality during dispersal (Fahrig 2002); changes in movement, which in turn affect patch colonization (Ricketts 2001); influences on microclimate within habitat patches (Burke \& Nol 1998); and changes in predators and parasites within the matrix that influence survival and reproductive success within habitat patches (Chalfoun et al. 2002; Porneluzi and Faaborg 1999).

Habitat amount, habitat fragmentation and matrix quality may all affect biodiversity but the relative effects of these three landscape factors may be scaledependent (Fahrig 1998; Krawchuck and Taylor 2003). Processes such as reproduction, dispersal success, mortality in the matrix, foraging, nest predation, and nest parasitism occur at different rates and within different ecological neighbourhoods (Addicott et al. 1987). Therefore, the effects of these processes should be apparent at different spatial scales. If fragmentation has positive effects on some of these processes and negative effects on others, then the net effect of fragmentation may be scale dependent. For species richness, the net effect of fragmentation on multiple processes may also depend on scale if different species respond at different scales. In contrast, the effects of habitat amount should be consistently positive because although habitat amount also represents multiple ecological processes, these processes all have positive effects. Similar to habitat 
amount, increasing matrix quality should have exclusively positive effects and should therefore be less scale dependent than fragmentation.

Assessing how the relative effects of landscape factors change with scale is vital to inform conservation decisions. Habitat amount, fragmentation, and matrix quality may be manipulated independently of one another to some degree (e.g., changes in fragmentation or matrix quality, for a given amount of habitat). Therefore, assessing the relative importance of these landscape factors allows the ranking of alternative management options by their relative potential benefits to biodiversity (Sutherland et al. 2004). However, if the relative effects are scale-dependent, then the relative ranking of potential management actions will depend on the spatial jurisdiction of the managing body (e.g., landowner, municipality, regional, or national organization) and the spatial extent of a management plan.

In this study I asked the question: Do the effects of habitat amount, habitat fragmentation, and matrix quality on fragmentation-sensitive forest birds depend on the size of the landscape considered (i.e., are they scale-dependent)? I predicted that the effects of habitat amount and matrix quality would be consistently positive across all scales and that the effects of habitat fragmentation would be more scale-dependent.

\subsection{Methods}

\section{Study Area}

The study area was the $201,000 \mathrm{~km}^{2}$ portion of North American Bird Conservation Region 13 that is located in Ontario, Canada (hereafter, BCR 13, Figure 3.1). The study area is entirely within the Mixedwood Plains ecozone (Ecological Stratification Working Group 1996). This area is heavily affected by human activity, is dominated by agriculture and includes the country's largest urban centre. Forested, agricultural, and urbanized 
areas account for approximately $27 \%, 60 \%$, and $8 \%$ of the landcover in the region, respectively. Agricultural activities in the region are diverse, ranging from low intensity pasture, and hay production to high intensity row crops such as soy and corn. Most of the larger, continuous areas of forest are near the northern boundary of the study area and to the south, near the shores of the Great Lakes. Forests are primarily mixed deciduousconiferous throughout the study area; coniferous species dominate in the more northern parts while deciduous species dominate in the south.

\section{Predictor Variables}

I measured 3 primary predictor variables using ArcGIS 9.2 (ESRI, Redlands California), in landscapes of five different radii - $0.5,1.0,2.0,5.0$, and $10.0 \mathrm{~km}-$ centered on the plots where bird surveys were conducted. All predictors were standardized to a mean of 0 and a standard deviation of 1 to allow comparison of regression coefficients. The three predictor variables were: 1) habitat amount (Forest) - the percentage of the landscape area that was forested; 2) core forest (Core) - the percent of forest cover that is $>100 \mathrm{~m}$ from a non-forest edge and $>90$ ha in total size (i.e., a subset of the area within Forest that represents large, compact forest patches, which are thought to sustain source populations of these fragmentation sensitive species, Nol et al. 2005); and 3) road density (Road) - the summed length per unit area of all paved roads within the landscape $\left(\mathrm{km} / \mathrm{km}^{2}\right), \log$ transformed so that it was normally distributed. Road data were from the 2005, Canadian National Topographic Data Base. These variables were chosen to represent the three key landscape factors; 1) habitat amount (Forest), 2) habitat fragmentation (Core), and 3) matrix quality (Road).

I used a binary classification of habitat/non-habitat, which combined all forested landcover types into a single habitat class. For each species, this definition of habitat is 
likely an over-simplification of their habitat requirements; however, since most recommendations for landscape management refer to the fragmentation or loss of forests in general (Ontario Partners in Flight 2005) and since I was also interested in a group of fragmentation-sensitive species, I feel this broad habitat definition is the most appropriate. Although, it is possible that a broad habitat definition may underestimate the absolute importance of fragmentation effects for individual species (Betts et al. 2006); this potential bias would be independent of the landscape size and therefore, will not affect my analysis of the change in relative effects with scale.

I used core forest to represent habitat fragmentation because it represents what are likely the two most important fragmentation-related mechanisms affecting forest birds in this region: 1) the presence and size of source populations in large areas of continuous forest (Nol et al. 2005) and 2) the inverse of the population's exposure to negative edge effects. These two mechanisms should combine for a strong positive effect of increasing percent core forest on fragmentation sensitive forest birds.

Road density was chosen to represent matrix quality because it both directly represents the potential increase in mortality due to collisions with vehicles and indirectly represents the increased mortality from collisions with human structures that are associated with roads (Erikson et al. 2005) as well as the increases in human activity, density of cats and other synanthropic predators, and human population density in areas with high road density. Agriculture is the dominant matrix type (i.e., non-forest landcover) in this region but spatially explicit measures of agricultural intensity are not available for my study area. Therefore, although it is possible that my analysis 
underestimates the absolute importance of matrix quality, my measure provides an unbiased estimate of how the relative importance of matrix quality varies across scales.

I used land cover data from the Ontario Land Cover Database (OMNR 1998), a Landsat TM-derived land cover classification with a 0.09 ha pixel resolution. The original 28 landcovers were reclassified into a binary habitat/non-habitat map where habitat was defined as forest cover, including 9 treed landcover types (including coniferous, deciduous, and mixed forest types). There has been little change in the distribution of forest cover and agriculture since the landcover data were collected in the mid-1990s (Dingle-Robertson 2007); however, some urban areas have expanded. Therefore, I updated the re-classified landcover using more recent information for urban development (OMNR 2007). The large size of my study area allowed me to sample larger landscapes, a greater range in variation for each landscape variable, and a more similar

range of variation across all spatial scales than is usually possible (e.g., compare Dunford and Freemark 2005; Renfrew and Ribic 2008).

\section{Response Variables}

I used Poisson regression to model the species richness of fragmentation-sensitive forest birds and logistic regression to model the occurrence (observed vs. unobserved) of the two most common fragmentation-sensitive species (Ovenbird [Seiurus aurocapilla] and Veery [Catharus fuscescens]) as functions of habitat amount, habitat fragmentation, and matrix quality. These fragmentation-sensitive species included 13 species (Ovenbird, Veery and 11 others, Table 3.1), whose ranges overlap the entire study area and that have, in previous studies, shown significant negative responses to forest fragmentation after controlling for forest amount. I used the richness of this functional group fragmentation-sensitive species - to represent the cumulative effects of increasing 
fragmentation (i.e., a decrease in Core) on forest bird diversity, because these species have shown similar responses to increasing forest fragmentation. I chose this cumulative response over individual species' responses for the remaining 11 species for two reasons. First, a Poisson regression model has greater statistical power than each of the singlespecies logistic models for the less common species, which maximizes my ability to measure changes in effects across scales. Second, because any management or modification of forest fragmentation in a landscape will presumably affect all of these sensitive species, their cumulative response is clearly important in a management/conservation context.

Forest bird data were taken from the point count database of the second Ontario Breeding Bird Atlas (OBBA). Point counts were conducted during peak breeding seasons from 2001 through 2005, by skilled volunteer observers using a standardized methodology (OBBA 2001). All birds seen or heard were recorded within a radius of 100 $\mathrm{m}$, during a single 5 minute period. Counts were conducted between dawn and 5 hours after sunrise and only in suitable weather. Count locations for the atlas project either followed a stratified-random distribution along roads within cells of a $10 \times 10 \mathrm{~km}$ grid (roadside counts) or were chosen by observers to ensure a representative sampling of landcover types with limited road access (off-road counts). Each location was sampled once over the 5 year atlas project.

To account for the effects of local habitat on bird distribution and detectability (i.e., probability of detecting a species given that it is present), I standardized the area of habitat within the point count plot and statistically controlled for differences between roadside and off-road counts. To standardize the area of forest sampled within each point 
count, I selected 2951 point counts (from among the $>30,000$ in my study area from the atlas database), for which $>95 \%$ of the $100 \mathrm{~m}$ radius count area was forest (i.e., point counts were centered approximately $100 \mathrm{~m}$ or more from a forest edge). This selection also controlled for potential variation among sites in species detectability because although detection probabilities are never $=1.0$, they are generally similar among different forest types (Lichstein et al. 2002) and they are generally very high for most of the species tested here (e.g., Farnsworth 2002). In addition, I tested for and found no relationship between the forest types inside the point counts and my landscape predictors, so that even if detectability had varied with forest type, it would not confound my analysis. Finally, I statistically controlled for differences between roadside $(\mathrm{N}=1221)$ and off-road counts $(\mathrm{N}=1730)$, which should account for most other detectability or local habitat effects on the bird observations.

Although I have done much to limit any potential bias differential detectability among species may still confound my analysis because I cannot directly measure it with my dataset. However, no dataset currently exists that would allow me to test my hypothesis and concurrently account for varying detection probabilities. I modelled the unadjusted count of fragmentation-sensitive species observed at each count location. All methods for estimating detectability require multiple sampling events (either in space or time, Boulinier et al. 1998, Mackenzie et al. 2006). Combining observations from multiple locations in my dataset would make it impossible to test my hypothesis of scale dependence. Data of multiple counts over time at the same locations (such as the North American Breeding Bird Survey) simply do not exist with comparable spatial resolution ( $\sim 3000$ forested count locations) and extent (spread across $210000 \mathrm{~km} 2)$. My estimate of 
the number of fragmentation sensitive species is better thought of as an index of the species present at individual sampling locations and not an estimate of the pure species richness of the bird community.

\section{Statistical Analyses}

Estimating the relative effects of correlated predictor variables in a regression model requires a trade-off between statistical power and accurate estimates of effect strength. Including correlated predictors increases type II errors by increasing the standard error of partial coefficients (Neter et al. 1990). However, removing predictors simply because they are highly correlated with others can lead to biased coefficient estimates and poor model fit (Neter et al. 1990, Smith et al. 2009). In addition, statistical approaches to dealing with correlations among landscape predictors, such as residual regression and variance partitioning approaches give biased estimates of relative importance (Smith et al. 2009). I have included correlated predictors because they represent distinct ecological mechanisms that likely influence forest birds in this region and removing one would lead to biased estimates of relative importance for the remaining predictors. I believe it is more appropriate to use a less powerful but unbiased design than to increase statistical power at the cost of biased and inaccurate estimates of effect strength.

I used three complementary analyses to ensure my results were not overly dependent on statistical artefacts (Koper et al. 2007 and Smith et al. 2009). I compared results from 1) the global model, 2) the most parsimonious model, and 3) the average of all supported models in a multi-model inference framework (Burnham and Anderson 2002, hereafter MMI). For the global model, I compared the partial regression coefficients and statistical significance within a model including all three predictors, an interaction term between amount and fragmentation (Forest*Core), and the covariates 
listed in the following paragraph. For the most parsimonious model, I compared the partial regression coefficients of the model with the lowest AIC from among all possible combinations of the 3 predictors and the interaction (the covariates were retained in all models). For the MMI analysis, I compared the summed Akaike weights of each predictor and the averaged partial regression coefficients from all models within a $95 \%$ confidence set. Similar results from a global model, the most parsimonious model, and averaged results for all supported models give me extra confidence that my estimates of relative importance are meaningful (MacNally 2000). For example, if the coefficient of one variable changes a great deal with the inclusion or exclusion of another, differences among the results of the three approaches will reflect that variation. I chose these three approaches because they are relatively familiar statistical tools and with the exception of summed Akaike weights, they give unbiased estimates of relative effect strength even with highly correlated predictors (Smith et al. 2009). The partial coefficients from all three analysis methods can be directly compared because the original predictors were standardized to a mean of 0 and a standard deviation of 1 .

I have presented plots only for the averaged coefficients from the MMI analyses because results from the three statistical analyses were so similar that the remaining plots of coefficients from the global and the most parsimonious models were almost indistinguishable. In addition, I have not reported confidence intervals associated with the coefficients in the most parsimonious models because they are very similar to those from the averaged MMI analyses that are shown in the plotted results.

\section{Controlling for spatial autocorrelation and other covariates}

I controlled for the effects of spatial autocorrelation, roadside survey biases, time of day, date, year, and habitat heterogeneity but including predictors representing these effects in 
all analyses (including all candidate models in the MMI and most parsimonious analyses). I controlled for the effects of spatial autocorrelation by using spatial eigenvector mapping to remove all significant autocorrelation from the residuals of global models (Griffith and Peres-Neto 2006). I also controlled for differences between off-road and roadside counts and for effects of time of day, date (both a linear and quadratic term), and differences among years. Finally, I controlled for habitat heterogeneity (number of forest classes) because it may influence my response variables through mechanism such as landscape complementation for individual species (Dunning et al. 1992) or habitat diversity for species richness, which act independently of habitat amount, fragmentation, or matrix quality. All of these covariates were somewhat correlated with at least one of the main predictors at some of the spatial scales. They were therefore included in all models at all scales to allow me to compare the primary predictors under similar conditions across scales.

\section{$3.4 \quad$ Results}

\section{Relative Importance across Scales}

The effects of habitat amount were positive and excluded zero from the confidence intervals for all three response variables at almost all scales (Table 3.2).

Forest amount became more important as a larger landscape was considered and was the most important predictor for all response variables in landscapes $>500 \mathrm{~m}$ in radius (Figures 3.2, 3.3, and 3.4). Habitat amount was present in the most parsimonious model at all scales and for all three response variables (Table 3.2). In fact, habitat amount was present in all supported models (summed Akaike weight $=1$ ) for all responses and at all scales except for Veery in $500 \mathrm{~m}$ landscapes (Table 3.3). 
The effects of fragmentation (Core) were more variable across scales and among responses. The relative ranking of fragmentation with other predictors changed with scale; for the number of fragmentation sensitive species and for Veery, core forest area was the most important predictor at very fine scales (500 m radius, larger coefficients in the global and most parsimonious models, Table 3.2, and larger averaged coefficients across all supported models, Figures 3.2 and 3.4, as well as higher Akaike weights, Table 3.3) but was less important at broader scales ( $2-10 \mathrm{~km}$ radius). For Ovenbird occurrence, Core was not important at any scale (Tables 2 and 3). For Veery, even the qualitative effect of fragmentation was scale dependent; core forest had a strong positive effect at fine scales and a strong negative effect at broad scales (Figure 3.4).

The effects of matrix quality were relatively weak and not strongly scale dependent. The standardized coefficient for road density was generally smaller than for either habitat amount or fragmentation (Figure 3.2), it was absent from the most parsimonious model for Ovenbird at all scales, for Veery at two of the five scales (Table 3.2), and was almost always missing from one or more of the supported models (summed Akaike weight $<1$, Table 3.3 ). Road density was somewhat important for the number of fragmentation sensitive species and for this response, its effects were generally consistent across scales. It had a consistently negative effect and it was included in the most parsimonious model at all scales (Figure 3.2 and Table 3.3).

In all cases, the interaction between habitat amount and fragmentation (Forest*Core) had the expected qualitative effect; increasing core forest had a stronger positive effect when amount was low (Figure 3.2). The interaction was only scale dependent for Veery. It was important in landscapes $1-5 \mathrm{~km}$ in radius (Figure 3.4), 
where it was included in the best model (Table 3.2) and its summed Akaike weight was highest (0.91 - 1 Table 3.3$)$ but not important in very small or very large landscapes (Figure 3.4). For the number of species it was important at all scales (Figure 3.2, included in the best model and most of the supported models at all scales Tables 2 and 3). For Ovenbird it was not important at any scale (Figure 3.3 and Tables 2 and 3).

\section{Predictor variance and covariance}

The overall correlation among the predictors increased slightly at broader landscape scales (Table 3.4). Average VIF was $<3$ at all scales and VIF for single predictors was always $<4$ (VIF $>10$ indicates severe collinearity, Neter et al. 1990). The increase in collinearity in larger landscapes is also reflected in the larger error bars around coefficient estimates at broad scales (Figure 3.2). Most of the increasing collinearity with scale (and therefore the increasing error around coefficient estimates) seems to be due to an increase in the strength of correlation between Core and Road (from -0.19 to -0.62 ). I am confident that this increasing correlation has not strongly influenced my results because the level of correlation is not particularly high even in large landscapes and the confidence limits around the coefficients for Core and Road do not increase with scale at similar rates (Figures $3.2-3.4$ ).

\subsection{Discussion}

Habitat amount in the surrounding landscape had an important and consistently positive effect on forest birds regardless of the size of the landscape considered but the strength of the effect increased with the size of the landscape. For most of the scales tested here, my results agree with previous studies, which have shown that habitat amount is more important than fragmentation in determining species distributions (Betts et al. 2006; Smith et al. 2009; and others reviewed in Fahrig 2003). The novel 
contribution of this study is that the positive effect of habitat amount (at least for fragmentation sensitive forest birds) is consistent over more than one order of magnitude in landscape radius $(0.5 \mathrm{~km}-10 \mathrm{~km})$ or more than two orders of magnitude in landscape area $\left(\sim 1 \mathrm{~km}^{2}-300 \mathrm{~km}^{2}\right)$ but this is not the case for the effects of fragmentation. Even considering the interaction between habitat amount and fragmentation, which complicates a simple interpretation of relative importance, the effects of habitat amount were more important and more consistent across a wide range of values of fragmentation; while the effects of fragmentation tended to be weaker or even change direction depending on the value of habitat amount (e.g., for species richness, the effects of core forest were positive at low levels of forest amount and neutral or negative at high levels, Figure 3.2). With a correlative study such as this it is difficult to tell whether the process(es) through which habitat amount influences biodiversity are constant across these scales or that the processes change but their resultant effects are constant; however, the general implications are clear - a reduction in habitat amount will likely have a negative effect on fragmentation sensitive forest bird species at all these scales.

As predicted, fragmentation effects depended more on landscape scale. My results lend support to the hypothesis that the multiple processes through which fragmentation affects populations and communities act at different scales and therefore; the relevant positive, negative, or neutral effects of fragmentation are evident at the different scales (Addicott et al. 1987). The size of the landscape considered affected the relative importance, absolute importance (significance, inclusion in the best model), and even the qualitative effect of increasing fragmentation. Interestingly, the effects of fragmentation changed with scale for a group of forest bird species that are generally considered 
sensitive to the effects of fragmentation. If fragmentation sensitive species can appear tolerant of fragmentation at some spatial scales, it suggests that species previously thought to be tolerant may need to be reassessed. Further, if the direction of a species' response to habitat fragmentation depends on the scale of the landscape considered, the very notion of classifying species by their sensitivity to fragmentation may be unproductive.

If fragmentation effects are generally more scale dependent than those of habitat amount, it may explain some of the conflicting results around the relative effects of habitat loss and fragmentation in the literature (Fahrig 2003; Koper et al. 2007; and Smith et al. 2009). Previous studies on forest birds have used landscapes ranging in size from 2.5-3 $\mathrm{km}^{2}$ (McGarigal and McComb 1995) through $100 \mathrm{~km}^{2}$ (closest in area to my $5 \mathrm{~km}$ radius landscapes, Trzinski et al. 1999; Radford and Bennett 2007, Table 3.1). Additionally, this scale dependence may partly explain why particular species have shown conflicting responses to fragmentation in different studies. For example, the Scarlet Tanager showed a significant negative response to increasing fragmentation within $6 \mathrm{~km}^{2}$ and $12 \mathrm{~km}^{2}$ landscapes in Villard et al. 1999 and Austen et al. 2001, but a significant positive response within the $100 \mathrm{~km}^{2}$ landscapes in Trzcinski et al. 1999.

However, scale dependence cannot definitively explain all differences among studies. Fragmentation effects may also depend on other study-specific factors such as the metric used to quantify fragmentation (McGarigal and Cushman 2002) or the statistical method used to estimate the effects (Smith et al. 2009). For example, I did not find a significant response to increasing fragmentation for Ovenbirds at any scale; which contrasts with a significantly negative response to increasing fragmentation in the $12 \mathrm{~km}^{2}$ 
landscapes of Betts et al. 2006 and a significantly positive response in the relatively similarly sized $6 \mathrm{~km}^{2}$ landscapes of Villard et al. 1999. Different fragmentation metrics may be better or worse representations of the important ecological processes that are sensitive to fragmentation. In my study I used core forest area to represent relatively broad scale processes such as the presence of source populations and negative edge effects. However, if conspecific attraction is an important process (Fletcher 2009), then a patch-related variable such as patch size may have given different results. Ultimately, my finding that fragmentation effects depended more on spatial scale than those of habitat amount supported my hypothesis: The potentially conflicting processes influenced by fragmentation, which act at different spatial scales, create a greater scale dependency for fragmentation effects than for habitat amount effects. However, I have only sampled a small number of forest bird species and it remains to be seen if there is broad support for this hypothesis in other taxa.

The effects of matrix quality, as measured here (road density), were generally weak; therefore, it is difficult to derive any strong inferences on their scale dependence. My results differ from those of a previous study that showed an increasing importance of matrix quality with landscape size (Dunford and Freemark 2005). This may be because I did not include sufficiently broad spatial scales (i.e., $>10 \mathrm{~km}$ radius) for my particular measure of matrix quality and/or my measure of matrix quality may not represent the same agricultural processes measured in Dunford and Freemark (2005). I chose road density as a variable that should represent the increased probability of mortality within poor-quality matrix - both directly through increased collisions with vehicles and as a proxy for factors that are associated with roads such as the density of buildings and 
towers that increase bird mortalities (Erikson et al. 2005). However, because many of these mortalities occur during migration, when many of these forest birds are moving at very large spatial scales, their effects may only be apparent at even broader spatial scales than those measured here. Additionally, many different measures of matrix quality are possible and some may not represent the matrix-dependent processes that are important for forest birds. For example, road density likely does not represent the same processes measured by an estimate of agricultural intensity in Dunford and Freemark (2005). Agricultural activities influence the density of nest parasites (Hoover and Brittingham 1993), predators (Burke et al. 2004), or the rates of chemical inputs from agricultural activities (Stinson et al. 1994). Unfortunately, data on agricultural activities that might better represent these processes are not available in the study area at spatial resolutions comparable to the road or landcover data used here.

My findings also support the existence of a fragmentation threshold, where the negative effects of fragmentation become more important when the amount of habitat is low (Andren 1994). This threshold has been supported by theoretical work (e.g., Fahrig 1998) but has not been frequently demonstrated in empirical systems (Fahrig 2003 but see Betts et al. 2007). In my study, whenever fragmentation effects were somewhat important and in the expected direction (positive effect of increasing core forest), there was evidence of a statistical interaction between amount and fragmentation (although very limited for Veery at $500 \mathrm{~m}$ ). The direction of the interaction indicated that the effects of core forest were more positive (i.e., the effects of fragmentation were more negative) when forest amount was low. 
In this study, I have shown that the effects of fragmentation on forest birds appear to be more scale dependent than the effects of habitat amount. However converting the quantitative results (standardized coefficients) of this focal-plot study into specific landscape recommendations is not simple. In my analyses, I used the same response variable measured at a central plot for all landscape scales. This was necessary so that the response variable remained constant across the different spatial scales and I could therefore be sure that a change in effect across scale truly was a function of changes in the landscape measures. However, these effects on a response measured in a focal plot may not directly translate to effects on the bird community measured within an entire landscape, which is ultimately the goal of landscape management. For example, my response variables were measured only within forest areas that were $>100 \mathrm{~m}$ in radius (to control for local habitat and sampling effects); however, the landscape of southern Ontario includes a great deal of forest that does not fulfil this criterion. It remains to be seen whether bird populations and communities measured over the entire landscape will respond similarly to measures of habitat amount, fragmentation, and matrix quality in landscapes of varying size.

Although translating the quantitative results here into specific recommendations for management is complicated, these results do suggest three broad conclusions relevant to managing scale-dependent landscape effects. First, if fragmentation effects are only apparent at some scales then these effects should only be managed or considered at that scale. Second, if the relative ranking of fragmentation and amount changes with scale (e.g., as it did for the number of fragmentation-sensitive species in this study), then the ranking of conservation options will depend on the scale of influence of the managing 
organization. Third, if fragmentation has positive effects at one scale and negative effects at another (e.g., Veery in this study), then in this context, managing fragmentation may not be an effective use of conservation resources. 


\section{6}

Tables

Table 3.1. Species with previously demonstrated, significantly negative responses to increasing forest fragmentation, independent of the effects of habitat loss. *Four species showed significantly negative responses in more than one study.

\begin{tabular}{|c|c|c|c|}
\hline Species & Response to Fragmentation & Reference & $\begin{array}{l}\text { Landscape } \\
\text { Size used in } \\
\text { Study } \\
\end{array}$ \\
\hline $\begin{array}{l}\text { Black-throated Green Warbler } \\
\text { (Dendroica virens) }\end{array}$ & $\begin{array}{l}\text { Positive effect of core area and patch } \\
\text { size after controlling for habitat } \\
\text { amount }\end{array}$ & Austen et al. 2001 & $12 \mathrm{~km}^{2}$ \\
\hline $\begin{array}{l}\text { Hairy Woodpecker } \\
\text { (Picoides villosus) }\end{array}$ & Negative effect of edge length & Villard et al. 1999 & $6 \mathrm{~km}^{2}$ \\
\hline $\begin{array}{l}\text { Hermit Thrush } \\
\text { (Catharus guttatus) }\end{array}$ & $\begin{array}{l}\text { Negative effect of principle } \\
\text { component representing increasing } \\
\text { fragmentation }\end{array}$ & $\begin{array}{c}\text { Trzcinski et al. } \\
1999\end{array}$ & $100 \mathrm{~km}^{2}$ \\
\hline $\begin{array}{l}\text { Least Flycatcher } \\
\text { (Empidonax minimus) }\end{array}$ & Negative effect of edge length & Villard et al. 1999 & $6 \mathrm{~km}^{2}$ \\
\hline $\begin{array}{l}\text { Northern Waterthrush } \\
\text { (Seiurus noveboracensis) }\end{array}$ & $\begin{array}{l}\text { Positive effect of core area and patch } \\
\text { size after controlling for habitat } \\
\text { amount }\end{array}$ & Austen et al. 2001 & $12 \mathrm{~km}^{2}$ \\
\hline \multirow{2}{*}{$\begin{array}{l}\text { Ovenbird } \\
\text { (Seiurus aurocapilla) } \\
\text { Pine Warbler } \\
\text { (Dendroica pinus) }\end{array}$} & $\begin{array}{l}\text { Positive effect of patch size after } \\
\text { controlling for habitat amount }\end{array}$ & Betts et al. 2006 & $12 \mathrm{~km}^{2}$ \\
\hline & $\begin{array}{l}\text { Positive effect of patch size after } \\
\text { controlling for habitat amount }\end{array}$ & Austen et al. 2001 & $12 \mathrm{~km}^{2}$ \\
\hline \multirow{2}{*}{$\begin{array}{l}\text { Pileated Woodpecker* } \\
\text { (Dryocopus pileatus) }\end{array}$} & Positive effect of core area & Austen et al. 2001 & $12 \mathrm{~km}^{2}$ \\
\hline & $\begin{array}{l}\text { Negative effect of mean Nearest } \\
\text { Neighbour Distance }\end{array}$ & Villard et al. 1999 & $6 \mathrm{~km}^{2}$ \\
\hline $\begin{array}{l}\text { Purple Finch } \\
\text { (Carpodacus purpureus) }\end{array}$ & $\begin{array}{l}\text { Negative effect of principle } \\
\text { component representing increasing } \\
\text { fragmentation }\end{array}$ & $\begin{array}{c}\text { Trzcinski et al. } \\
1999\end{array}$ & $100 \mathrm{~km}^{2}$ \\
\hline \multirow{2}{*}{$\begin{array}{l}\text { Scarlet Tanager* } \\
\text { (Piranga olivacea) }\end{array}$} & $\begin{array}{l}\text { Positive effect of patch size after } \\
\text { controlling for habitat amount }\end{array}$ & Austen et al. 2001 & $12 \mathrm{~km}^{2}$ \\
\hline & & Villard et al. 1999 & $6 \mathrm{~km}^{2}$ \\
\hline \multirow{2}{*}{$\begin{array}{l}\text { Veery* } \\
\text { (Catharus fuscescens) }\end{array}$} & $\begin{array}{l}\text { Positive effect of patch size after } \\
\text { controlling for habitat amount }\end{array}$ & Austen et al. 2001 & $12 \mathrm{~km}^{2}$ \\
\hline & Negative effect of edge length & Villard et al. 1999 & $6 \mathrm{~km}^{2}$ \\
\hline \multirow[b]{2}{*}{$\begin{array}{l}\text { Yellow-bellied Sapsucker* } \\
\text { (Sphyrapicus varius) }\end{array}$} & Positive effect of core area & Austen et al. 2001 & $12 \mathrm{~km}^{2}$ \\
\hline & $\begin{array}{l}\text { Negative effect of principle } \\
\text { component representing increasing } \\
\text { fragmentation }\end{array}$ & $\begin{array}{c}\text { Trzcinski et al. } \\
1999\end{array}$ & $100 \mathrm{~km}^{2}$ \\
\hline $\begin{array}{l}\text { Yellow-rumped Warbler } \\
\text { (Dendroica coronata) }\end{array}$ & $\begin{array}{c}\text { Negative effect of principle } \\
\text { component representing increasing } \\
\text { fragmentation }\end{array}$ & $\begin{array}{l}\text { Trzcinski et al. } \\
1999\end{array}$ & $100 \mathrm{~km}^{2}$ \\
\hline
\end{tabular}


Table 3.2. Standardized coefficients from the most parsimonious model (lowest AIC) within landscapes of 5 different sizes. Predictors included 3 landscape variables - total amount of forest (Forest), the proportion of forest area within the core of large forests (Core), and the density of roads (Road) - plus the Forest*Core interaction. Models predicted the number of fragmentation sensitive species observed (Poisson regression) and the occurrence (logistic regression) of Ovenbird and Veery at 2951 forested point counts in Southern Ontario Canada. Candidate models included all 8 possible combinations of the 3 landscape predictors, plus the Forest ${ }^{*}$ Core interaction. All models included covariates controlling for spatial autocorrelation, time of day, season, habitat heterogeneity, and roadside survey biases.

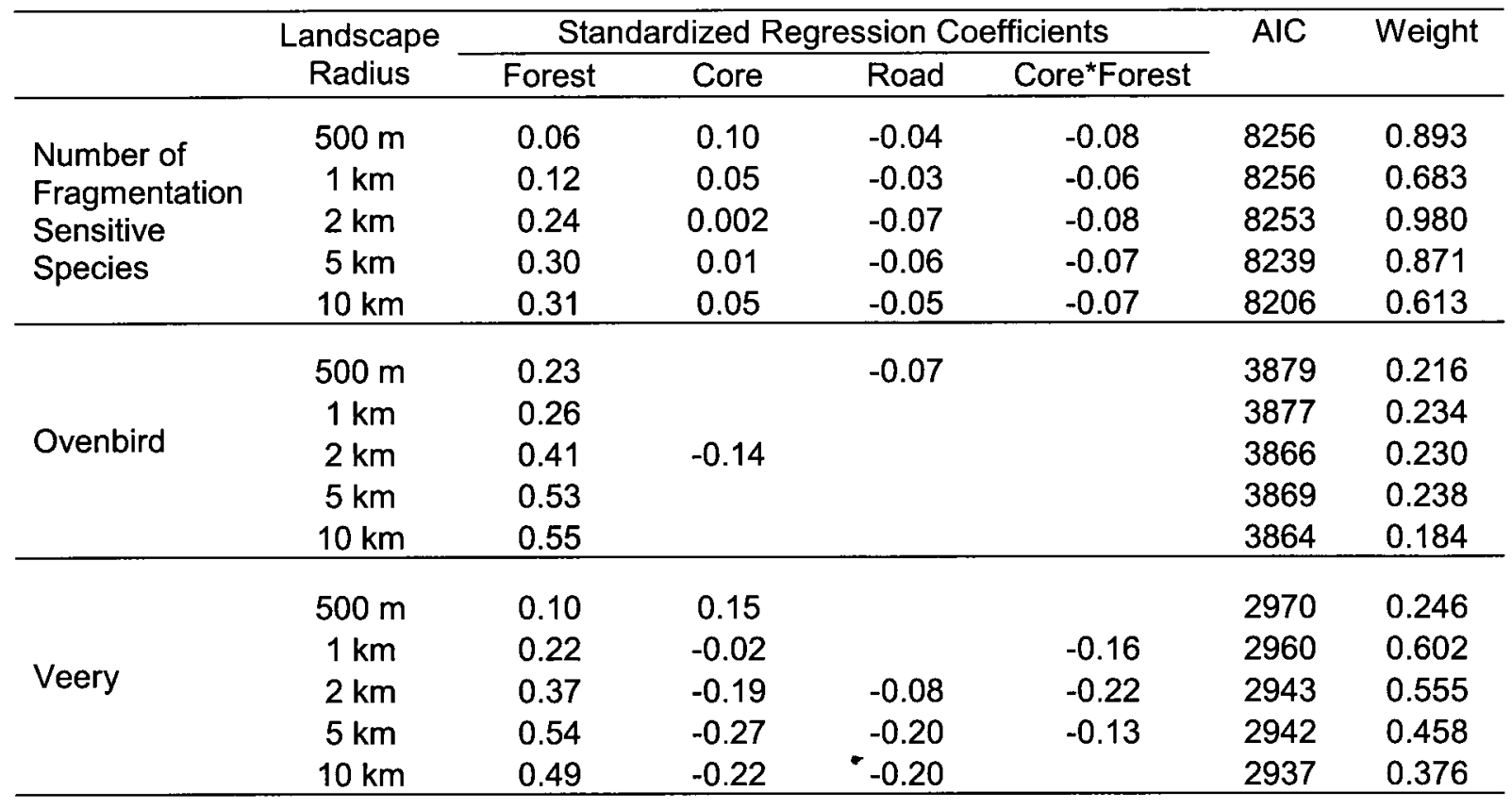


Table 3.3. Summed Akaike weights for the 3 landscape predictors - total amount of forest (Forest), the proportion of forest area within the core of large forests (Core), and the density of roads (Road), plus the Forest*Core interaction - measured within landscapes of 5 different sizes. Models predicted the number of fragmentation sensitive species observed (Poisson regression) and the occurrence (logistic regression) of Ovenbird and Veery at 2951 forested point counts in Southern Ontario, Canada. Weights were summed across the $95 \%$ confidence set of supported models from among the same competing models as in Table 3.2.

\begin{tabular}{lccccc}
\hline & Landscape & \multicolumn{5}{c}{ Summed Akaike Weights } \\
\cline { 3 - 6 } & Radius & Forest & Core & Road & Core*Forest \\
\hline \multirow{2}{*}{ Number of } & $500 \mathrm{~m}$ & 1 & 1 & 0.89 & 1 \\
Fragmentation & $1 \mathrm{~km}$ & 1 & 1 & 0.70 & 0.97 \\
Sensitive & $2 \mathrm{~km}$ & 1 & 1 & 1 & 1 \\
Species & $5 \mathrm{~km}$ & 1 & 1 & 0.87 & 1 \\
& $10 \mathrm{~km}$ & 1 & 1 & 0.62 & 1 \\
\hline & $500 \mathrm{~m}$ & 1 & 0.51 & 0.75 & 0.34 \\
& $1 \mathrm{~km}$ & 1 & 0.40 & 0.36 & 0.08 \\
Ovenbird & $2 \mathrm{~km}$ & 1 & 0.88 & 0.36 & 0.38 \\
& $5 \mathrm{~km}$ & 1 & 0.42 & 0.43 & 0.16 \\
& $10 \mathrm{~km}$ & 1 & 0.47 & 0.43 & 0.14 \\
\hline & & & & & \\
& $500 \mathrm{~m}$ & 0.71 & 0.96 & 0.26 & 0.32 \\
Veery & $1 \mathrm{~km}$ & 1 & 1 & 0.29 & 1 \\
& $2 \mathrm{~km}$ & 1 & 1 & 0.56 & 1 \\
& $5 \mathrm{~km}$ & 1 & 1 & 0.49 & 0.91 \\
& $10 \mathrm{~km}$ & 1 & 0.98 & 0.38 & 0.40 \\
\hline
\end{tabular}


Table 3.4. Collinearity among the landscape predictors increased with landscape size. Variance inflation factors (VIF) and pair-wise Pearson correlation coefficients for predictors: total amount of forest (Forest), the proportion of forest area within the core of large forests (Core), and the density of roads (Road). VIF represents the predictor's overall collinearity in a regression model with all other landscape predictors, the interaction between Forest and Core, and all covariates (see Table 3.2 for a list of covariates). Values above the diagonal are for large landscapes (10 $\mathrm{km}$ radius) and below are for small landscapes $(0.5 \mathrm{~km}$ radius). Values for intermediate scales are intermediate to those shown here.

\begin{tabular}{|c|c|c|c|c|}
\hline & \multicolumn{3}{|c|}{ Largest Landscapes (radius $=10 \mathrm{~km}$ ) } \\
\hline & & Forest (VIF $=2.5$ ) & Core (VIF = 3.2) & Road (VIF $=1.2)$ \\
\hline \multirow{3}{*}{$\begin{array}{l}\text { Smallest } \\
\text { Landscapes } \\
\text { (radius = } \\
500 \mathrm{~m} \text { ) }\end{array}$} & Forest $(\mathrm{VIF}=1.9)$ & & 0.64 & -0.25 \\
\hline & Core (VIF = 1.9) & 0.68 & & -0.62 \\
\hline & Road (VIF = 1.1) & -0.16 & -0.19 & \\
\hline
\end{tabular}




\section{7}

Figures

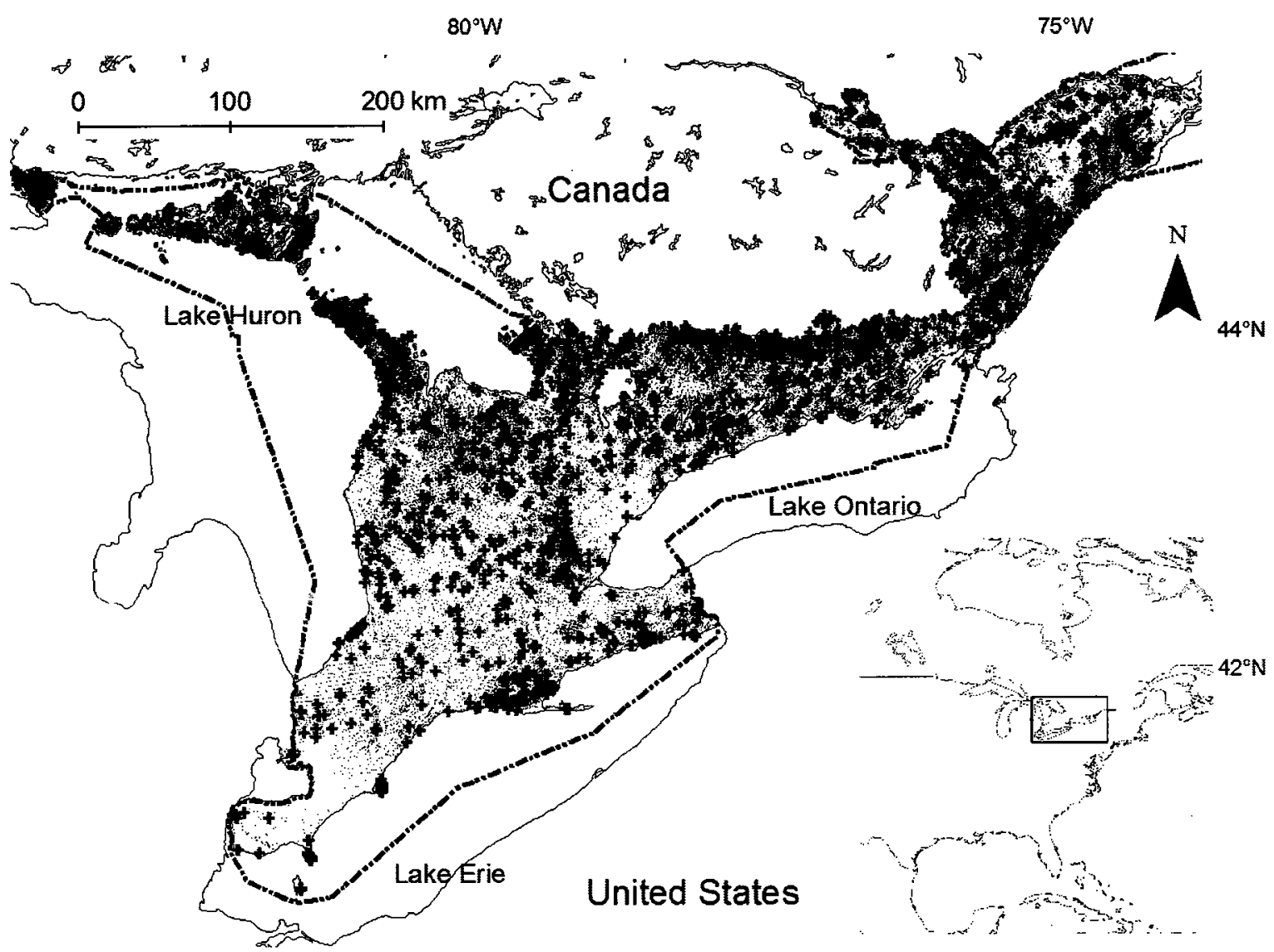

Figure 3.1. Distribution of forest cover (grey areas) and 2951 point count sampling stations with $>95 \%$ forest cover inside the $100 \mathrm{~m}$ radius sampling area (crosses), within the study area (portion of Bird Conservation Region 13 in Ontario, Canada). 


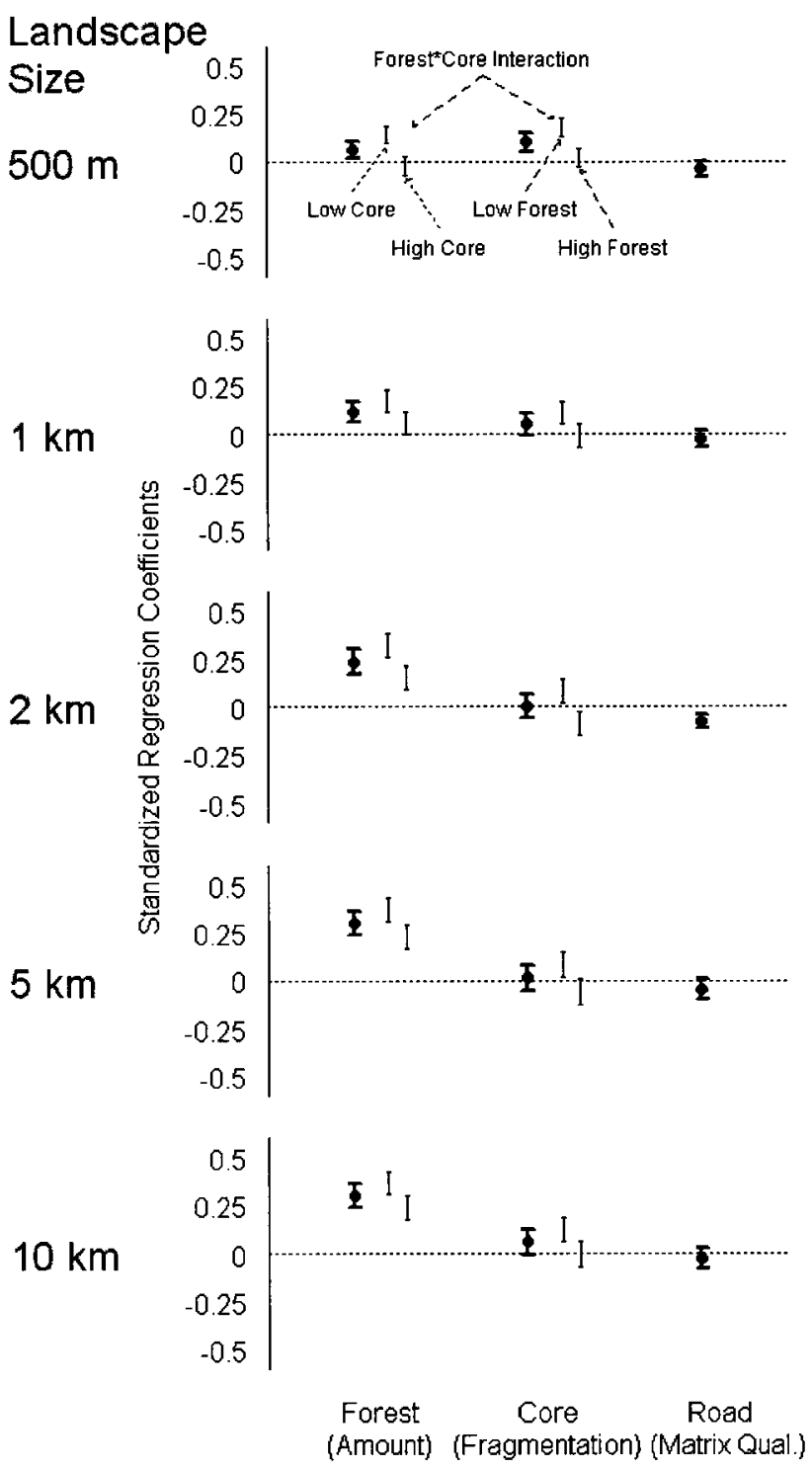

Figure 3.2. Poisson regressions coefficients for the effects of the total amount of forest (Forest), the proportion of forest area in large core forests (Core), and the density of roads (Road) in landscapes of 5 different radii on the number of fragmentation sensitive bird species observed at forested point counts in Southern Ontario Canada. Points are the standardized, averaged coefficients (weighted by the model's Akaike weight) from all models in a $95 \%$ confidence set and error bars are the associated unconditional confidence limits. An interaction between Forest and Core, is demonstrated by the error bars without points. These error bars represent the confidence limits around the coefficient for Forest at low and high levels of Core ( -1 and +1 standard deviation of Core) and for Core at low and high levels of Forest ( -1 and +1 standard deviation of Forest). All models accounted for the effects of spatial autocorrelation, time of day, season, surrounding habitat diversity, and roadside survey biases 


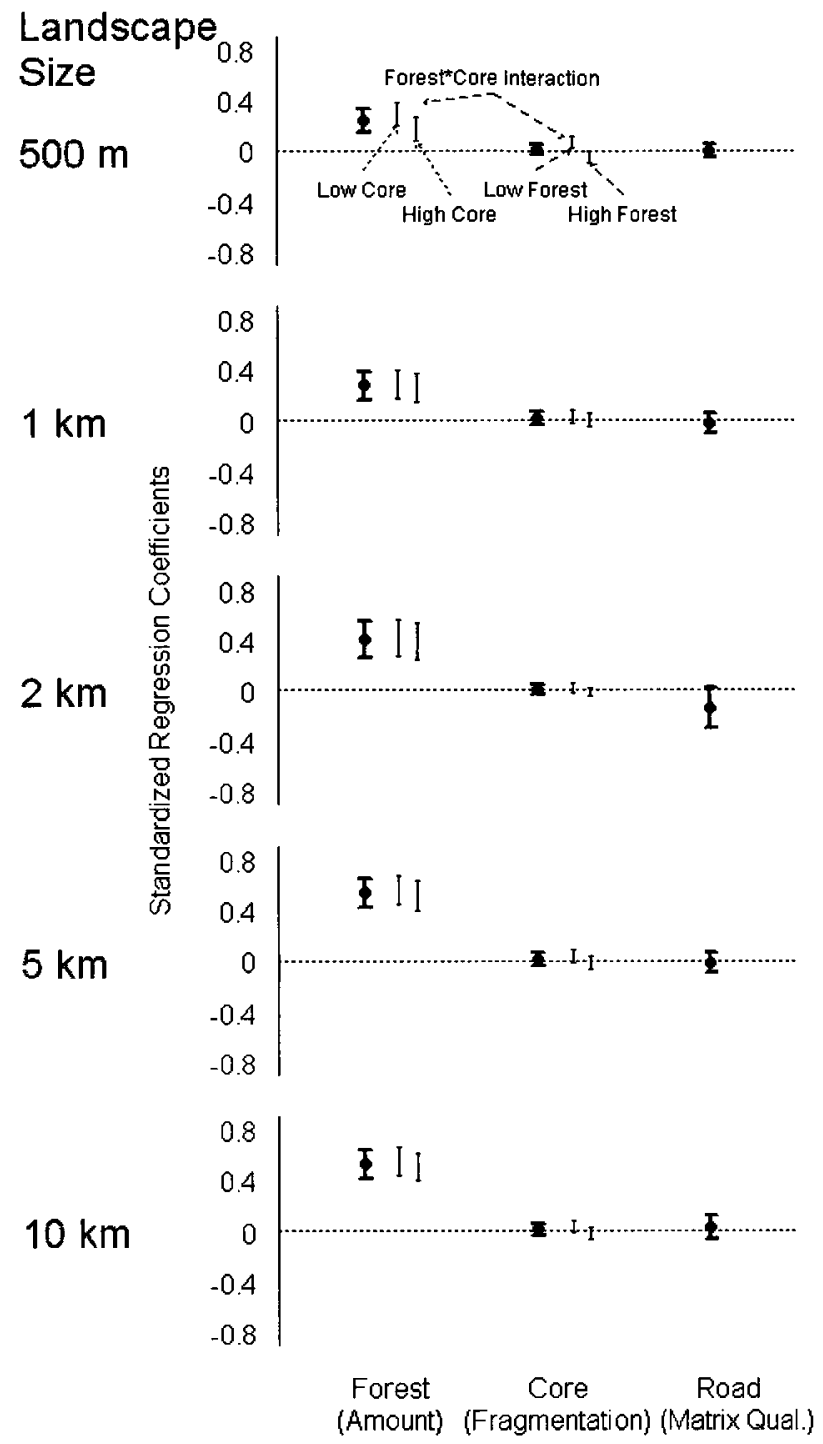

Figure 3.3. Logistic regressions coefficients for the effects of the total amount of forest (Forest), the proportion of forest area in large core forests (Core), and the density of roads (Road) in landscapes of 5 different radii on the occurrence of Ovenbird at forested point counts in Southern Ontario Canada. Points are the standardized, averaged coefficients (weighted by the model's Akaike weight) from all models in a $95 \%$ confidence set and error bars are the associated unconditional confidence limits. An interaction between Forest and Core, is demonstrated by the error bars without points. These error bars represent the confidence limits around the coefficient for Forest at low and high levels of Core $(-1$ and +1 standard deviation of Core) and for Core at low and high levels of Forest ( -1 and +1 standard deviation of Forest). All models accounted for the effects of spatial autocorrelation, time of day, season, surrounding habitat diversity, and roadside survey biases 


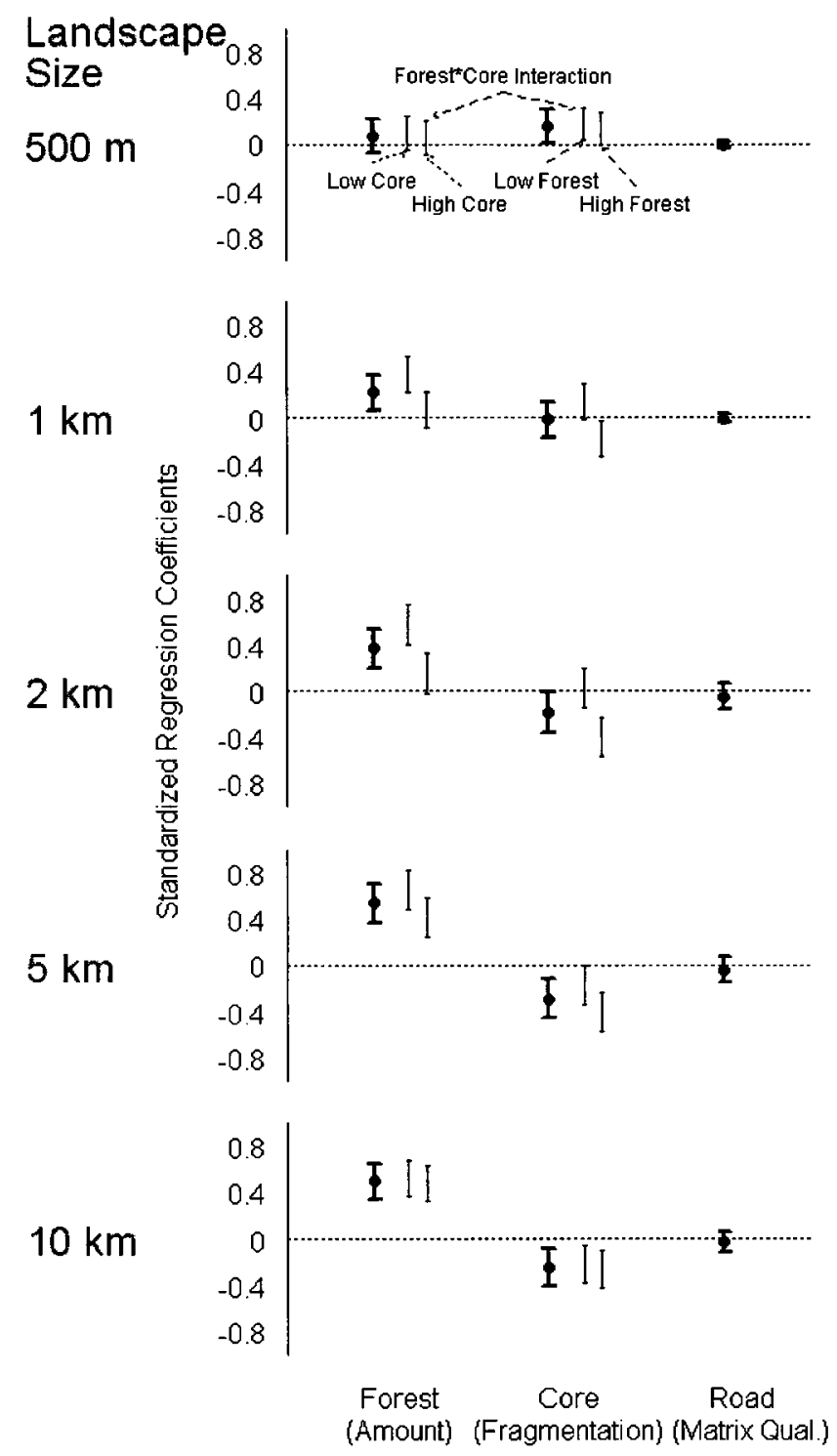

Figure 3.4. Logistic regressions coefficients for the effects of the total amount of forest (Forest), the proportion of forest area in large core forests (Core), and the density of roads (Road) in landscapes of 5 different radii on the occurrence of Veery at forested point counts in Southern Ontario Canada. Points are the standardized, averaged coefficients (weighted by the model's Akaike weight) from all models in a $95 \%$ confidence set and error bars are the associated unconditional confidence limits. An interaction between Forest and Core, is demonstrated by the error bars without points. These error bars represent the confidence limits around the coefficient for Forest at low and high levels of Core $(-1$ and +1 standard deviation of Core) and for Core at low and high levels of Forest (-1 and +1 standard deviation of Forest). All models accounted for the effects of spatial autocorrelation, time of day, season, surrounding habitat diversity, and roadside survey bias. 


\section{Chapter 4. How should we predict the relative impacts of management options in ecology and conservation?}

(Adapted from: How should we predict the relative impacts of management options in ecology and conservation? In Review)

\subsection{Chapter Summary:}

In practical applications of ecology and conservation, we must estimate the relative value of different management options, to make appropriate planning decisions with limited resources. This is often done by estimating the relative importance of predictors in a regression model. However, there are many different statistical tools available to measure relative importance in regression and, as a result, comparing or synthesising conclusions across studies is difficult. Indeed, in reviewing the literature I found eight different metrics were used to infer relative importance in a conservation context, measuring at least three fundamentally different kinds of statistical information (i.e., significance, explained variance, and effect strength). I used simulations to compare different metrics and evaluate them against five criteria that are necessary to compare conservation implications across studies: estimates should be unbiased with respect to 1) sample size, 2) range of variation sampled for each predictor, 3) collinearity among predictors, 4) presence of spurious predictors or 5) presence of suppressor effects. I found that the most useful measure of relative importance, from both a practical and theoretical standpoint, is a novel metric that I introduce here, proportional partial regression coefficients. These coefficients are partial regression coefficients from a thoughtfully constructed model that controls for potentially confounding factors, re-scaled to a proportion of the range of practical or theoretical limits to management. This metric meets all of the criteria and represents the relative potential benefit to conservation from managing an ecological factor. In contrast, measures of explained variation fail to meet most of the criteria and do 
not provide clear implications for conservation; while statistical significance and summed Akaike weights fail to meet any of the criteria and are not useful measures of relative importance. Using proportional partial regression coefficients will facilitate comparison across studies and ultimately, provide a more useful evidence-base for effective conservation.

\subsection{Introduction}

Measuring the relative importance of ecological factors and options for conservation/management is important for ecological understanding and for ranking competing management options (Wilson et al. 2007). A conservation manager, having to decide where to invest limited resources - e.g., in protecting more habitat, reducing mortality from predation, or controlling an invasive species - must have a way of estimating the potential benefit from each strategy (Sutherland et al. 2004). Although there may be evidence that each of the competing strategies has a statistically significant effect on the intended conservation goal, statistical significance does not mean managing that effect will be an efficient use of resources. For that, a cost-benefit analysis is required (e.g., Perhans et al. 2008), which involves estimating the expected benefits from the independent application of each strategy or management option (note: if the options, strategies, or ecological factors cannot be independently manipulated at all - i.e., there is a tight, causal or logical relationship between them - then questions of relative importance are trivial). In practice, this must often be done by measuring the relative importance of predictors in a regression model because manipulative experiments, on the relevant spatial and temporal scales for most conservation-related questions, are difficult or impossible (MacNally 2000). Even in situations where manipulative experiments are 
possible, a preliminary regression analysis can predict the likely outcomes and guide decisions about which experimental treatments to apply (James and McCulloch 1990). Currently, it is difficult to generalize the conservation implications of published studies comparing the relative importance of ecological factors or management options because those studies have used different ways of measuring relative importance. For example, I searched the Web of Science and found 97 papers that included: "relative importance" or a closely related term in the title; "conserve" or "ecolog" somewhere in the record text (including the abstract); and were included in the "ecology" or "biodiversity and conservation" subject areas. By reading the abstracts and/or the full text of these studies, I identified 59 that drew explicit conservation conclusions. These conclusions mostly focused either on single species management (e.g., predation vs hunting on a game bird, Watson et al. 2007; habitat quality vs fragmentation for an endangered plant, Adriaens et al. 2009) or landscape manipulations for communities (e.g., woodland cover vs configuration on birds, Radford and Bennett 2007; fine vs coarse woody debris on fungi, Norden et al. 2004), but all discussed the relative potential benefits of manipulating or managing one factor over another. I then reviewed the methods and results sections to determine which statistical methods were used to infer relative importance of predictor variables and found that it was measured using one or a combination of eight methods/metrics that estimate different properties of the regression model (Table 4.1).

I have grouped these eight metrics and others suggested in the statistical literature (e.g., Johnson and LeBreton 2004, Grace and Bollen 2005, Gromping 2007) into one of three categories (Table 4.1). First, coefficient-based metrics (hereafter "strength- 
metrics") estimate a regression coefficient on a common scale and represent a direct translation of change in the predictor into change in the response (Achen 1982). Predictors are considered more important if their strength-metric estimates are of larger absolute value. Second, variance partitioning-based metrics (hereafter "variancemetrics") estimate a fraction of the variation in the response that can be explained by the variation in each of the predictors. These fractions are often expressed as a proportion of the variance explained by a full model (i.e., a partitioning of the full model $\mathbf{R}^{2}$ ). Predictors with variance-metric estimates closer to 1 are considered more important than those with lower estimates. Finally, confidence-based metrics (hereafter "confidencemetrics") measure aspects of probability, error, or support in the data for a model (i.e., pvalues and summed Akaike weights). Some feel that confidence-metrics are improper measures of relative importance (Kruskal and Majors 1989). However, I have included them here because surveys of the literature show that they are frequently used to estimate relative importance (Kruskal and Majors 1989, Yoccoz 1991, Stephens et al. 2007, and $34 \%$ of the studies in my review, Table 4.1 ), and one - summed Akaike weights - is formally called a measure of "relative importance" (Burnham and Anderson 2002). Relative importance metrics have fundamental similarities, yet their differences are important enough to complicate inferences across studies. They are similar in that they are all based on a general or generalized linear model, so they share many mathematical characteristics and ultimately rest on the same set of statistical assumptions (e.g., that the linear model is a reasonable representation of the functional ecological relationship). However, they differ in their calculations and their underlying definitions of relative importance (Achen 1982). As a result, different metrics generate different 
quantitative estimates, and in some cases different qualitative rankings, when applied to the same data (Murray and Conner 2009, Smith et al. 2009). Therefore, published estimates of the relative importance of ecological factors or competing conservation options may reflect as much or more the statistical approach used in a particular study, as the true potential benefit to conservation from managing each factor.

To be useful for drawing generalizations and comparisons across studies, the best relative importance metrics should meet five criteria. They should provide unbiased estimates regardless of: variation in sample size, collinearity, and the range of variation in each predictor; as well as the inclusion of spurious predictors and the presence of suppressor effects. Obtaining a clear understanding would be difficult if replicated studies, measuring the relative importance of environmental factors $\mathrm{A}$ and $\mathrm{B}$ on a conservation response, were to have different results, only because they varied in properties of the particular sample (i.e., the sample size, the sampled ranges of A and B, or the level of correlation between A and B ). For example, Trzcinski et al. 1999, Villard et al. 1999, Austen et al. 2001, and Smith et al. in press, compared the effects of habitat amount and habitat fragmentation on forest birds in southern Ontario Canada. However, each of these four studies used one or a combination of three methods to draw their conclusions, including: standardized partial regression coefficients, simple regression coefficients, and statistical significance. Therefore, because these studies vary in: sample size, by almost two orders of magnitude ( $n=33$ vs $n=2500)$; correlation between the two key predictors $(r=0.42$ vs $r=0.91)$; and, the range of habitat amount sampled (15$45 \%$ vs $1-90 \%$ ), a direct comparison is difficult because at least some of these methods depend, to some degree, on these sample properties (Neter et al. 1990, Grace and Bollen 
2005). Additionally, metrics should be unaffected by the presence of spurious predictors - i.e., predictors that are statistically correlated with the response and other predictors but that have no causal influence. Finally, the most useful metrics should give unbiased estimates in the presence of suppressor effects (Cohen and Cohen 1983). Suppressor or masking effects occur when conflicting ecological factors cancel each other's effects. For example, increasing the amount of forest in a landscape may have a positive influence on population size through increasing habitat availability but the response may be masked if an associated increase in forest edge increases predation rates. These types of suppressor relationships are likely common in ecological systems, yet rarely explicitly considered in discussions of relative importance (although see Smith et al. 2009). The first three of these five criteria (that metrics are independent of: sample size, collinearity, and the sampled range of each predictor) test whether the metric depends on the properties of the statistical sample; the last two criteria (that metrics are unaffected by spurious predictors and suppressor effects) test whether the metric is biased by complexities that are relatively common in ecological analyses. The statistically-savvy reader will recognize in advance, that many of the metrics in table 4.1 will not meet all of these criteria. However, I believe that these are reasonable criteria for any estimate of the relative benefits of competing factors or management options in conservation, and that comparing and contrasting the metrics, under simulated conditions will help ecologists better understand each metric and choose among them.

In this study I used simulated data to compare and contrast the behaviours of a wide range of metrics of relative importance against five criteria. I included examples of all three categories of metrics: strength-metrics (including a novel metric that I introduce 
here - the proportional partial coefficient), confidence-metrics, and variance-metrics. Therefore, I was able to compare both the specific metrics and the broader theoretical approaches to measuring relative importance, which goes beyond previous comparisons of relative importance metrics that only considered variance-metrics (Murray and Conner 2009). I have outlined a framework to categorise relative importance metrics; and, based on my simulation results and this framework, I have provided recommendations on which metrics are most suitable for use in conservation and ecology.

\subsection{Methods:}

\section{Metrics}

I compared 11 statistical metrics: 4 strength-metrics, 5 variance-metrics, and 2 confidence-metrics (Table 4.1). I included all eight metrics identified in the review outlined in the introduction, plus three additional metrics, which have been suggested in the statistical literature (Grace and Bollen 2005) or used in other disciplines (Healy 1990, Gromping 2007).

\section{Strength-metrics}

All of the strength-metrics are regression coefficients; therefore, to be comparable among predictors that are measured in different units, they must be transformed into a common or comparable scale. In effect, there are three metrics - simple, partial, and averaged partial coefficients - each of which can be re-scaled using one of two transformations, for a total of six possible strength-metrics. I have presented results for only four of these possible six metrics (see below). Regression coefficients can be rescaled using either: a z-score transformation (usually referred to as standardized partial coefficients), which expresses the coefficients in units of one standard deviation (Neter et al. 1990); or a proportional transformation (i.e., proportional partial coefficient), which is 
a novel transformation that re-scales the predictors and the coefficients to a proportional scale, i.e., range from $0-1.0$ and represent a proportion of the range encompassed by the theoretical or practical limits to managing the predictor (conceptually suggested by Grace and Bollen 2005). I have presented results for the partial regression coefficients using both transformations (standardized and proportional partial coefficients, Table 4.1). For simple and averaged partial coefficients, I have only included results using the proportional scale transformation, because the results for the two transformations were similar for all but one criterion; and in this case, the contrast between the transformations was clearly demonstrated by comparing the partial coefficients. For all strength metrics, once they have been transformed to a common scale, the interpretation of relative importance is made by comparing the absolute value for each predictor; predictors with larger strength-metric estimates are more important that those with smaller values (Table 4.1).

The simple regression coefficient, for variable $\mathrm{x}_{1}$, is the slope of a simple linear regression of $\mathrm{y}$ on $\mathrm{x}_{1}$ (hereafter, simple coefficient, Table 4.1). It describes the observed change in $\mathrm{y}$ for a one-unit change in $\mathrm{x}$, while ignoring all other factors. In ecological studies, it is often used as a type of filter, to reduce the number of predictors in a multiple regression by removing those with simple coefficients near zero.

The partial regression coefficient, re-scaled using a proportional scale (hereafter, proportional partial coefficient, Table 4.1), is the slope parameter associated with each $\mathrm{x}$ variable in the multiple regression equation, after the $\mathrm{x}$-variables have been transformed to a proportion of the range of theoretical or practical limits to management. Because a one-unit change in a proportional predictor represents the full range of the predictor, 
proportional partial coefficients represent the expected change in y across the full range of $\mathrm{x}_{1}$, while holding constant all other $\mathrm{x}$-variables in the model. In my simulations, I set the management range limits to $0-20$ for variables $x_{1}, x_{2}$, and $x_{4}$ and to $0-4$ for variable $\mathrm{x}_{3}$. These limits are entirely arbitrary but similar in relation to the scale of each variable (see below in "variables" section).

The partial regression coefficient, re-scaled using a z-score transformation (hereafter, standardized partial coefficient, Table 4.1), represents the change in y for a one-standard deviation change in $\mathrm{x}_{1}$, while holding constant all other $\mathrm{x}$-variables in the model. This is likely the most common approach to re-scaling regression coefficients in ecology (22\% of the studies in my review) and in many other disciplines (Neter et al. 1990).

The averaged partial coefficient (Table 4.1) is calculated as the partial regression coefficient for $\mathrm{x}_{1}$, averaged across all supported models (e.g., all models for which Akaike weight $>0.05$ or $\triangle \mathrm{AIC}<4$ ), and weighted by the Akaike weight (an estimate of the support for each model in the data, Burnham and Anderson 2002). Because, I have expressed these coefficients on a proportional scale, they are effectively identical to the proportional partial coefficients (above) in their interpretation, i.e., the change in y across the full range of $x_{1}$. The two metrics differ in that the inference from the averaged partial coefficients applies to all of the candidate models tested (Burnham and Anderson 2002, i.e., in my simulations, the full model and all of the reduced models that are subsets of it).

\section{Variance-metrics}

All variance-metrics range from $0-1.0$ (sometimes expressed as a percent) and represent a proportion of the total variance in the response, or more commonly, a proportion of the full model $\mathrm{R}^{2}$ that can be allocated to each predictor. Predictors with 
variance-metric estimates closer to 1.0 are considered more important than those with estimates closer to 0 . Because I controlled the overall variance explained by all predictors in my simulations, I have transformed all of the variance metrics (including the simple variance, below) to represent the proportion of the full model $\mathrm{R}^{2}$ (i.e., the "correct" model that includes $\mathrm{x}_{1 . . .3}$ ).

The squared, simple correlation (hereafter, simple variance, Table 4.1) is calculated as the square of the simple correlation between $\mathrm{y}$ and $\mathrm{x}_{1}$. It provides a measure of the proportion of variation in $\mathrm{y}$ that can be explained by $\mathrm{x}_{1}$, while ignoring all other $\mathrm{x}$ variables (referred to as the "zero-order correlation" in Murray and Conner 2009). This metric combines characteristics of the simple coefficient - it ignores all other $\mathrm{x}$-variables - and the standardized partial coefficient - it uses the same z-score standardization to compare all variables on the same scale (both the $\mathrm{x}$-variables and $\mathrm{y}$ are centered to a mean of 0 and standard deviation of 1). Although this metric is normally expressed as a proportion of the variation in the response (Murray and Connor 2009), because I controlled the variation in the response in my simulations and I was only interested in measures of relative importance, I expressed the simple variance as a proportion of the variance explained by the full model (see above), to facilitate comparison with the other variance-metrics.

The classical independent variance partition for $\mathrm{x}_{1}$ (hereafter, the classical independent variance - Table 4.1) is calculated as the change in the variance explained after removing $\mathrm{x}_{1}$ from a regression model that includes all other $\mathrm{x}$-variables. For each $\mathrm{x}$, it represents the explanatory power that can be attributed to it, above and beyond all other $\mathrm{X}$-variables (Legendre and Legendre 1998). When predictors are perfectly orthogonal, the 
classical independent variance for each predictor sums to the $\mathrm{R}^{2}$ for the full model; however, when the predictors are not orthogonal, their sum no longer equals $\mathrm{R}^{2}$ and depending on the presence of suppressor effects, it may be more or less than the full model $\mathrm{R}^{2}$ (Legendre and Legendre 1998, Smith et al. 2009).

The product measure (Pratt 1987) is calculated for $\mathrm{x}_{1}$ by multiplying the coefficient from a regression of $\mathrm{y}$ on $\mathrm{x}_{1}$ by the partial coefficient for $\mathrm{x}_{1}$ of a regression of $\mathrm{y}$ on all $\mathrm{x}$-variables, after standardizing ( $\mathrm{z}$-score) all $\mathrm{x}$ and $\mathrm{y}$ variables. The sum of this measure for all predictors will equal the $\mathrm{R}^{2}$ for the full model. Therefore, the metric can be interpreted as a decomposition of the explanatory power of the full model into partitions that can be attributed to each $\mathrm{x}$-variable (Thomas et al. 1998).

The averaged independent variance partition for $\mathrm{x}_{1}$ is the classical independent variance partition, averaged across all possible models for a given set of predictors. This approach has numerous names in the statistical literature (Gromping 2007) but in conservation and ecological literature it is best known as hierarchical variance partitioning (hereafter, HVP, Table 4.1, Chevan and Sutherland 1991). This metric also sums across all predictors to the full model $R^{2}$ and because it is averaged across all possible models, it is considered to be less conditional on a particular model structure and only conditional on a particular set of predictors (MacNally 2000).

Weighted average independent variance partitions (hereafter, "PMVD", which stands for Proportional Marginal Variance Decomposition, Table 4.1, Feldman unpublished, cited in Gromping 2007) are the same as the averaged partitions in HVP estimates but the averages are weighted. The weights depend on both the explanatory power of the sub-model and the order of the predictors in the model. The weight for a 
particular sub-model will be large if the sub-model has a relatively large $R^{2}$ and predictors that are earlier in the sequential ordering account for a large proportion of the $\mathrm{R}^{2}$. PMVD estimates have the same interpretation as HVP estimates but are considered superior because they are less sensitive to collinearity and the inclusion of spurious predictors (Gromping 2007).

\section{Confidence-metrics}

The probability of the data given that the null hypothesis is true (hereafter, $\mathrm{p}$ value, Table 4.1$)$ is frequently used as an estimate of relative importance ( $34 \%$ of the studies I reviewed), although usually in a less explicit or quantitative way than the other metrics I compared. For example, variables with p-values $>0.05$ are usually excluded from modelling - so, effectively considered unimportant - and variables with very low pvalues are considered more important than those with p-values closer to 0.05 (Johnson 1999). Formally, this metric represents the probability of sampling data with parameter values this large (or larger), given that the null hypothesis - usually that the coefficient is zero - is true. Informally and incorrectly, p-values are often treated as an estimate of the probability that the coefficient is zero, and therefore, that a particular variable can be ignored (Stephens et al. 2007).

The summed Akaike weight for $\mathrm{x}_{1}$ is calculated by adding the Akaike weights for all of the supported models that include $\mathrm{x}_{1}$ (Burnham and Anderson 2002). These summed weights are interpreted as a quantitative estimate of the evidence that each $\mathrm{x}$ variable is important (sensu Burnham and Anderson 2002, i.e., that, conditional on the data, it should be included in a parsimonious model). Summed Akaike weights range from $0-1.0$ and predictors with values closer to 1.0 are considered more important. 


\section{Simulation methods}

The simulations were conducted in R statistical software ( $\mathrm{R}$ Development Core Team 2009). The R-package "relaimpo" was used to calculate three of the variance metrics: HVP, PMVD, and the product measure. The R-package "MuMIn" (available online at: http://r-forge.r-project.org/projects/mumin/) was used to calculate the two multi-model inference metrics: summed Akaike weights.

\section{Variables}

For each simulation, I created a matrix of predictor variables and response variables to represent my population, with five columns and 100000 rows - one column for the response and each of four predictor variables $\left(y, x_{1}, x_{2}, x_{3}\right.$, and, for the spurious predictors criterion, $\mathrm{x}_{4}$ ). Three of the $\mathrm{x}$-variables in each matrix $-\mathrm{x}_{1}, \mathrm{x}_{2}$, and $\mathrm{x}_{4}-$ were random, normally distributed variables (mean $=12.5$, standard deviation $=2.5)$. The remaining variable $-\mathrm{x}_{3}-$ was also a random, normally distributed variable (mean $=2.5$, standard deviation $=0.5$ ), which represented a predictor with a similarly shaped distribution but in different units than the other predictors. Further details on the $\mathrm{X}$ matrices are below in the criterion-specific sections.

The $y$ (response) variables associated with each of the simulations were created using the following linear equation:

$$
y=\beta_{1} * x_{1}+\beta_{2} * x_{2}+\beta_{3} * x_{3}+\varepsilon \quad \text { Equation } 1 .
$$

Where $\beta_{1 \ldots 3}$ represented the true functional influence of variable $x_{1 \ldots 3}$ on the response and $\varepsilon$ was a random, normally-distributed error variable. This error term had a mean of 0 and a variance scaled for each set of simulations, such that a linear regression of $y$ on all of the predictors would explain approximately $50 \%$ of the variation in $y$. 
Unless otherwise indicated, the true functional effects of each $\mathrm{x}$-variable in all of the simulations were:

$$
\mathrm{y}=1.0 * \mathrm{x}_{1}+0.5 * \mathrm{x}_{2}+0.75^{*} \mathrm{x}_{3}+\varepsilon \quad \text { Equation } 2 .
$$

When expressed on a common scale, the relative strengths of their true effects are: $\beta_{1}{ }^{\prime}=1.0, \beta_{2}{ }^{\prime}=0.5$, and $\beta_{3}{ }^{\prime}=0.15$; because the units of $\mathrm{x}_{3}$ are 5 times as large in relation to its mean and standard deviation, as they are for $\mathrm{x}_{1}$ and $\mathrm{x}_{2}$.

For each of 20 levels of each criterion (e.g., 20 different sample sizes), I drew a random sample from the relevant population and calculated each metric for $\mathrm{x}_{1}, \mathrm{x}_{2}$, and $\mathrm{x}_{3}$ $\left(\mathrm{x}_{1}, \mathrm{x}_{2}, \mathrm{x}_{3}\right.$, and $\mathrm{x}_{4}$ for the spurious predictor criterion). I iterated this sampling and calculation procedure 1000 times at each level of the criterion, which generated a distribution of 1000 estimates for each metric at each of the 20 levels of the criterion. I then plotted the mean of each metric against the criterion values (e.g., sample size on the $\mathrm{x}$-axis).

\section{Criteria:}

Sample Size

To compare each metric's dependence on sample size, I simulated a population where the $\mathrm{x}$-variables were perfectly orthogonal (all pairwise $\mathrm{r}=0.0$ ). I then drew random samples from this population using 20 different sample sizes (ranging from $n=30$ to $n=$ 1000).

\section{Collinearity}

To compare each metric's dependence on collinearity among predictors, I simulated 20 populations, where each population had a different level of collinearity among the $\mathrm{x}$-variables. All pairwise correlations among $\mathrm{x}_{1 \ldots 3}$ were the same in each matrix and ranged from 0 in the least collinear matrix to 0.99 in the most collinear matrix. 
I then randomly selected 500 rows from each matrix and calculated the metrics for each $\mathrm{x}$ using the $\mathrm{n}=500$ sample.

Relative Variation

To compare each metric's dependence on changes in the range of variation sampled for each predictor, I used a simulated population with orthogonal x-variables. I then drew samples of 500 from this population, which were random except that the range of potential $\mathrm{x}_{2}$ values sampled was successively narrowed, in 20 steps, while maintaining the full range and variance in $x_{1}$ and $x_{3}$. Across the 20 levels of this criterion, the range of the $x_{2}$ variable decreased from $100 \%$ to $2.5 \%$ of its original range.

Spurious predictors

To compare how sensitive each metric was to the inclusion of a spurious predictor, I created $20 \mathrm{XY}$ matrices representing populations where the $\mathrm{x}_{1 \ldots 3}$ were orthogonal but the correlation between $\mathrm{x}_{1}$ and $\mathrm{x}_{4}$ increased across the populations from $0-0.99$. Although the pairwise correlation of $\mathrm{x}_{4}$ with $\mathrm{y}$ increased along with its increasing correlation with $\mathrm{x}_{1}$, its true influence was always 0 because it was never included in equation 1 . The true effects of $x_{1 \ldots 3}$ were the same as for the previous tests. I used the same sampling, analysis, and plotting process as in the previous criteria only in this case, I included the spurious $\mathrm{x}_{4}$ variable along with all of the influential $\mathrm{x}_{1 \ldots 3}$ variables in the calculations for each metric.

Suppressor effects

Finally, to test for biases in the presence of suppressor effects, I simulated the same 20 populations created to test for sensitivity to collinearity; however, in this case, $x_{2}$ had the same absolute influence as $\mathrm{x}_{1}$ but in the opposite direction:

$$
\mathrm{y}=1.0 * \mathrm{x}_{1}-1.0 * \mathrm{x}_{2}+0.75 * \mathrm{x}_{3}+\varepsilon \quad \text { Equation } 3
$$


Again, I did the same sampling, analysis, and plotting process as for the previous criteria.

\section{Interpreting the plotted results}

To simplify the interpretation of the simulation output, I arranged the figures so that plots of metrics that satisfied a particular criterion would appear similar. I considered a metric to have satisfied each criterion if the metric: 1) gave distinct estimates of importance for all predictors, i.e., non-overlapping lines representing the average estimated value for each predictor; 2) ranked the predictors according to their true relative influence on the response, i.e., $\mathrm{x}_{1}>\mathrm{x}_{2}>\mathrm{x}_{3}$ except for the suppressor criterion where $\left|x_{1}\right|=\left|x_{2}\right|>x_{3}$; and 3) remained constant across the range of simulated conditions, i.e., horizontal lines across all or most of the range of the $\mathrm{x}$-axes. I also inverted the $\mathrm{x}$-axes for the sample size and relative variation criteria, so that the extreme left of all $\mathrm{x}$-axes represented the least severe of the simulated conditions (e.g., the largest sample size and the largest range of variation). Therefore, the left side of each plot also most accurately reflected the true effects of each $\mathrm{x}$-variable for all metrics. In addition, I inverted the $\mathrm{y}$ axes for all plots of p-values, so that x-variables that were similarly "important" according to each metric were placed in similar locations with respect to the top and bottom of the plots.

\subsection{Results}

The only estimates of relative importance that satisfied all 5 criteria were the proportional partial coefficients and averaged partial coefficients, which are both strength-metrics and are expressed on the same proportional scale (Figures $4.1-4.5$ ). The standardized partial coefficients satisfied 4 of the 5 criteria (Figures 4.1, 4.2, 4.4, and 4.5) but were linearly dependent on the relative variation sampled in $x_{2}$ (Figure 4.3). 
Simple coefficients failed to satisfy 3 of 5 criteria; they depended on the level of collinearity, the inclusion of a spurious predictor, and the strength of suppressor effects (Figures 4.2, 4.4, and 4.5 respectively).

The variance-metrics were all independent of sample size (Figure 4.1). For most other criteria, variance-metrics were highly dependent on the simulated conditions (Figures 4.2, 4.3 and 4.4) and in at least one case, the dependencies were complex and unintuitive (i.e., Figure 4.5). One variance-metric was independent of collinearity for most of the range of simulated values (PMVD in Figure 4.2), and two were independent of the strength of correlation with a spurious predictor (product measure and PMVD in Figure 4.4). Overall, the best variance metric (PMVD) satisfied only 3 of the 5 criteria and most satisfied only 1 .

The confidence-metrics failed to satisfy any of the 5 criteria (Figures 4.1-4.5). They often failed to distinguish among or correctly rank all of the predictors (e.g., estimates for $\mathrm{x}_{1}$ and $\mathrm{x}_{2}$, and in some cases $\mathrm{x}_{3}$, overlap in most plots, Figures 4.1-4.5), they depended strongly on the characteristics of the sample (Figures $4.1-4.3$ ) and they were biased by the inclusion of a spurious predictor, and suppressor effects (Figures 4.4 and $4.5)$.

\subsection{Discussion}

The success of many of the strength-metrics and the failures of variance and confidence-metrics can be explained by considering relative importance metrics within a clear conceptual framework (Figure 6). The three categories of metrics are connected through two fundamental properties of statistical analyses in ecology: biological significance and statistical significance. Strength-metrics of relative importance are 
primarily measures of biological significance, which estimate a property of the statistical population (i.e., the parameter values in the linear equation that is assumed to represent the underlying ecological phenomenon in all regression modelling). Confidence-metrics are primarily measures of statistical significance, which describe properties of the statistical sample (i.e., the probability of the data, given the parameter values; or the support in the data for the parameter values). Variance-metrics are influenced by both statistical and biological significance (i.e., statistically, the sample and the population) because they are calculated with measures of model fit (explained variance or deviance), which depend on both the error around a parameter estimate and the true parameter value.

All three types of metrics are valuable measures in some contexts, given certain assumptions; however, not all metrics support the type of inference that is required to measure the relative benefit of competing conservation options. Metrics fail to meet my criteria, not because the metrics are flawed, but because my criteria make it clear that comparing the relative potential benefits to conservation requires a particular type of inference. Comparisons of relative importance in conservation require predicting the effects of manipulating the system. If confounding factors have been controlled and the predictors in the regression reasonably represent ecological factors that can be independently managed, to some degree, and that are thought to have a causal effect on the response (James and McCulloch 1990), then partial coefficients provide this required prediction. Confidence-metrics compliment or qualify those predictions but do not represent the effects themselves. Variance-metrics provide valid descriptions of the existing conditions (assuming appropriate random sampling) but they do not provide clear implications for manipulating the existing conditions. 
Of the strength-metrics, proportional partial coefficients and the averaged partial coefficients on a proportional scale are the best estimates of the likely effects of independently managing competing conservation options. They are the clearest measure of biological significance; they are independent of the sample characteristics; and, they are not biased by spurious predictors or suppressor effects. They also have an intuitive and clear interpretation; they represent a direct estimáte of the predicted benefit to conservation from managing the environmental factor represented by each predictor. By re-scaling each predictor from $0-1.0$, which represent, respectively, the minimum and maximum values that can be reached through management, these coefficients represent the total average change in the response from manipulating each predictor. An additional benefit to re-scaling predictors or coefficients on a proportional scale is that the choice of the practical or theoretical limits requires an explicit discussion of what might be possible under any practical or theoretical limitations. Individual studies can calculate proportional coefficients for multiple practical ranges and thereby analyse the sensitivity of their relative importance conclusions to the choice of these limits. In addition, published estimates of proportional partial coefficients can be transformed to different limits and thereby, be easily and transparently compared across studies that used different ranges. Although my simulations showed similar patterns for these coefficients whether they came from a single model or were averaged across all supported models, it is important to note that these two approaches are far from identical on both mathematical and philosophical grounds (Burnham and Anderson 2002). Indeed, because averaged proportional partial coefficients are weighted by a confidence-metric - the Akaike weight - in some conditions, they may be prone to similar limitations as the variance-metrics 
(e.g., note the similarities between the results for averaged partial coefficients and the variance-metrics in Figure 1).

I have used a proportional re-scaling that represents the full range of management possibilities but it is important to note that any data-independent re-scaling of a partial regression coefficient will have the same properties as my proportional partial coefficients. There may be situations where scaling with respect to the full range is inappropriate. For example, if some of the correlation between predictors is causal, then it is not possible to manipulate one variable completely independently of the other. In these cases, re-scaling to a smaller range (e.g., $10 \%$ of the range of management limits) may make the direct interpretation of the coefficients more appropriate. Similarly, in some cases coefficients re-scaled to their conditional variation may be more appropriate (as suggested in Grace and Bollen 2005).

Standardized partial coefficients and simple coefficients are less useful than the other two strength metrics because they combine measures of statistical and biological significance (Figure 6). Standardized partial coefficients are re-scaled to a property of the statistical sample - the standard deviation of each predictor in that particular study. Therefore, the $\mathrm{z}$-score transformation turns a sample-independent measure, the partial regression coefficient, into a sample-dependent measure. In contrast, the proportional transformation re-scales to a pre-determined range that is independent of the sample. Simple coefficients are biased and sample dependent because they are sensitive to collinearity. Predictors are rarely, if ever, perfectly orthogonal in empirical studies; and, studying the relative importance of management options implies manipulating the factors independently, i.e., changing the correlations among them. Therefore, simple coefficients 
are almost always biased estimates of effect strength. Limiting correlations among predictors to $<0.7$ or another arbitrary threshold, which is common in ecological studies, will not remove these biases because they show no such thresholds.

Although variance metrics dominate the literature on relative importance both in ecology (MacNally 2000, Murray and Conner 2009, 44\% of the studies in my review) and other disciplines (Gromping 2007, Johnson and LeBreton 2004), my simulations show they are of limited use in conservation because they combine measures of biological and statistical significance (Figure 6) and cannot be compared across studies with different sample characteristics. This merging of sample and population properties also means variance-metrics cannot distinguish between a strong but uncertain effect and a certain but weak effect, which is an important distinction in a management context. Variance-metrics have been popular, likely because they describe how the $R^{2}$ of a full model might be partitioned among the predictors, and these partitions have been interpreted as measures of each predictor's "explanatory power" or "explanatory value" (MacNally 2000, Gromping 2007). However, this descriptive partitioning only applies to random sample of an existing population and does not clearly imply the effects of manipulating that population. Previous recommendations for measuring relative importance in conservation recommended the use of two variance-metrics: HVP estimates and simple variance (referred to as "independent effects" and "zero-order correlations" respectively in Murray and Conner 2009); however, these metrics performed poorly in my simulations and met only one of my five criteria.

Even the variance-metrics that performed the best - the product measure and PMVD estimates - met only two or three of my criteria. The product measure is not 
biased by spurious predictors because it is always very low for any predictor whose partial coefficient is approximately zero. However, it is biased by suppressor effects and collinearity and it is sample-dependent for the same reasons as all other variance-metrics: it is sensitive to the sampled variance of each predictor. PMVD estimates were created as a modification of the HVP approach that would be less sensitive to collinearity (Gromping 2007). My results show that they are unbiased by spurious predictors and much less sensitive to collinearity than HVP estimates; however, they are biased by suppressor effects - they underestimate the importance of the predictor that has more suppressor relationships with other predictors (i.e., $\mathrm{x}^{2}$ in my simulations). Therefore, independence from collinearity for PMVD estimates can only be assumed if there are no conflicting effects among predictors (e.g., only positive correlations among predictors and the effects for all predictors are in the same direction; see Smith et al. 2009 for a more detailed description of these suppressor effects).

Not surprisingly (e.g., Kruskal and Majors 1989, Achen 1982), my simulations showed that confidence-metrics do not satisfy the criteria for measures of relative importance. Confidence-metrics answer questions about statistical significance (Figure 6) and they are only appropriate given a random sample of a well-defined population. They do not answer questions about how much benefit to expect from a particular conservation action. Nor do they provide information that is comparable across studies with differences in sample size, collinearity, or relative sampled variation in the predictors. However, they can provide important information on whether an assessment of relative importance might be due to chance and how confident or certain it is (Yoccoz 1991, 
Johnson 1999). To avoid confusion, this type of evidence is probably better referred to as "relative confidence" or "relative certainty", and not relative importance.

Summed Akaike weights and p-values are useful measures in some situations, but not all (Johnson 1995, Stephens et al. 2005). Interestingly, despite having very different philosophical and mathematical underpinnings, p-values and summed Akaike weights showed almost identical patterns of variation in all of my simulations. Their functional similarity is a further reminder (Stephens et al. 2007) that information theoretic methods are susceptible to many of the same inferential pitfalls as null-hypothesis testing. Finally, although Idid not use them in any of my simulations because they are not directly interpreted as measures of relative importance, confidence intervals provide more information than p-values or summed Akaike weights (Johnson 1999 and Burnham and Anderson 2002) and therefore, they are likely more useful measures of relative confidence.

Proportional partial regression coefficients outperformed all other metrics in my simulations but it is important to remember that their usefulness in any real-world situation strongly depends on the assumption that the underlying model is a satisfactory representation of the ecological processes (Pedhazur 1997, James and McCulloch 1990). This qualification does not affect my comparisons among metrics, because it is common to all of the methods Icompared; however, careful consideration of the model is critical. For example, there may be practical or theoretical limitations to the appropriateness of the underlying model; if the possible range of correlations between two predictors is constrained by some theoretical or practical limitation, then the range used to re-scale the predictors must reflect these practical limitations (Grace and Bollen 2005). Predicting the 
effects of manipulating an ecological system may also involve extrapolating a statistical model beyond the range of the data. For example in a particular study with correlated predictors, if we can reasonably expect to manipulate those predictors outside of the existing range of correlation (i.e., combinations of predictors for which we do not have data), then extrapolating may be appropriate but it relies even more heavily on the assumption that the model reflects reality (Gelman and Hill 2007). In addition, if there are influential factors that are un-modelled and correlated with the predictors, then proportional partial coefficients, and all the other metrics of relative importance, will be biased (Neter et al. 1990, Smith et al. 2009). Finally, the source of the data is particularly important. Data from manipulative experiments are in many cases superior to those from pseudo-experimental correlative studies for drawing inferences about management or conservation actions (James and McCulloch 1990). Ultimately, none of the metrics are immune to the fact that the inferential strength of any study measuring relative importance will always depend on the assumptions of the underlying model. My point is that for any given study and set of model assumptions, proportional partial coefficients are the most useful measures in a conservation context.

\section{Recommendations for estimating relative importance in conservation}

If ecologists and conservation scientists want their work to be useful for and relevant to policy and applied conservation, then they must provide results that are easily and clearly interpretable in that context (Pullin and Knight 2009). Proportional partial regression coefficients (or any other partial regression coefficient, on a data-independent scale) provide those results. They are the best measures of relative importance in conservation, among the 11 metrics I compared here. With careful consideration of the data collection and how well the statistical model represents the underlying ecological 
process, they can be interpreted as an estimate of the maximum change in a response from independently manipulating or managing each predictor in a regression analysis. In comparison, variance-metrics are more sample-dependent, less flexible, more biased, and much more difficult to interpret and compare across studies. Proportional partial coefficients must be reported with an associated confidence-metric (e.g., confidence intervals), to estimate the relative confidence in an estimate of relative importance. Finally, the theoretical or practical limits used to re-scale the predictors or coefficients must also be published so that comparisons can be made across studies and under varying theoretical or practical ranges. 


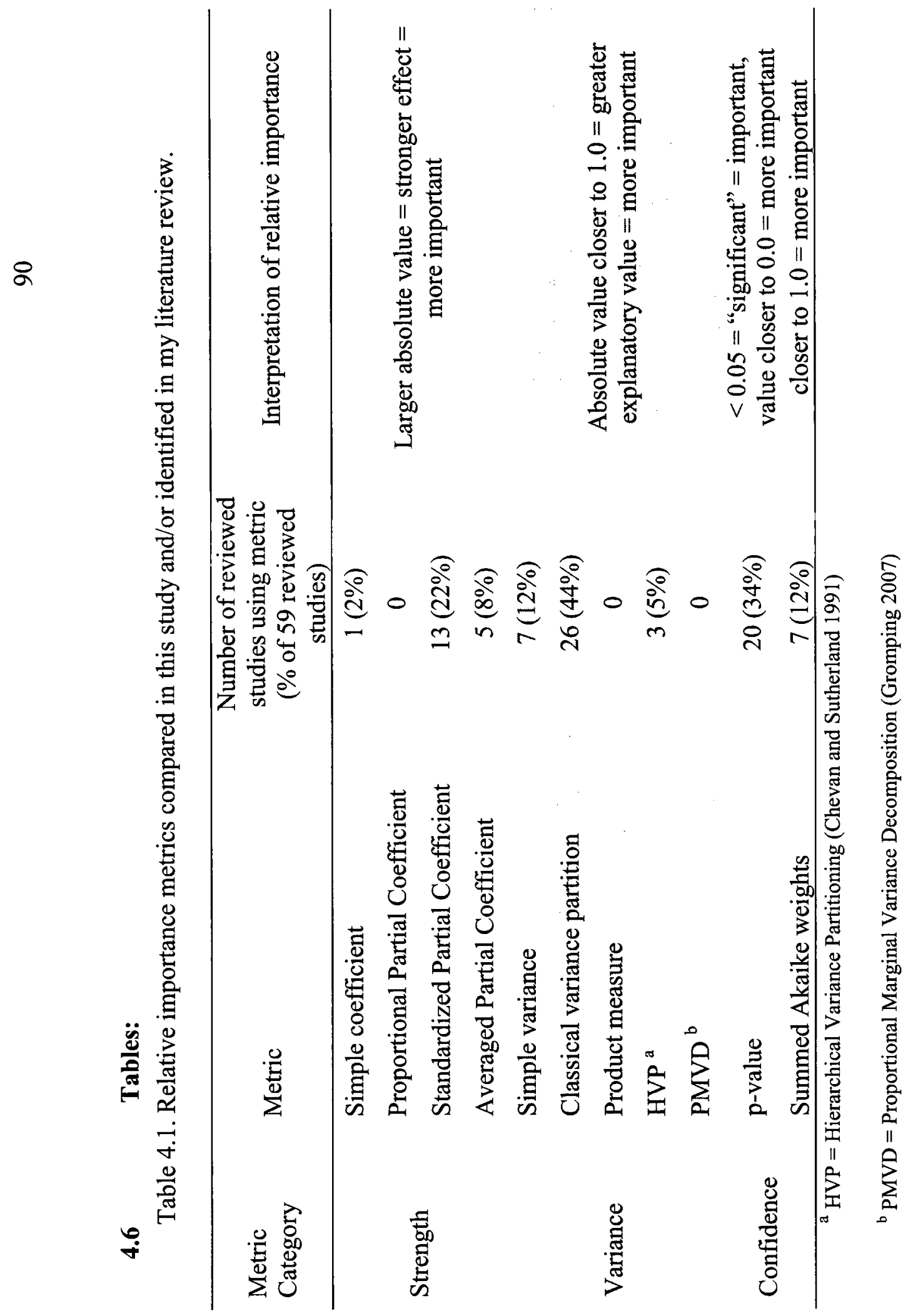




\section{$4.7 \quad$ Figures}
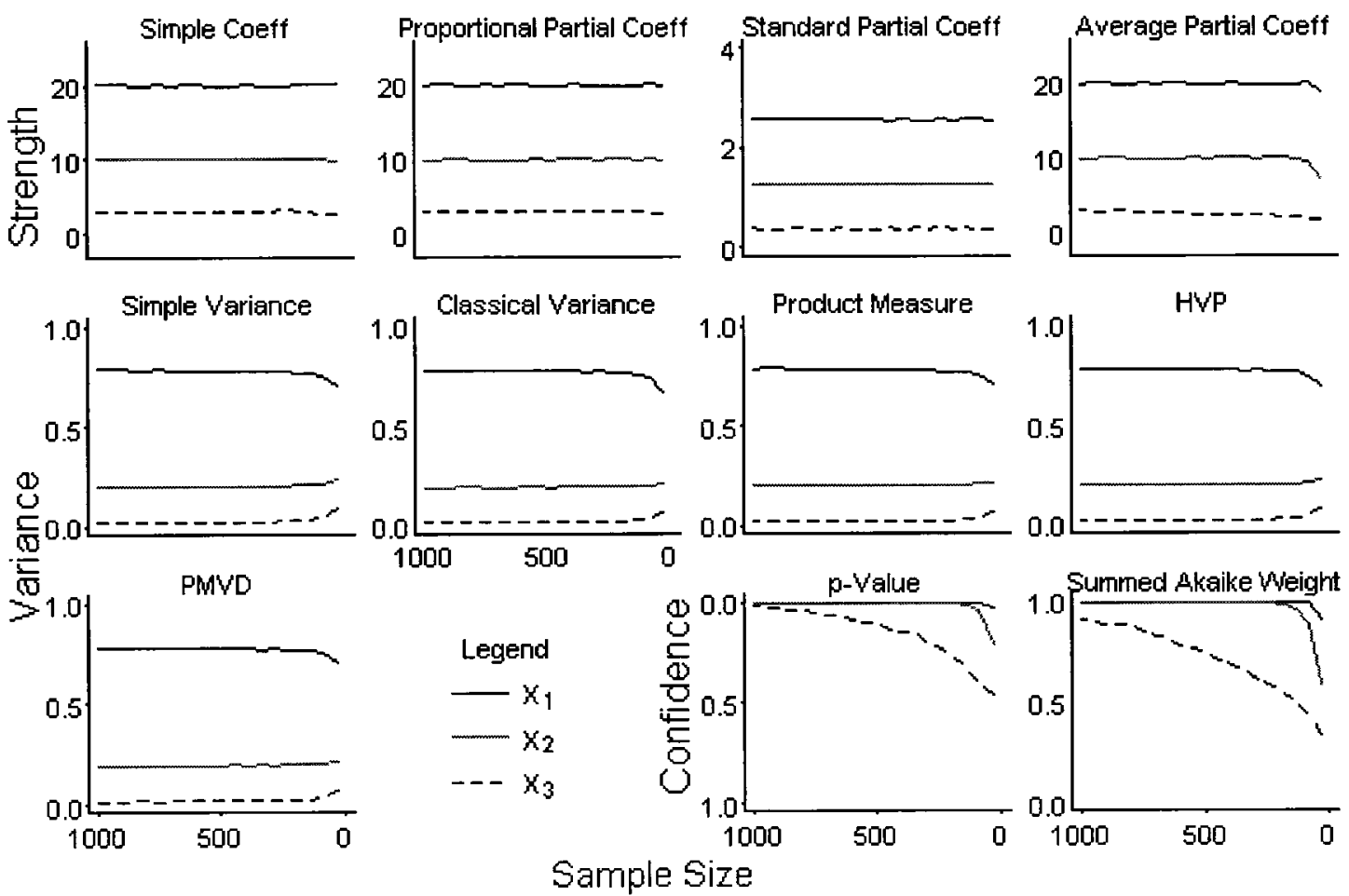

Sample Size

Figure 4.1. Relationships between sample size and strength-, variance-, and confidencemetrics of relative importance, calculated for three orthogonal, simulated predictors with linear effects on a response (Equation 2). Each plot is arranged so that the metrics that satisfy the sample size criterion show: three non overlapping lines, rank $\mathrm{x}_{1}>\mathrm{x}_{2}>\mathrm{x}_{3}$, and are constant from left (larger samples) to right (smaller samples) along the $\mathrm{x}$-axis. Lines represent the mean estimated metric value across 1000 iterations of a random sample, drawn from an $\mathrm{N}=100000$ population of simulated data. 


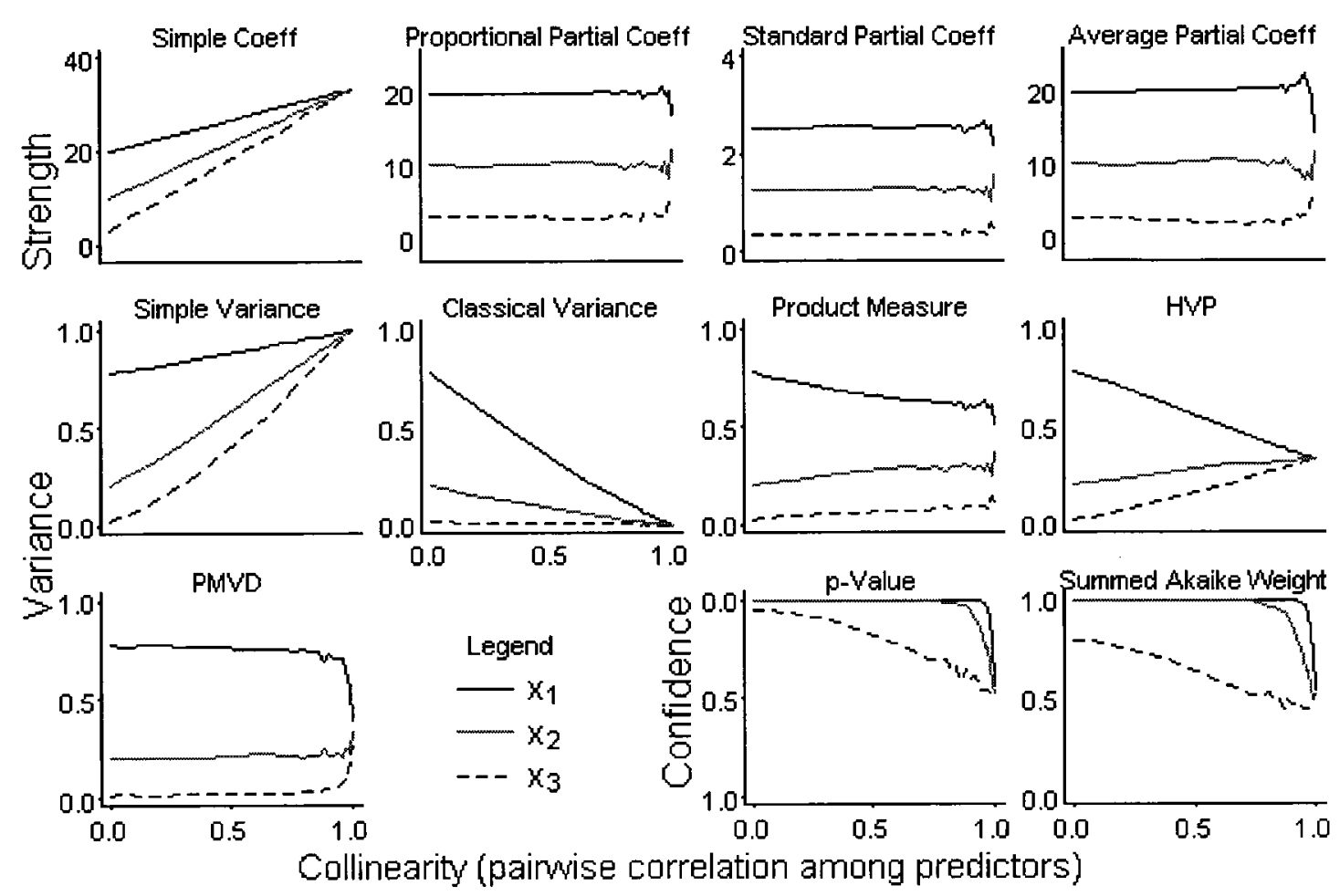

Figure 4.2. Relationships between collinearity among predictors and strength-, variance-, and confidence-metrics of relative importance, calculated for three simulated predictors with linear effects on a response (Equation 2). Plots are arranged so that the metrics that satisfy the collinearity criterion show: three non overlapping lines, rank $\mathrm{x}_{1}>\mathrm{x}_{2}>\mathrm{x}_{3}$, and are constant from left (orthogonal variables) to right (pair-wise correlations $=0.999$ ) along the $\mathrm{x}$-axis. Lines represent the mean estimated metric value for each predictor across 1000 iterations of a random sample of size $n=500$, from an $N=100000$ population of simulated data. 

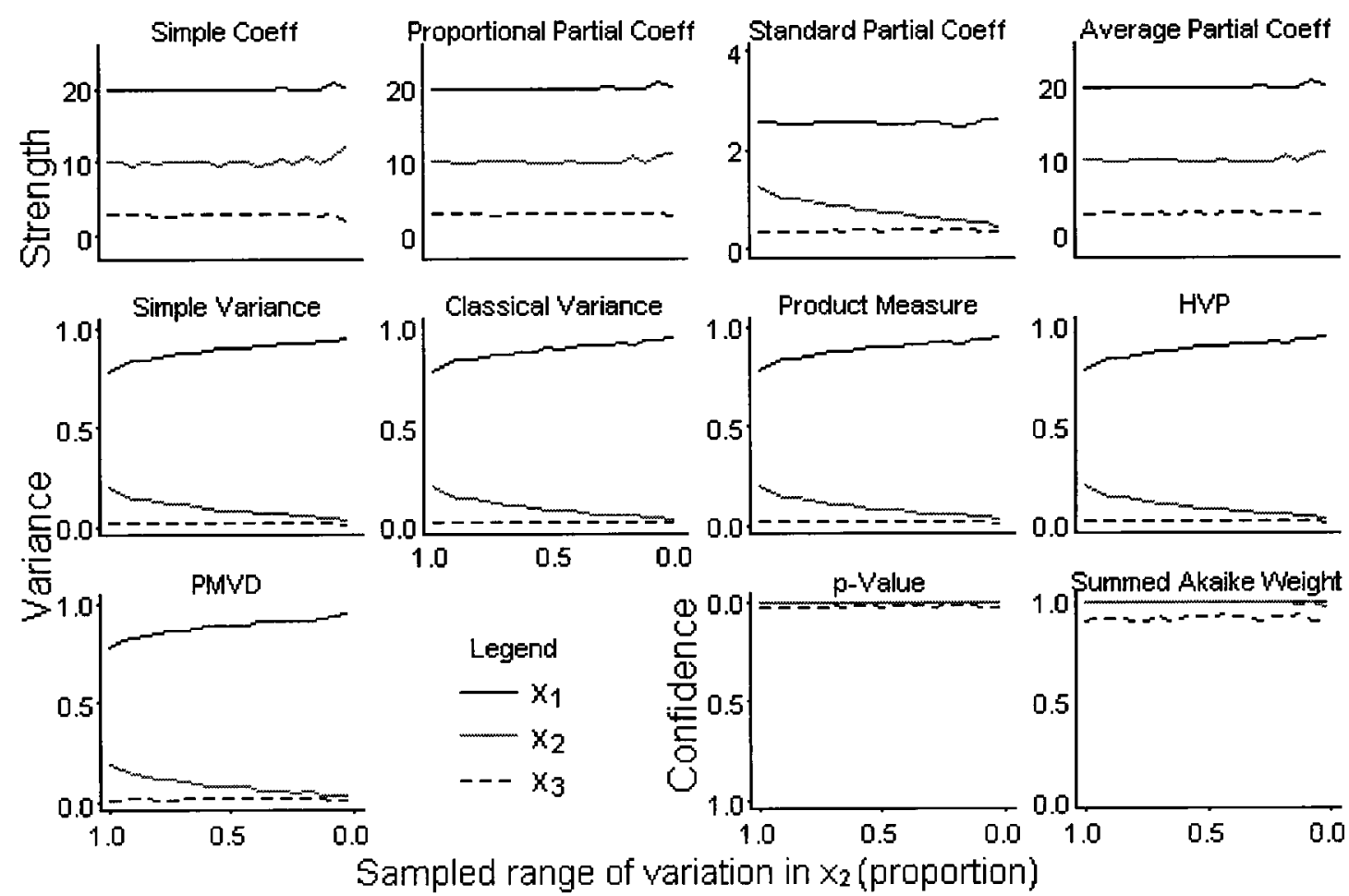

Sampled range of variation in $x_{2}$ (proportion)

Figure 4.3. Relationships between the relative variation (i.e., the proportion of the original range of $x_{2}$ that was sampled) and strength-, variance-, and confidencemetrics of relative importance, calculated for three orthogonal, simulated predictors with linear effects on a response (Equation 2). Each plot is arranged so that the metrics that satisfy the relative variation criterion show: three non overlapping lines, rank $\mathrm{x}_{1}>\mathrm{x}_{2}>\mathrm{x}_{3}$, and are constant from left from left (full range of $\left.x_{2}\right)$ to right $\left(2.5 \%\right.$ of the original range in $x_{2}$ sampled) along the $\mathrm{x}$-axis. Lines represent the mean estimated metric value for each predictor and the upper and lower $95^{\text {th }}$ percentiles, across 1000 iterations of a random sample of size $n=500$, from an $\mathrm{N}=100000$ population of simulated data. 


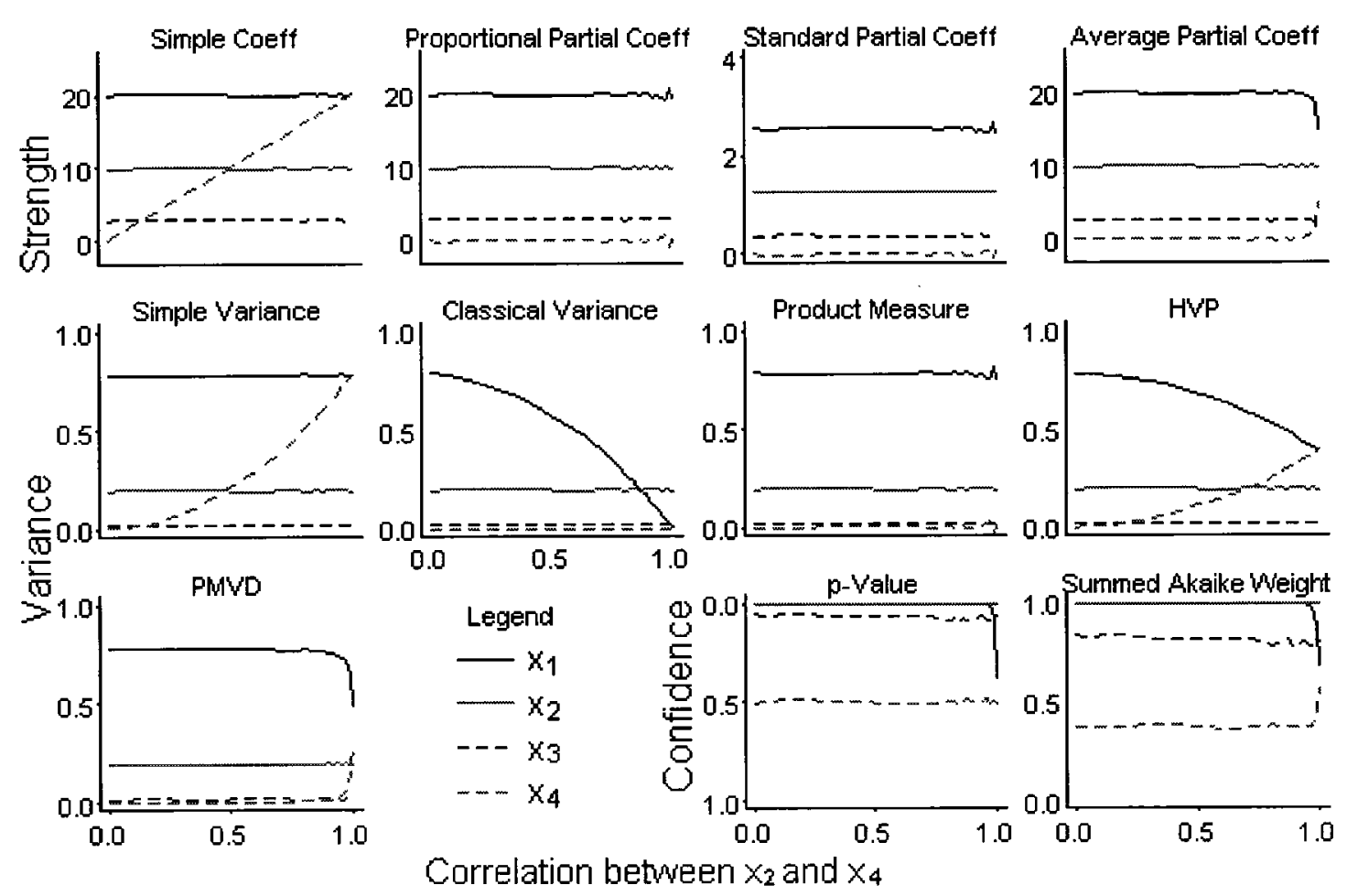

Figure 4.4. Relationships between the correlation of a spurious predictor $\left(\mathrm{x}_{4}\right)$ with an influential variable $\left(\mathrm{x}_{1}\right)$ and strength-, variance-, and confidence-metrics of relative importance, calculated for three simulated predictors with linear effects on a response (Equation 2) and a fourth, spurious predictor with no effect on the response $\left(\mathrm{x}_{4}\right)$. Plots are arranged so that the metrics that satisfy the spurious predictor criterion show: three non overlapping lines, rank $\mathrm{x}_{1}>\mathrm{x}_{2}>\mathrm{x}_{3}>\mathrm{x}_{4}$, and are constant from left (no correlation between $\mathrm{x}_{1}$ and $\mathrm{x}_{4}$ ) to right (pair-wise correlation between $\mathrm{x}_{1}$ and $\mathrm{x}_{4}=0.999$ ) along the $\mathrm{x}$-axis. Lines represent the mean estimated metric value for each predictor across 1000 iterations of a random sample of size $n=500$, from an $\mathrm{N}=100000$ population of simulated data. 

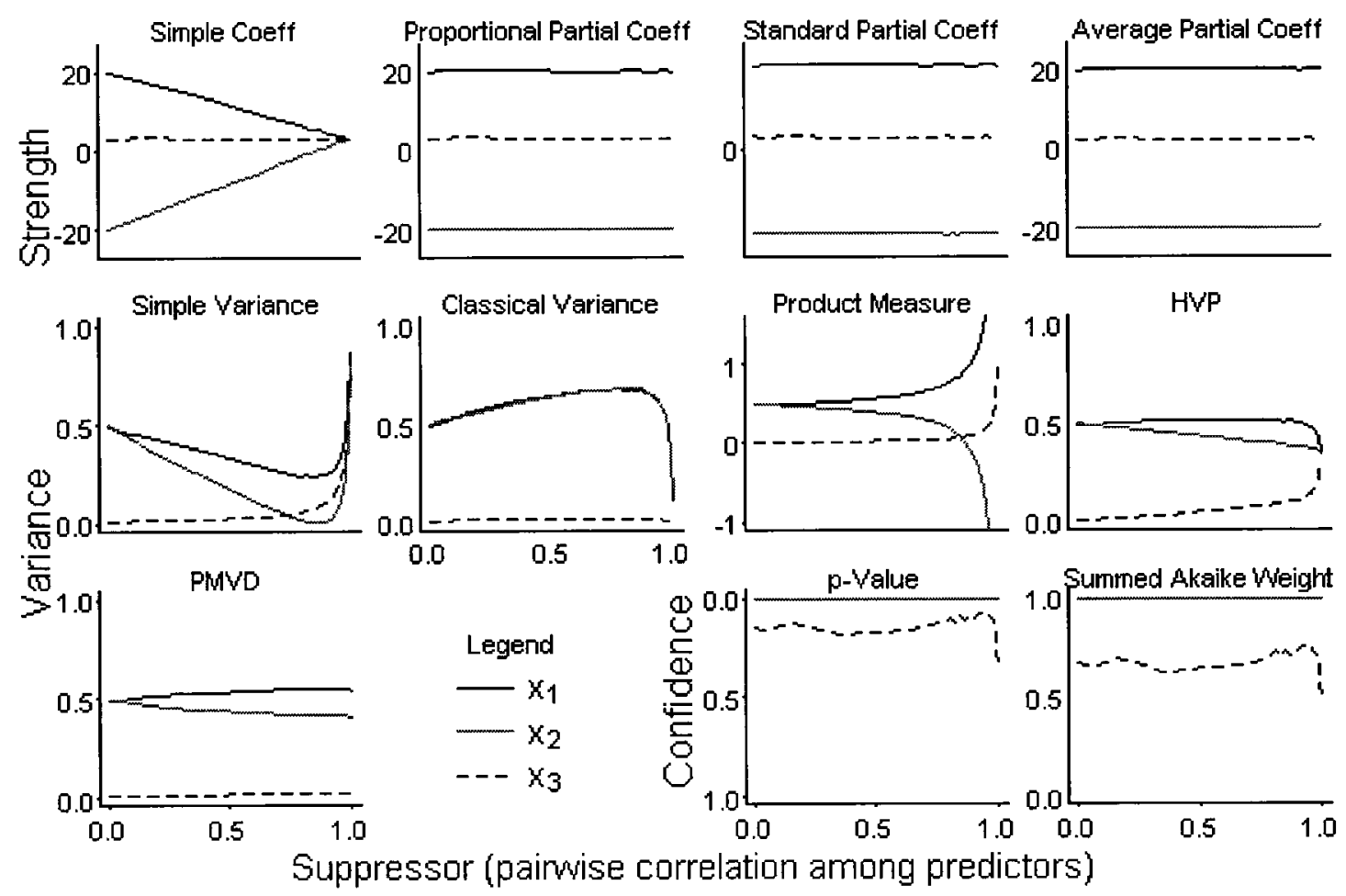

Figure 4.5. Relationships between strength of a suppressor effect and strength-, variance-, and confidence-metrics of relative importance, calculated for three simulated predictors with linear effects on a response (Equation 3). Plots are arranged so that the metrics that satisfy the suppressor predictor criterion show: three non overlapping lines, rank $\left|\mathbf{x}_{1}\right|=\left|\mathrm{x}_{2}\right|>\mathrm{x}_{3}$, and are constant from left (orthogonal variables) to right (pair-wise correlations $=0.999$, where the effects of $x_{1}$ and $x_{2}$ completely cancel each other) along the $\mathrm{x}$-axis. Lines represent the mean estimated metric value for each predictor, across 1000 iterations of a random sample of size $n=500$, from an $N=100000$ population of simulated data. 


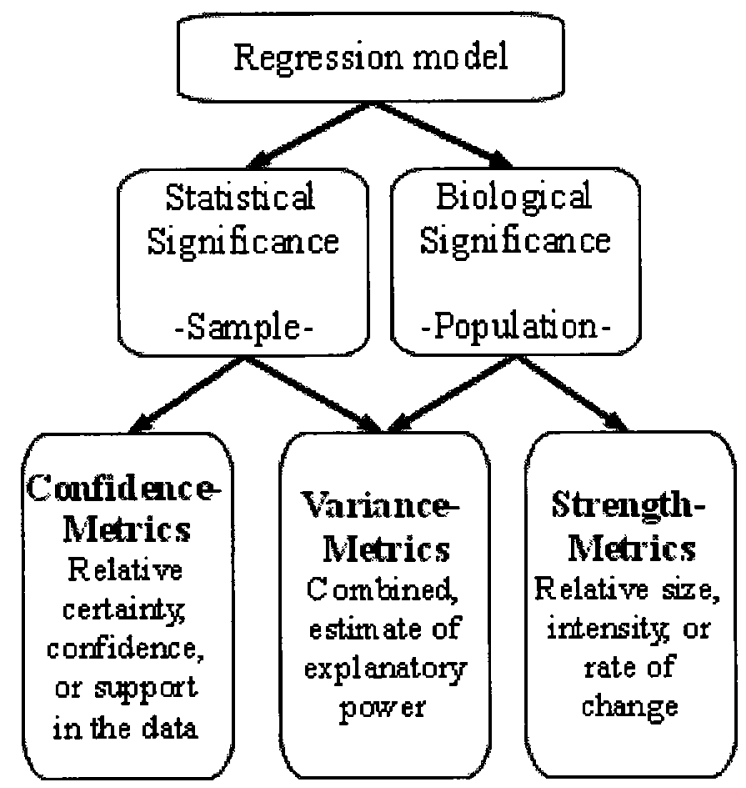

Figure 4.6. Framework showing the underlying connections among three categories of relative importance metrics, which are created by the dichotomy of statistical and biological significance within regression modeling. Strength-metrics measure biological significance, which is a characteristic of the underlying ecological phenomenon or the statistical population. Confidence-metrics measure statistical significance, which is a characteristic of the statistical sample. Variance-metrics measure both statistical and biological significance and are influenced by both the sample and the ecological phenomenon. 


\section{Chapter 5. Residential vegetation can be just as important as parks and greenspace for bird diversity in urban areas.}

\subsection{Chapter Summary:}

Estimating the relative importance of residential vegetation (gardens, yards, and street-trees) and non-residential vegetation (parks and other large greenspaces) is important so that competing options for conservation planning can be prioritized. However, estimating their relative effects is complicated because: the area and structure of the two types of vegetation are often correlated; and their relative effects may be different at local and landscape scales. I used data from an urban breeding-bird monitoring program to compare the relative effects of local and landscape residential vegetation area and structure (percent tree cover) on species richness and an index of conservation value. I then estimated the relative importance of potential manipulations to residential and non-residential vegetation area (i.e., as competing management options), within the practical limits to their management that are imposed by human population density. The local effects of increasing residential and non-residential vegetation area were similar and positive on all measures of species richness and conservation value. Non-residential vegetation had an additional landscape-scale influence on bird diversity that residential vegetation did not. When I considered their relative importance within practical limits to their management, the area of residential vegetation became more important as human population density increased. 


\subsection{Introduction}

Urban areas are much more important for biodiversity conservation than their relatively small physical footprint would suggest. They provide habitat for many species, including species at risk (Evans et al. 2009); and that habitat may be particularly important because urban areas tend to coincide with regions of high biodiversity (Balmford et al. 2001, Deguise and Kerr 2006). Urban areas also have a vital, if indirect, role to play because an increasing proportion of the human population lives in urban areas and positive experiences with native flora and fauna help build an appreciation for local biodiversity and social acceptance of environmental attitudes (Turner et al. 2004, Miller 2005).

The area and structure of vegetation has a strong influence on bird diversity in urban areas (reviewed in Chase and Walsh 2006 and McKinney 2008). Urban areas with a greater area of vegetation have higher species richness (Lancaster and Rees 1979) and fewer non-native species (Blair 1996). In addition, vegetation structure has a strong effect; increased vertical structure (e.g., increased tree cover and a lower proportion of mowed grass) increases species richness and functional diversity (Melles et al. 2003, Clergeau et al. 1998).

Studies of urban biodiversity have identified effects of both residential vegetation and non-residential vegetation but their relative effects are unknown. The majority of studies have focused on the effects of non-residential vegetation (i.e., large areas of natural vegetation such as parks and vacant land) and found positive effects of increasing area and structural complexity (Lancaster and Rees 1979, Clergeau et al. 1998, see review in Chase and Walsh 2006). Some more recent studies have found similar effects 
of residential vegetation (i.e., privately-owned gardens and street-trees, Fernandez-Juricic 2000, Daniels and Kirkpatrick 2006). A few studies have examined both types of vegetation. However, these studies did not compared their relative effects, they either used cumulative measures that combined the effects of residential and non-residential vegetation (e.g., Melles et al. 2003 Evans et al. 2009) or estimated their separate effects on different response variables (e.g., species richness measured either in residential or non-residential areas, Donnelly and Marzluff 2006).

It is important to know the relative effects of manipulating the area and structure of vegetation in either residential or non-residential areas because, although they are often correlated, they can be managed independently. A conservation-based management decision that requires choosing between altering the area of either residential or nonresidential vegetation, must have a way of comparing their relative potential benefits to conservation (Chapter 4, Wilson 2007). However, comparing their relative effects is complicated because residential and non-residential vegetation can be correlated (Smith et al. 2005, Melles et al. 2003, VanHeezik et al. 2008). Indeed, depending on how they are measured, the areas of residential and non-residential vegetation may act as suppressor variables because they both have positive effects, yet they can be either positively correlated (e.g., the area of parks and the total area of residential tree cover, within a surrounding landscape, Melles et al. 2003) or negatively correlated (e.g., areas of exclusive landcovers within a fixed-radius landscape, Evans et al. 2009). Despite the correlations, they are generally managed independently. For example, in Ottawa the regulations controlling the area of parks and natural vegetation retained in a new development (City of Ottawa bylaw \#2009-95) are independent of the size of yards or the 
building density in the development, which are largely driven by market forces.

Similarly, there are separate regulations related to trees in residential (bylaw \#2009-200) and non-residential areas (bylaw \#2006-279) of the city. Therefore, because they are correlated and yet can be independently managed, it is important to estimate their relative effects in a way that is independent of collinearity and suppressor effects (Smith et al. 2009).

Partial regression coefficients from a regression of bird diversity on the area and structure of residential and non-residential vegetation are the best estimate of their relative effects. The area of residential and non-residential vegetation and the percent of that area covered by trees or shrubs (as an index of vegetation structure) are simple variables that have plausible, causal influences on bird diversity (see following paragraphs). In addition, they are separately represented by existing planning laws (see above). Therefore, they represent competing management options and, assuming plausible confounding effects are controlled (Anderson et al. 2001), their partial coefficients from a multiple regression can be interpreted as estimates of likely change in bird diversity that could be expected from independently managing each factor (Chapter 4).

The relative effects of residential and non-residential vegetation may be scale dependent if the relevant mechanisms that link vegetation with biodiversity vary with scale (Addicot et al. 1987, Smith et al. in press). At a local scale (i.e., within the area surveyed for bird-diversity), because both residential and non-residential vegetation act as habitat for native species (Jokimaki and Suhonen 1998), the species area relationship should lead to increasing native species richness with increasing areas of both 
(Rosenzweig 1995, McKinney 2008). Alternatively, biotic homogenization, where residential vegetation tends to support more non-native species and exclude some native ones (McKinney 2006), may mean that the local effects on native species richness may be weaker for residential vegetation than for non-residential. Similarly, because it is more highly fragmented (roads and buildings throughout), negative edge effects such as decreased nest success and increased disturbance (Marzluff and Ewing 2001), may reduce the overall quality of residential vegetation for most species. Therefore, increasing its area should have weaker effects than a similar increase in the area of non-residential vegetation

At the landscape-scale (i.e., surrounding the area surveyed), different mechanisms may be relevant. Increasing residential and non-residential vegetation in the surrounding landscape may have similar positive effects because either should decrease isolation (Schmiegelow and Monkkonen 2002). Alternatively, isolation effects may be much stronger for non-residential vegetation because it is generally much less structurally connected than residential vegetation (Gaston et al. 2005). Finally, landscape complementarity may result in stronger effects of non-residential vegetation, because the surrounding landscape can include large patches of non-residential vegetation, which support species that are not present in smaller patches or in residential vegetation and that use resources within the residential areas (Colding 2007).

The relative importance of managing residential and non-residential vegetation area may be different from their per-area relative effects, if there are different practical or theoretical limits to their management (Grace and Bollen 2005). Social and economic forces impose limits on the range of potential manipulations that may be different for the 
areas of residential and non-residential vegetation. For example, there are economic incentives for the developer of a new suburban development that separately affect: the size of each yard, the footprint of the house, and the amount of the developed area that is reserved for public parks and greenspace. Although the relative importance of managing the two types of vegetation is unknown, some studies have explicitly guessed at their relative importance; suggesting that managing for biodiversity in residential areas is likely too politically difficult to be useful (e.g., Chapman and Reich 2007). Similarly, most conservation recommendations for urban areas focus on non-residential areas, which is an implicit assumption about relative importance (Marzluff and Ewing 2001, Environment Canada 2007). However, without quantitative estimates of the relative potential benefit to conservation from managing residential and non-residential vegetation, these sorts of priority judgements may be premature.

Proportional partial coefficients (Chapter 4), which account for the practical or theoretical limits to management, provide an explicit, transparent, and quantitative estimate of the relative importance of managing residential and non-residential vegetation. Proportional partial coefficients are a data-independent standardization or rescaling, which allow partial coefficients to be compared on a common scale that considers the range of possible management limits. This proportional re-scaling gives greater weight to factors that have wider limits to management and lesser weight to those that are more constrained. Increasing human population density is one of the strongest limiting factors in urban development (Nilsson and Florgard 2009) and may impose different limits on the area of residential and non-residential development. For a given area of vegetation, the practical limitations to manipulating the percent of that vegetation 
that is trees or shrubs are likely similar in residential and non-residential areas, so their relative importance should be the same as their relative effects.

My objectives were to answer three questions. 1) What are the relative effects of residential and non-residential vegetation (area and percent covered in trees) on the species richness and the conservation value of an urban bird community? 2) Are the relative effects of residential and non-residential vegetation different at local and landscape scales? 3) What is the relative importance of managing the area of residential and non-residential vegetation, within the practical limits to management that are imposed by human population density?

\subsection{Methods}

\section{Study Area}

Ottawa, Ontario, Canada is an urban centre of approximately 800,000 residents, surrounded by a landscape composed of agricultural fields and coniferous-deciduous, mixed-wood forests. The city is within the Mixedwood Plains ecozone (Ecological Stratification Working Group 1996) and the Lower Great Lakes/St. Lawrence Plain, North American Bird Conservation Region, number 13 (hereafter BCR 13, Rich et al. 2004). Residential vegetation in Ottawa is primarily composed of mowed lawns, ornamental gardens, and a mix of deciduous and coniferous trees and shrubs. Nonresidential vegetation is a mix of: city parks, which include areas managed for natural vegetation as well as mowed lawns, planted trees, and sports fields; a large greenbelt surrounding the urban core, which includes large areas of forest, old pastures, wetlands, and agricultural fields and pastures; and, other large areas of primarily tall-grass and shrub vegetation, which includes verges along highways, vacant lots, and power-line rights of way. 


\section{Field Methods}

Bird observations were made by volunteers with the Ottawa Breeding Bird Count (OBBC) - a breeding season monitoring program for birds within the urban and suburban areas of the city of Ottawa, Ontario, Canada (www.ottawabirds.ca). OBBC surveys are conducted at randomly selected locations within an approximately $900 \mathrm{~km}^{2}$ study area that includes the city's urban core, a large greenbelt area composed of a mix of forests, wetlands, and agricultural fields, and the surrounding suburban developments that fall within the city boundaries. Observers used ten-minute, $75 \mathrm{~m}$, fixed-radius point counts, which were conducted under appropriate weather conditions and during the peak of the breeding season (the same conditions and season used by the North American Breeding Bird Survey, Peterjohn 1994), between 30 minutes before sunrise and 8:00am. Surveys were completed earlier in the day than many standard bird-monitoring protocols, to limit the influence of traffic and other human activity. If the count was located beside a road, observers also recorded the number of vehicles passing them during the ten-minute count. Each point count was conducted at a randomly selected, publicly-accessible location, separated from each other by at least $250 \mathrm{~m}$, and distributed so that there was one count location within each cell of a $1 \mathrm{~km} \times 1 \mathrm{~km}$ grid covering the entire study area. These publicly-accessible locations were primarily on sidewalks, roadsides, or city walking paths. Because this study was focused specifically on birds in residential areas, I selected 247 locations, from the full database of OBBC point counts, which fell within the areas designated as residential by the city's official plan (Figure 5.1, Appendix I). This selection removed any counts that fell within the rural parts of the city or commercial/industrial areas and included points that were sampled during the breeding seasons of 2007, 2008, or 2009. In cases where observations were available from more 
than 1 year, the most recent year's data were used. I did not combine data from multiple years at the same site because there were too few counts with multiple years of observations.

I tested for variation in bird detectability among observers and levels of traffic noise, to ensure this variation did not bias my results. I used removal models (Farnsworth et al. 2002) by dividing each ten-minute count into five, two-minute segments, during which only new bird observations were recorded. I used a Huggins, full closed captures with heterogeneity variation of the removal model, which allows for variation in the detectability among species (Huggins, 1989). I estimated the average, cumulative (across the full 10 minute point count period) proportion of the true number of species that were actually observed for each observer and for two levels of traffic (low and high traffic corresponding to points with fewer or more than 15 vehicles per ten minute count, respectively). Although my analyses in this paper use only 247 counts of the $>1000$ available in the $\mathrm{OBBC}$ database, I estimated detectability using the entire database, to increase precision. Detectability was analysed using the program Mark (White and Burnham 1999), though the R-package, RMark (Laake 2010).

\section{Measures of bird diversity and conservation value}

At each point count location, I calculated three measures of species richness and one index of conservation value. I calculated species richness for the following three groups: 1) native species (hereafter, S.Native), from which I excluded three native species that are often considered pests of urban environments because of their overabundance - Ring-billed Gulls (Larus delawarensis), Herring Gulls (Larus argentatus), and Canada Geese (Branta canadensis); 2) native, forest dependent species (hereafter, S.Forest); and 3) native, shrub-successional dependent species (hereafter, S.Shrub). Other 
habitat guilds (e.g. wetland, or grassland dependent species) were too rarely observed in my surveys to be modeled effectively. In addition to the three species richness measures, I included an index of conservation value (Nuttle et al. 2003), which weighted species abundances by a species-specific conservation priority value. Conservation priority values for each species were taken from the Partners in Flight (PIF), species assessment, threat scores, specific to the study region (i.e., scores for BCR 13, Panjabi et al 2005). I combined these species assessment scores with the bird observations at each point count, to create the following conservation index measure:

$$
\text { CI.Rank }=\sum_{j=1}^{S} P I F \cdot \text { Rank }_{j} \times\left(\left(\log _{10} n_{j}\right)+1\right) \quad \text { Equation } 1
$$

Where $n$ represents the number of individuals for species $j$ and PIF.Rank is the partners in flight priority ranking suggested by Nuttle et al. (2003), which combines the five PIF threat-scores (scores related to population size, population trend, threats to breeding, breeding distribution, and BCR area importance) into a single $0-5$ ranked scale. These abundances, weighted by threat score were summed over $S$, all species observed at each point count. Weighting the indices by species abundance on a log scale (as suggested by Herrando et al. 2010) is one way to account for relative abundance at a site, without over-prioritizing common species (e.g., 1 Sedge Wren contributes twice as much to the index as 10 Red-winged Blackbirds). Non-native species do not contribute to this index (i.e., PIF.Rank = 0, Nuttle et al. 2003).

\section{Vegetation}

Measures of vegetation cover and structure were calculated separately at two scales: local - within the $75 \mathrm{~m}$ radius point count plot; and landscape - within a $425 \mathrm{~m}$ buffer around the edge of the point count plot (i.e., a circle, $500 \mathrm{~m}$ in radius that excludes 
the plot area itself, Figure 5.1). The local measures were made using high resolution ( $20 \mathrm{~cm}$ pixel) aerial photographs (taken in 2008), in combination with data produced by the city of Ottawa's planning department that identifies all impervious surfaces (i.e., paved surfaces such as roads, parking-lots, and sidewalks, as well as the footprints of all buildings in the city). With these two data sources I estimated the area of water, tree canopy, shrubs, tall grass, and mowed grass. The other local covariate included in the analyses was the presence or absence of high-traffic roads within the count area. Hightraffic roads included any highways or major commuting routes but excluded the residential streets of quiet neighbourhoods.

Landscape measures were made by combining four base-layers created by the city's planning department. Layers for surface water, natural vegetation in nonresidential areas, and tree canopy coverage in residential areas, were based on aerial photography and ground-truthed with field observations. The natural vegetation layer was a 20-class ecological categorization that I reclassified into tree, shrub, and tall grass categories to match the local-scale data. The fourth base-layer was the city's impervious surfaces coverage (see above). I subtracted the non-overlapping coverage of the four base-layers as an estimate of the mowed grass coverage within the surrounding landscape. The result was a continuous layer with the same five categories as were measured in the local plots. These four base layers from the city's planning department were considered current up to 2007, but because urban areas develop at a high rate, I visually compared the base-layers with the 2008 aerial photography and updated the data in areas under active development. 
For both the local and the landscape measures, vegetation area was calculated as the sum of the tree, shrub, tall grass, and mowed grass areas. Throughout the remainder of the text, measures of vegetation area are referred to as "Veg" followed by modifiers indicating residential or non-residential, and the scale within which it was measured (e.g., VegRes75 $=$ area of local residential vegetation). For vegetation structure, the percent of wooded vegetation was the percent of the vegetation area made up of tree and shrub classes, and the percent of shrub vegetation was calculated using the area of shrub and tall grass (areas of tall grass were included here because there were often scattered small shrubs within them). These measures are referred to as "pWood" or "pShrub" respectively, followed by the same modifiers as were used for vegetation area (e.g., pWoodNonRes $425=$ percent of non-residential vegetation that is wooded).

\section{Statistical Modelling}

I used generalized linear models with a log link and Poisson error distribution (after checking for overdispersion) for all species richness responses (i.e., S.Native, S.Forest, and S.Shrub) and an identity link and Gaussian error distribution for CI.Rank. In order to account for spatial autocorrelation, I used spatial Eigenvectors as covariates in all models (Griffith and Peres-Neto 2006, Bivand et al. 2010). Eigenvectors were included that removed any significant $(\mathrm{p}<0.10)$ autocorrelation (measured by Moran's I) in the residuals of a regression of each response on the total area of local vegetation (all vegetation within the $75 \mathrm{~m}$ radius plot). All analyses were conducted in $\mathrm{R}$, version 2.10.1 (R Development Core Team 2009). 


\section{Relative effects of residential and non-residential vegetation}

I modeled the separate effects of residential and non-residential vegetation at local and landscape scales for both vegetation area and vegetation structure. The final models were as follows:

\section{Equation 2}

$$
\begin{aligned}
& \text { Richness or CI.Rank }=\text { VegNonRes } 75+\text { VegRes } 75+p \text { WoodRes } 75+ \\
& \text { pWoodNonRes } 75+\text { VegNonRes } 425+\text { VegRes } 425+ \\
& \text { pWoodRes } 425+\text { pWoodNonRes } 425+\text { HighTraffic + SpEigen }
\end{aligned}
$$

Equation 3

$$
\begin{aligned}
& \text { S.Shrub }=\text { VegNonRes } 75+\text { VegRes } 75+\text { pShrubRes } 75+ \\
& \text { pShrubNonRes } 75+\text { VegNonRes } 425+\text { VegRes } 425+ \\
& \text { pShrubRes } 425+\text { pShrubNonRes } 425+\text { HighTraffic }+ \text { SpEigen }
\end{aligned}
$$

where the first eight terms represent the effects of vegetation area and structure in residential and non-residential areas, at the two scales (see above) and the last two terms represent a categorical covariate indicating the presence or absence of a high traffic road (as an index of human activity that may influence bird diversity and could confound my analyses, Fernandez-Juricic and Telleria 2000) and the spatial Eigenvectors that account for spatial autocorrelation. Equation 2 was used to model all response variables, except the richness of shrub-successional species (S.Shrub), in which case, Equation 3, with the Shrub-based vegetation structure variables, was used. Initially, I also considered quadratic terms for all of the vegetation structure variables but dropped them from the modeling because the terms had no significant effect on any of the responses and neither the bivariate plots of the raw data nor the residuals of the models indicated there were 
non-linear effects of vegetation structure. I plotted and compared the coefficients and their $95 \%$ confidence intervals, scaled for a 10 unit change in the percent of the landscape that is residential or non-residential vegetation or a 10 unit change in the percent of the vegetation that is wooded.

I replicated all of my analyses for a smaller subset of the data, in which there was less correlation between the areas of residential and non-residential vegetation at the local scale (Table 5.1). I did this to increase my confidence that my results applied across the full range of predictor values and that they are not overly influenced by the correlation between residential and non-residential vegetation area. To create this less-correlated sub-sample, I removed all points where local residential or non-residential vegetation was greater than 1.0 ha (i.e., $56 \%$ of the total, 1.78 ha local plot area). The specific cut-off was a compromise between removing the correlation and retaining a sufficient sample size. I then removed an additional random sample of points where residential vegetation was high $(>0.7 \mathrm{ha})$ and non-residential vegetation was low $(<0.3$ ha) because this combination was highly over-represented. The resulting, less correlated dataset included 130 locations of the original 247 and reduced the correlation between residential and nonresidential vegetation area from -0.70 in the full dataset to -0.51 in the reduced dataset (Table 5.1).

\section{Relative importance of managing vegetation area}

To translate my modeled estimates of the relative effects of vegetation area into estimates of relative importance that account for some of the practical limits to management, I re-scaled the coefficients for vegetation area into proportional partial coefficients (Chapter 4). I used a 95\% quantile regression (Koenker 2005) to identify the upper limit of residential and non-residential vegetation area (as a percent of the 
landscape area) with increasing human population density across my study area. The human population data came from the 2006 national census (www.statcan.gc.ca). I used my $500 \mathrm{~m}$ landscapes to compare vegetation and human population because it is closer to the scale of a residential neighbourhood or development; and although the effects on bird diversity may change with scale, management of vegetation area is constrained to the scale at which developments are planned. I scaled the proportional partial coefficients for $10 \%$ of the range of management limits at a moderately high population density of 300 residents per hectare.

\section{$5.4 \quad$ Results Detectability}

The percent of species observed was similar and very high, across all volunteers; they ranged from $91-96 \%$ across the 7 volunteers whose observations were included in my sample of 247 point counts. More than $95 \%$ of the counts were conducted by 5

volunteers whose average detection probabilities were $\geq 95 \%$. I ran all analyses both with all the data and with a reduced dataset that excluded data from volunteers with $<95 \%$ detection probability; the plotted coefficients and confidence limits were indistinguishable. Although there was a weak effect of traffic on detection probability, I did not correct for traffic in the final analysis for three reasons: 1) the cumulative detection probabilities for high and low noise sites were $94.5 \%$ and $96.3 \%$ respectively (i.e., $<2 \%$ different), 2) there were count locations where volunteers had not recorded traffic, and therefore, including a correction for traffic would have reduced the overall sample size; and 3) using only the point counts for which I had traffic data, I ran the relative effects analyses with a term for traffic level - it was not statistically significant 
for any of the response variables, increased the model AIC, and had little effect on the coefficient estimates for the remaining predictors $(<5 \%$ change in coefficient value).

\section{Relative Effects of residential and non-residential vegetation}

In the local landscape, the effects of residential vegetation area were as strong as, or slightly stronger than, the effects of non-residential vegetation area (upper plots in Figure 5.2). By contrast, in the surrounding landscape, the effects of non-residential vegetation area were generally stronger than the effects of residential vegetation (lower plots in Figure 5.2). In general, the effects of non-residential vegetation were about half as strong in the surrounding landscape as they were at the local scale (i.e., per $10 \%$ change in the amount of the landscape made up of non-residential vegetation, the percent change in richness or CI.Rank was approximately half of what was expected for the same change at the local scale, Figure 5.2). By comparison, the effects of residential vegetation area in the surrounding landscape, were effectively absent (i.e., the coefficient estimates were near zero with large confidence limits). Overall the summed effects of residential vegetation area at local and landscape scales were very similar to the summed effects for non-residential vegetation area (i.e., adding the effects in upper and lower plots of Figure 5.2). The slightly stronger local effects of residential vegetation area with their weak landscape effects give a combined effect that is very similar to that from the slightly lower local but much stronger landscape effects of non-residential vegetation. The results for the less correlated sub-sample of data were essentially the same as for the full dataset - so are not shown here - except that due to the smaller sample size, the confidence intervals were larger and included zero for some coefficients.

The effects of vegetation structure ("pWood" and "pShrub" variables) were generally weaker and less certain than those of vegetation area and depended more on the 
choice of response variable (Figure 5.3). In general, the relative effects of residential and non-residential vegetation structure were unclear because they were either both very weak (e.g., S.Native, Figure 5.3) or highly uncertain (S.Shrub and Cl.Rank, Figure 5.3). However, at the local scale, the percent of wooded area in residential vegetation had a slightly stronger positive effect on S.Forest than non-residential vegetation. At the landscape scale, this positive effect of residential wooded area was not evident. In contrast, the positive effect (although weak and uncertain) of wooded area in nonresidential lands on S.Forest was consistent at both the local and the landscape scale (Figure 5.3). Finally, the strongest estimated effect of vegetation structure was for the percent of residential vegetation in the landscape that was shrub or long grass on S.Shrub $(\sim 17 \%$ increase in S.Shrub for a $10 \%$ increase in pShrubRes425, Figure 5.3). However, this effect had wide confidence limits $(-5 \%$ to $+35 \%)$.

\section{Relative importance of managing vegetation area}

The area of non-residential vegetation declines more quickly with increasing human population than the area of residential vegetation (Figure 5.4). Within my 500m landscapes, the $95 \%$ quantile of residential vegetation area declines by approximately $4 \%$ for an increase of 100 people/ha (dotted line in Figure 5.4). In contrast, the $95 \%$ quantile for non-residential vegetation area declines by $18 \%$ over the same increase in population density (solid line in Figure 5.4). Using these quanitles as upper limits to management, proportional partial coefficients for residential and non-residential vegetation area indicate that at high human population densities (300/ha), when the limits to nonresidential vegetation are more constrained, residential vegetation is clearly a more important determinant of bird diversity than non-residential vegetation (Figure 5.5). 


\subsection{Discussion}

\section{Relative effects of residential and non-residential vegetation}

Overall, there are comparable relative effects of residential and non-residential vegetation area on the richness and conservation value of an urban bird community. Combined across scales, the effects of residential vegetation area (i.e., the area of private yards around houses and apartments) were very similar to the combined local and landscape effects of non-residential area (i.e., parks and other large greenspaces). Therefore, developers and city planners can influence the bird community of an urban area by managing both the area of vegetation within residential developments and the area that is set-aside for parks and greenspaces.

Although their combined effects were similar, the relative effects of residential and non-residential vegetation area were scale dependent. At the local scale (i.e., within the area surveyed), the effects of residential vegetation, were just as strong as, or slightly stronger than, those of non-residential vegetation, for the same change in area. Therefore, the potentially negative effects of homogenization (McKinney 2008) or negative edge effects associated with fragmented residential vegetation (Marzluff and Ewing 2001) are not as relevant for the urban bird community at this scale as are the positive species-area effects of increasing vegetation area. Their similar local scale relative effects also suggest that residential vegetation is not necessarily of lower overall quality for most bird species, nor even for species of greater conservation value (Chase and Walsh 2006). If it was, the local effect of residential vegetation area should have been weaker than that for nonresidential, particularly for the index of conservation value; in fact, it was similar or slightly stronger. 
There are two non-exclusive explanations for the additional landscape effect of non-residential vegetation area (Addicott et al. 1987): first, non-residential vegetation is generally more isolated and less connected; and, second, in my study design, it is more likely to affect landscape complementation processes. Non-residential vegetation is generally more isolated because it tends to be configured in discrete patches with simple shapes, separated by roads and large areas of residential and other urban development; whereas residential vegetation is generally configured in smaller, more complex patches, usually only separated by roads and often connected above the roads by the canopies of street trees (Gaston et al. 2005). Therefore, the landscape effects of non-residential vegetation area may reflect the influence of isolation-related processes such as immigration or landscape supplementation (Dunning et al. 1992). In addition, increasing the scale from local measures to landscape measures of non-residential vegetation captures the influence of a different kind of vegetation - large, unfragmented patches of relatively natural vegetation. The same is not true of residential vegetation, which, by definition, does not include unfragmented patches. Large areas of relatively natural vegetation provide different structure and resources than residential vegetation (e.g., understory vegetation in forests, Donnelly and Marzluff 2004). Therefore, non-residential vegetation in the surrounding landscape likely increases the diversity of residential bird communities through landscape complementation processes (Dunning et al. 1992) by providing necessary resources to species that would otherwise be absent from urban areas (Fernandez-Juricic and Jokimaki 2001, Melles 2003, Donnelly and Marzluff 2004).

Although I did not identify a strong landscape effect of residential vegetation area, studies of non-residential areas (e.g., parks etc.) may still need to control for the effects of 
the surrounding residential vegetation. The residential vegetation surrounding a park influences the park's bird community through complementation (Colding 2007), reducing edge effects (Fernandez-Jurcic and Telleria 2000), and providing connectivity among parks (Fernandez-Jurcic 2000). In addition, because the effects of residential and nonresidential vegetation area are both positive and yet they are negatively correlated in a fixed area, they should act as suppressor variables (Smith et al. 2009). Therefore, statistical models that only consider the area of either residential or non-residential vegetation (e.g., considering only the area of parks and greenspace and ignoring residential vegetation) could underestimate its importance.

In addition, my results do not mean that residential and non-residential vegetation necessarily have similar effects on the gamma diversity of birds in the entire city. My study focused on the birds observed in residential areas, which have important intrinsic conservation value, provide cultural ecosystem services that increase quality of life (e.g., hearing bird-song and seeing birds at feeders), and provide urban residents with a connection to native biodiversity that will have longer-term consequences for political attitudes towards conservation (Miller et al. 2005, Turner et al. 2004). However, a city consists of more than the residential areas that I have sampled here and these other areas make distinct contributions to bird diversity (e.g., in Ottawa, there are two rivers and numerous natural and artificial wetlands that have distinct bird communities). Measuring their relative effects on the diversity of birds in the entire city would require comparisons across multiple cities. Currently, few such data exist but urban bird monitoring programs such as the OBBC and related programs in Tucson, Arizona (McCaffrey 2005, 
www.tucsonbirds.org) and Fresno, California (www.fresnobirds.org) may allow such a comparison in the near future.

I did not find clear evidence that increasing residential vegetation area replaced sensitive species of high conservation value with urban-tolerant species of low conservation value. There was essentially no difference in the response of native species richness and an index of community conservation value, which down-weights common species with stable populations and fewer threats to their breeding habitat (Nuttle 2003). Certainly, the strong effect of non-residential vegetation area in the surrounding landscape indicates that parks and large greenspaces have an important role to play in the conservation value of an urban bird community. However, residential vegetation has an important role to play; at a local scale, the conservation value of the bird community was slightly more dependent on the area of residential vegetation than it was on the area of parks and other large areas of more natural vegetation. Across a longer gradient in increasing urbanization intensity (i.e., from large reserves through to the urban core), some species of high conservation value may be lost from the local bird community and replaced with tolerant species that are generally of lower conservation value (Blair 1996, McKinney 2006). However, within an intermediate portion of the longer urbanization gradient (i.e., in residential neighbourhoods), parks and private yards do not act as points along that gradient; they both play an important role in creating a bird community with value to conservation.

Increasing the tree and shrub cover of residential vegetation has a positive effect on forest bird richness; however, in relation to the effects of changing area, the effects of vegetation structure were weak, uncertain, and depended more on the choice of response 
variable. Only the richness of forest birds showed a clear response to increasing tree and shrub cover; in this case, the patterns in scale dependency were similar to that for vegetation area (i.e., stronger local effects of residential that are not evident at the landscape scale). Although percent tree and shrub cover is a simple variable that can be easily measured and is reflected well by existing planning laws, it is also a very coarse measure which may not represent the complex structural features of urban vegetation that affect birds. For example, because I have measured vegetation from aerial photos, I have no information on the variation in vertical structure, which is an important factor for many bird species (Murgui 2007, White et al. 2005).

\section{Relative importance of managing vegetation area}

Not only does increasing residential vegetation area increase bird diversity and conservation value but at higher human population densities, managing residential vegetation area becomes even more important than managing parks or other large greenspaces. Although the per-hectare effects of residential and non-residential vegetation are similar, increasing human population density imposes stricter limits on non-residential vegetation than it does on residential vegetation area. Therefore, as population density increases, the management of residential vegetation area has a greater capacity to influence bird diversity.

Although I have only considered one potential limit to management, I feel that human population density is a reasonable limit to consider for two reasons: 1) it is one of the most important factors in planning urban development that has profound consequences for many environmental and social factors (Lawrence 2005); and 2) despite numerous studies showing negative effects of increasing human population density on biodiversity (reviewed for birds in Chase and Walsh 2006, Evans et al. 2009), it is 
unlikely that urban planners would lower human population density in cities, for conservation reasons (Farber 2005, Nilsson and Florgard 2009). Indeed, it would be undesirable, if it meant increasing urban sprawl.

The particular limits to managing vegetation area that I have identified here reflect the current development patterns in the city but they may not represent theoretical limits. Thoughtful planning and design of urban areas could conceivably create residential areas with high human population density and much higher levels of non-residential vegetation than currently exist in Ottawa. For example, conservation subdivisions (Arendt 2004) or clumped housing developments (Gagne 2009) would change the limits to managing nonresidential vegetation and my estimates of relative importance.

Overall my results imply that the native species richness and conservation value of an urban bird community has as much to gain from increasing the area of vegetation in private yards as there is from increasing the area of park or greenspace in an urban/suburban neighbourhood. The greater flexibility of residential vegetation area under increasing human population density suggests that new developments with both higher population density and greater vegetation area are possible. This pragmatic approach would reconcile the need to house people with the importance of providing opportunities for those people to interact with native biodiversity in their daily lives (Rosenzweig 2005). 


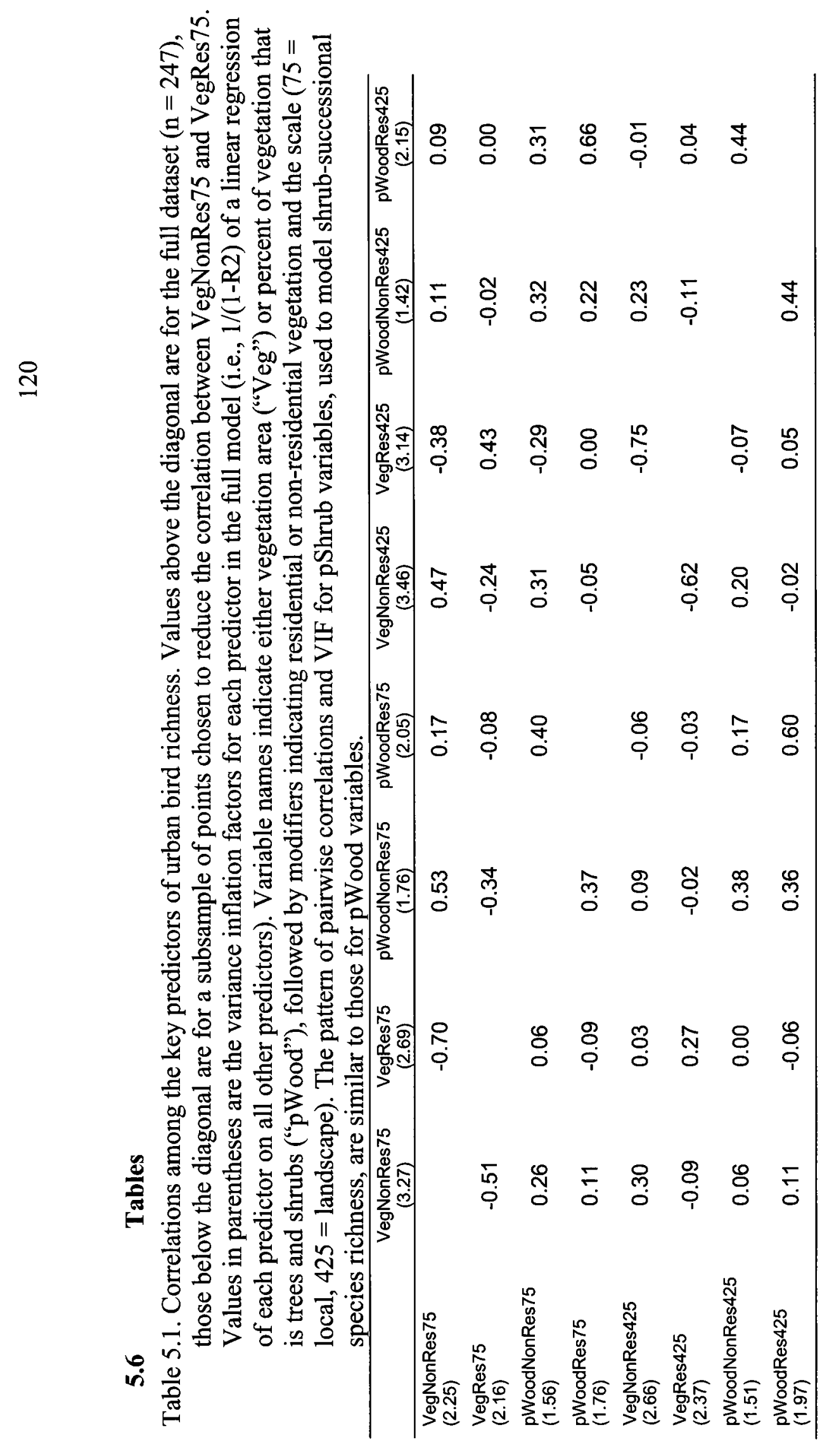




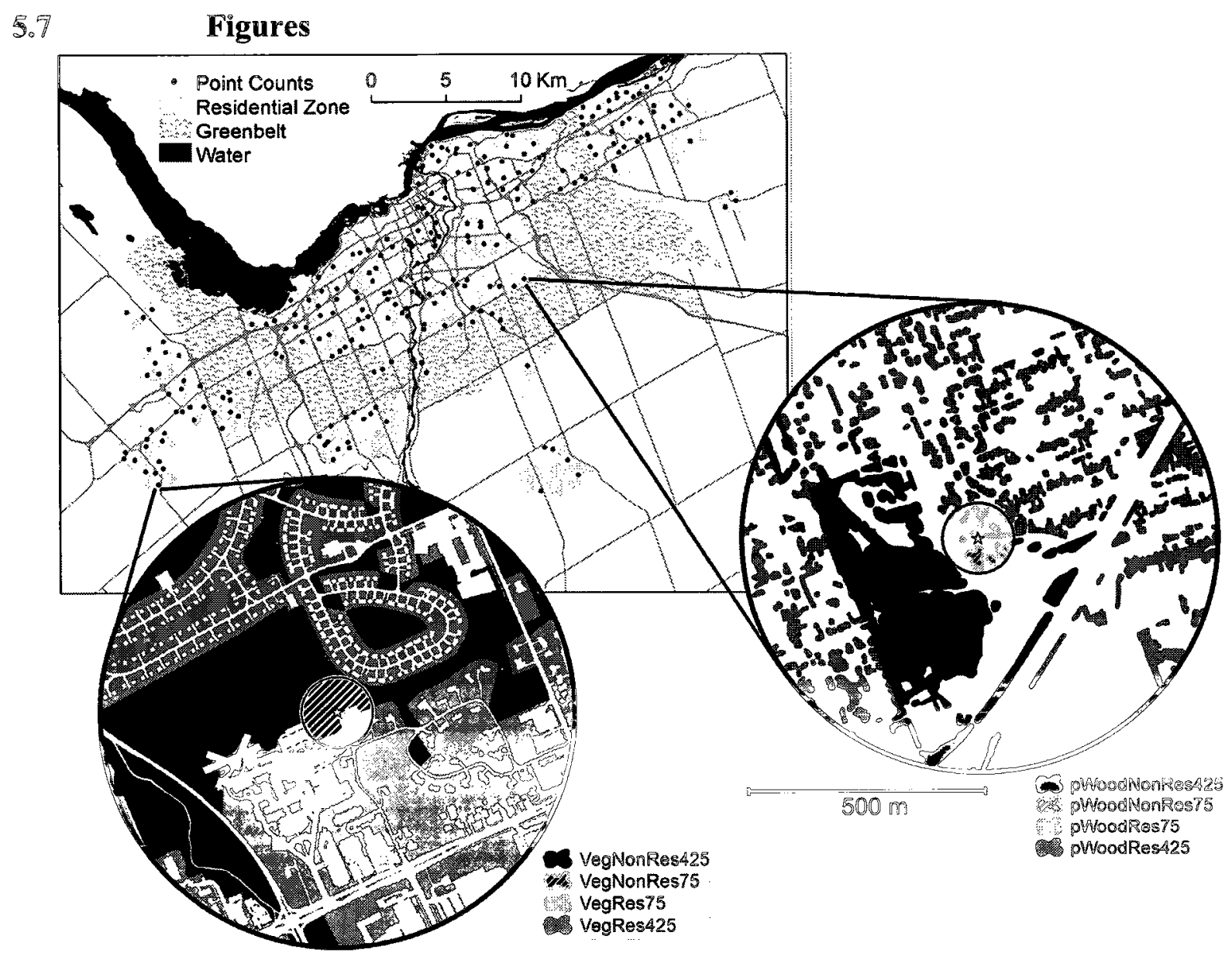

Figure 5.1. Map of study area in Ottawa, Ontario, Canada, showing the distribution of point counts across the city. Insets demonstrate how each of the four vegetation area (left inset), and four vegetation structure (percent of vegetation that is tree or shrub cover, right inset) variables were measured. See table 5.1 for a description of the variable names. 

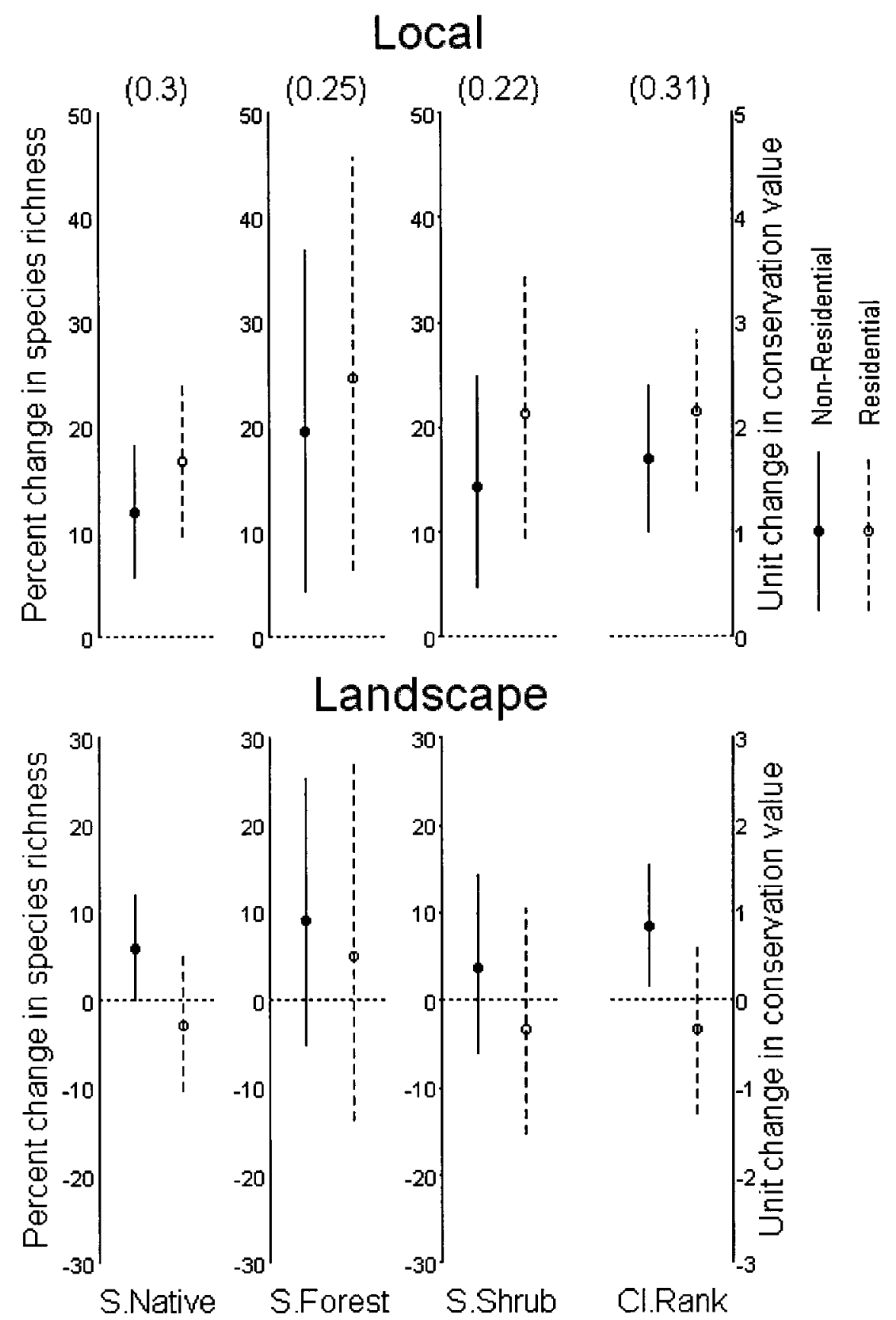

Figure 5.2. Relative effects of residential and non-residential vegetation area on four measures of bird diversity, at local (upper plots) and landscape (lower plots) scales. Points represent the partial coefficients for residential and non-residential vegetation area, scaled for a 10 unit change in the percent of the landscape made up of either residential or non-residential vegetation, after controlling for the remaining effects in Equation 2 or, for S.Shrub, Equation 3. Error bars represent $95 \%$ confidence intervals. Numbers in parentheses are the proportion of explained deviance for the model in Equation 2 or 3. 

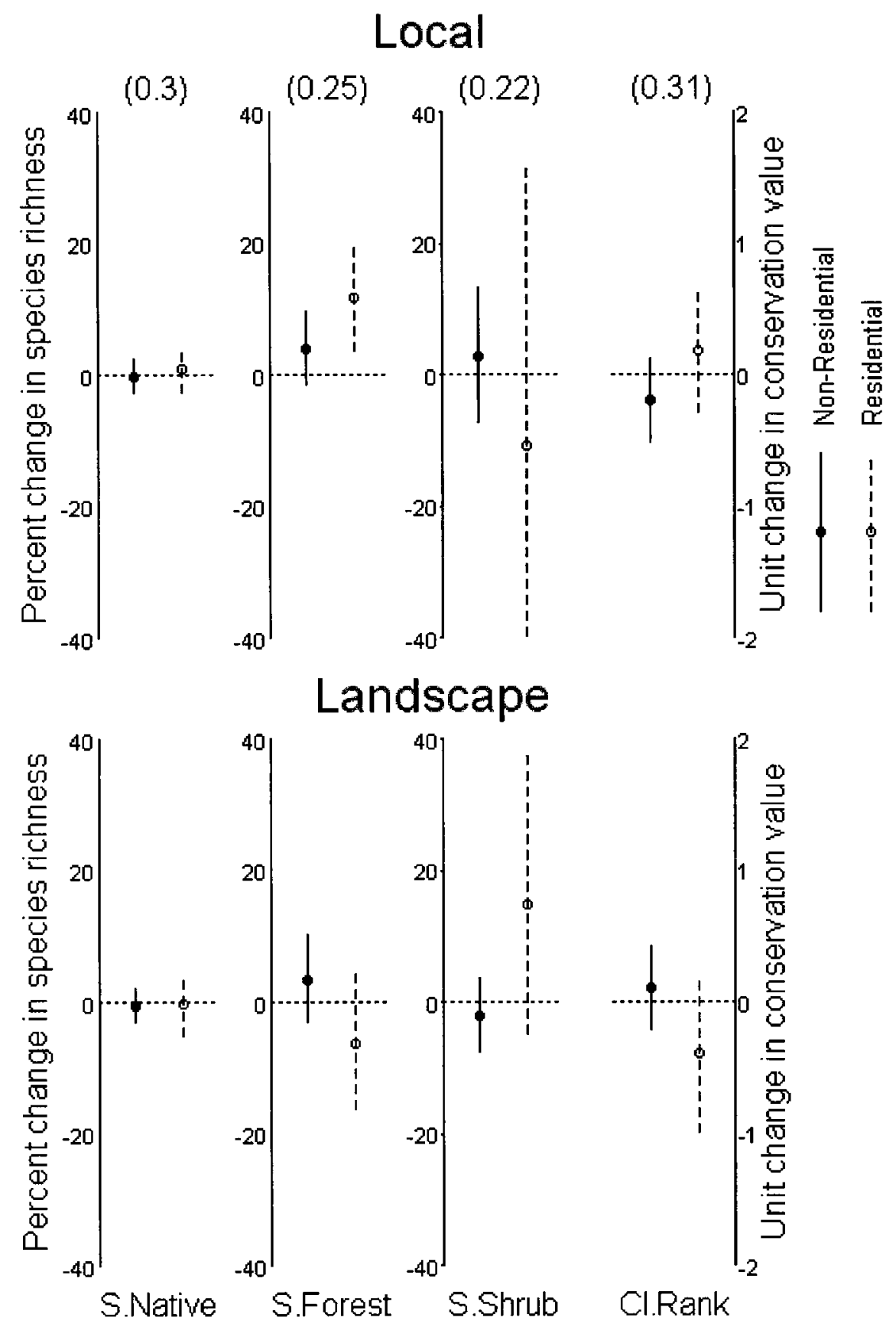

Figure 5.3. Relative effects of residential and non-residential vegetation structure (percent of vegetation that is wooded for S.Native, S.Forest, and CI.Rank, and the percent that is shrub or tall grass for S.Shrub) on four measures of bird diversity, at local (upper plots) and landscape (lower plots) scales. Points represent the partial coefficients, scaled for a 10 unit change in percent wooded or shrub, after controlling for the remaining effects in Equation 2 or for S.Shrub, Equation 3. Error bars represent $95 \%$ confidence intervals. Numbers in parentheses are the proportion of explained deviance for the model in Equation 2 or 3. 


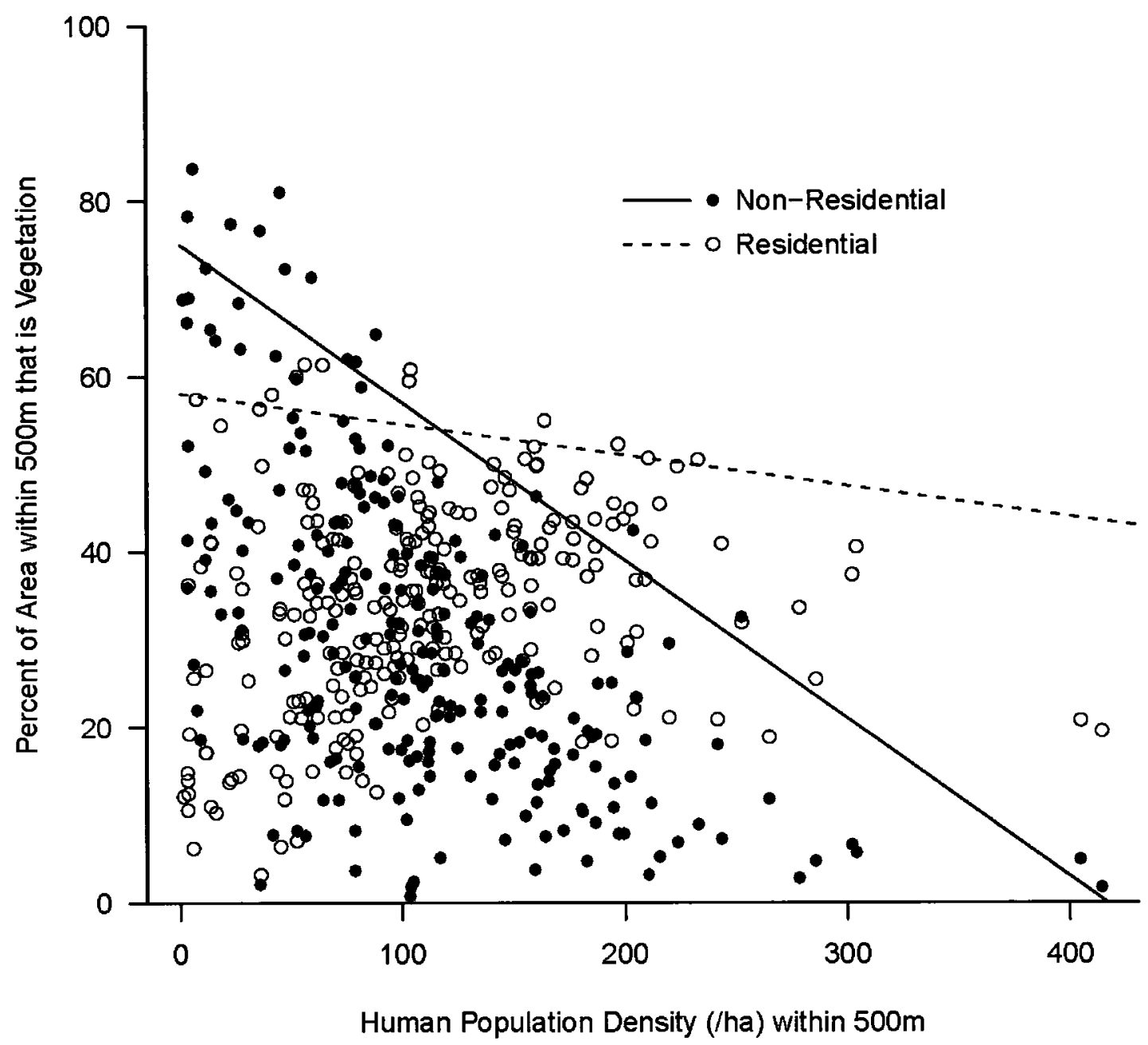

Figure 5.4. Limits to residential and non-residential vegetation area with increasing human population density. Lines represent $95 \%$ quantile regression models, fit separately to non-residential vegetation (solid line) and residential vegetation (dotted line). 

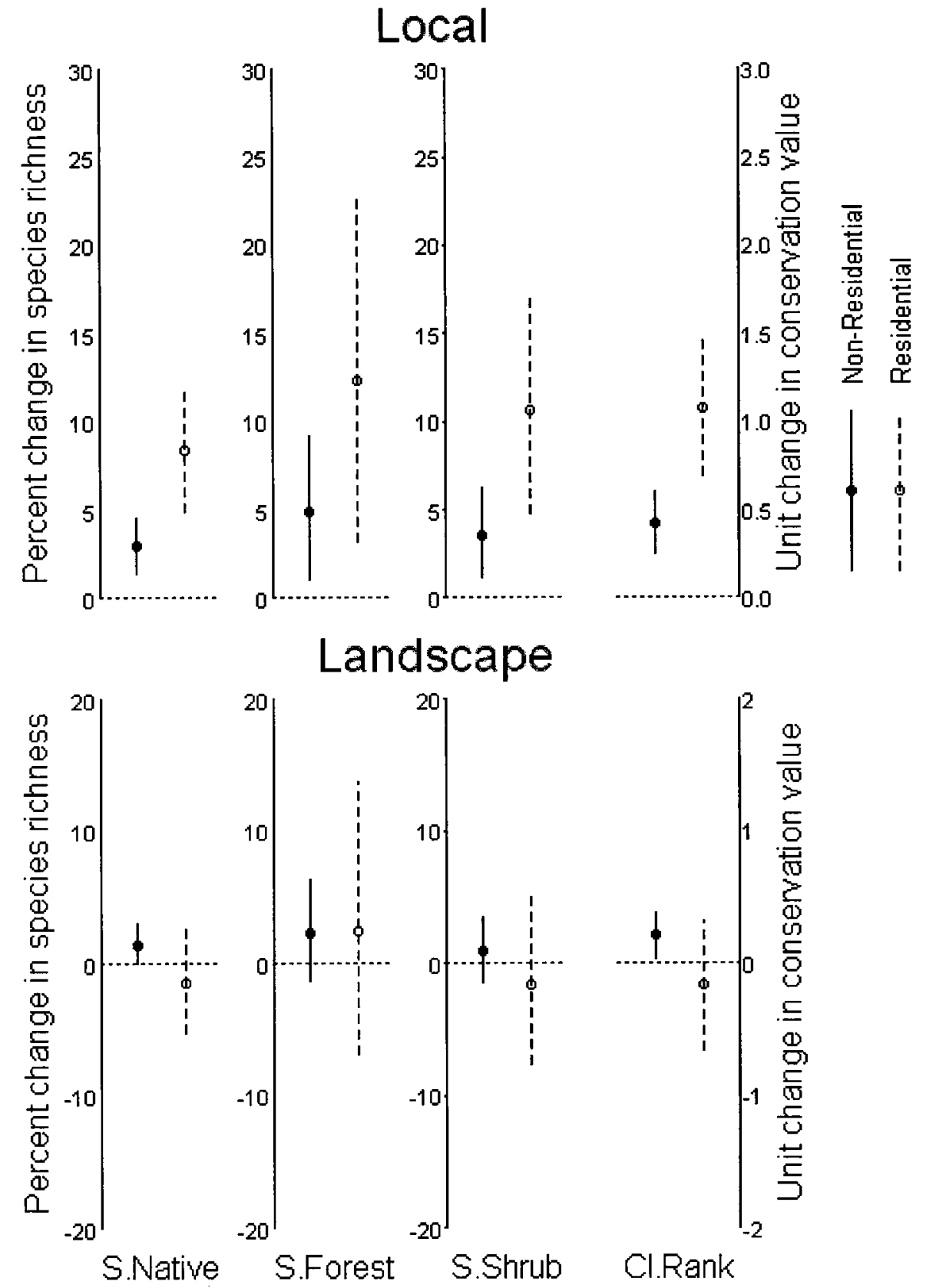

Figure 5.5. Relative importance of residential and non-residential vegetation at high human population density ( 300 residents/ha). Points represent the proportional partial coefficients, scaled to a change in vegetation area that is $10 \%$ of the range within practical limits to management. The limits to management were estimated from the ranges in the areas of residential and non-residential vegetation at high human population density (i.e., the point where the lines in Figure 5.5 at a density of 300). Compare with figure 5.2. Error bars represent $95 \%$ confidence intervals. 


\section{Chapter 6. General Conclusion}

It is 2010 , the international year of biodiversity, and there is a crisis in biodiversity. The 2010 target of the international convention on biodiversity - "to significantly reduce the current rate of biodiversity loss at the global, regional and national level as a contribution to poverty alleviation and to the benefit of all life on Earth" - has not been met. Indeed almost all indicators suggest that the plight of global biodiversity has worsened in the 8 years since the convention was ratified (Secretariat of the Convention on Biological Diversity 2006, Burton 2009).

Not coincidentally, I believe there is also a crisis in ecology and conservation biology. Our science is in crisis because we have often failed to provide useful information to managers, planners, politicians, and the public (Pullin and Knight 2009, Walpole et al. 2009). We fail when we preferentially study questions that lend themselves to rigidly defined hypothesis testing frameworks - e.g., tests of the existence of an effect

- when what is needed are estimates of relative importance - the relative potential benefit to, or effect on, biodiversity. Without the context of its effects relative to other factors, adding to the list of factors that significantly affect some component of biodiversity is unlikely to contribute to conservation. In fact, at its worst, it could distract from potentially more effective uses of limited conservation resources (Wilson et al. 2007). My thesis, I hope, is a small step in the right direction. Its overall message is to use the best available tools and evidence to estimate the relative strength of plausible effects and the confidence or uncertainty around them. For many practical questions in ecology and conservation biology, the best available tools and evidence are statistical models and observational data. A 20 year-old critique of ecology said as much: 
"Observational studies can only provide preliminary hypotheses that must be tested in manipulative experiments...manipulative experiments that are not conducted on the appropriate scale as the intended manipulation may prove trivial" (Peters 1991, pg. 140). For those questions where the appropriate scales are large in space and/or long in time, and where the "clean answers" and "critical tests" of strong inference are illusory (Platt 1964), these "preliminary hypotheses" may be the best option available within the short timeframes of applied management.

In Chapter 2, I have shown that the best method to estimate the relative effects of habitat amount and fragmentation, even when the predictors are highly correlated, is a standardized, partial regression coefficient from a plausible model where the potentially confounding factors have been controlled. Other statistical methods used to account for or remove collinearity are biased - in ways more complicated than has been previously acknowledged - and have probably done more harm than good. This work contributes to our understanding in landscape ecology in that: it highlights the literature about which we should be most skeptical, i.e., studies using residual regression and those that have not clearly reported correlations among predictors; and, it shows that the literature is not as biased towards the effects of habitat amount as was previously thought (Koper et al. 2007). Indeed, my findings here show that studies that have used residual regression may have underestimated the effects of both amount and fragmentation.

There are also clear methodological implications from this work. Studies of habitat amount and fragmentation, and landscape ecological analyses in general, should explicitly report collinearity among predictors - both pairwise correlations among the predictors and cumulative measures for each predictor, such as variance inflation factors. 
These correlations have a strong influence on uncertainty in the analysis and on any estimate of confidence such as confidence intervals, statistical significance, or Akaike weights (Neter et al. 1990, Burnham and Anderson 2002). By contrast, estimates of effect strength - standardized partial coefficients - are relatively insensitive to collinearity. In addition, this work shows that it is important to consider the direction of a predictor's effect in combination with its correlation to other predictors; the suppressor effects that I have shown here are unacknowledged in landscape ecological modeling, yet I suspect they have profoundly influenced the results of studies.

In Chapter 3, I have shown a scale dependency in the relative effects of forest amount and forest fragmentation on bird species that are considered sensitive to forest fragmentation. This scale dependence is largely due to the higher variability of fragmentation effects, across scales. The most important implication from this study is that habitat fragmentation effects may be sufficiently complex that we should be skeptical that managing fragmentation is at all useful. If species that are thought to be particularly sensitive to increasing fragmentation do not have consistent responses across scales, it is questionable whether there will be any positive effect of simple management prescriptions, such as increasing the core area of forests (Environment Canada 2003). It is important to note that my work has only examined forest birds in southern Ontario; other bird species (e.g., grassland birds - Hamer et al. 2006, or tropical forest birds - Laurance et al. 2002) or taxa may show more consistent responses to fragmentation across scales and/or species.

In Chapter 4, I have provided a framework and categorization of the numerous statistical metrics of relative importance as well as criteria by which to judge other 
metrics. I have also shown that the best statistical metric for prioritizing competing management options is a partial regression coefficient, re-scaled as a proportion of the practical or theoretical limits to management. This work represents a synthesis of theory and methods for linking statistical models to management actions for conservation. Methodologically, it builds on the more specific findings in Chapter 1 - in fact, it contradicts one of the recommendations in Chapter 1 - by comparing a wider range of metrics than any previous study on relative importance, and testing them against a priori criteria. It also introduces a new metric that compares predictors on a data-independent common scale (Grace and Bollen 2005), which explicitly acknowledges the practical or theoretical limits to management. This chapter also makes some novel theoretical contributions. It is the first study to propose a clear definition for relative importance in the context of applied studies in ecology and conservation - the relative potential benefit to biodiversity from independently managing each factor. It also represents the first study to propose criteria that any measure of relative importance in conservation should meet. Finally, it provides a framework that explains the characteristics (i.e., biases and sensitivities) of each of the three categories of metrics and the individual metrics themselves. Each of these is an important and novel contribution to ecology and conservation biology.

One implication of the findings in this chapter is that the benefits of estimating relative importance using regression parameters should apply equally well regardless of the particular modeling approach (Johnson 1995). For example proportional coefficients can be estimated using generalized linear models (McCullagh and Nelder 1989), generalized additive models (Hastie and Tibsharani 1990), hierarchical variance models 
(Gelman and Hill 2007), Bayesian approaches (McCarthy 2005), or structural equation modeling (Grace and Bollen 2005). Each of these methods relies on a different set of assumptions and a different model of the underlying ecological phenomenon but parameters on a proportional scale have clear implications for conservation in all cases.

Finally, one outstanding methodological and philosophical question in estimating relative importance is how to prioritize management options when factors interact (Healy 1990). In the case of an interaction, both factors have some importance but their relative effect strengths may vary. To date, this is an issue that has been almost entirely neglected in both the ecological and statistical literature.

In Chapter 5, I have shown that the relative effects of residential and nonresidential vegetation area on the richness and conservation value of an urban bird community are approximately equal at a local scale; however, at a landscape scale, the effects of non-residential vegetation area are greater. As human population density increases, the more restrictive limits imposed on non-residential vegetation mean that its overall importance declines in relation to the importance of managing residential vegetation.

The finding that residential and non-residential vegetation area are generally similar in their relative effects on urban bird diversity contrasts with the tone of most literature on the effects of urbanization (Chase and Walsh 2006 and McKinney 2008, although compare Evans et al. 2009). This finding implies that within cities, there may be more potential for managing urban bird communities than previously thought (Marzluff and Ewing 2001). It also implies that although in some situations, vegetation management may be politically more difficult in privately-owned residential land than in 
non-residential land, the relative potential benefits to biodiversity are at least similar; and, in areas of high population density the greater relative benefits of managing residential vegetation may counteract any potential political difficulty (Chapman and Reich 2007).

I believe my thesis, particularly Chapter 4, has some reasonably broad and profound implications for ecology and conservation biology. Overall, it implies that thoughtfully constructed, yet relatively simple, statistical models can provide valuable insight into the likely consequences of potential management actions. This is not a particularly profound finding on its own; however, the variable and disparate definitions of "relative importance" from which authors have made recommendations for conservation management, suggest that we have not been thoughtful enough in interpreting statistical models. I hope my thesis' theoretical contributions will stimulate deeper consideration of the appropriate links between statistical results and conservation implications in the literature.

I also hope that they will stimulate further discussion on the balance in conservation biology between the limits to our knowledge and the imperative to act (Hilborn and Ludwig 1993, Johnson 2002, Wiens et al. 2008). How confident can we be in applying the results of statistical models of observational data, even within the context of landscapes carefully selected to control for correlations and potential confounders (Brennan et al. 2002)? How can we optimize monitoring so that it is more likely to capture the natural experiments that can shed light on the likely impacts of manipulations? These natural experiments can help create the causal links between statistical predictors and biodiversity responses; however, they can be difficult to predict and so a great deal of monitoring data may be required to capture them (Nichols and 
Williams 2006). The philosophy and application of statistics in medicine may have much to offer ecology and conservation. Many medical problems and questions (e.g., the study of life-style factors on health) are similar to conservation problems in that it can be difficult to apply manipulative experiments and there are numerous potentially confounding effects. This literature may improve our ability to control for potentially confounding factors in the analysis of observational data (e.g., McNamee 2003).

My thesis highlights the importance of quantitative estimates of relative effects and relative importance in ecology and conservation, so that competing management options can be prioritized. Providing these quantitative estimates will require focusing our questions and our philosophical and analytical frameworks on biological significance and parameter estimation. We must remain conscious of uncertainty in those estimates and explicit in our reporting of that uncertainty, however, we cannot afford to let statistical and philosophical dogma interfere with asking difficult questions in complex systems where manipulative experiments are not feasible. If we want our science to influence society's actions, we must provide information that can be compared to the costs of applying various conservation actions. As ecologists and conservation biologists we must ask ourselves where the balance lies between the dangers of being wrong and having information that is good enough for now (Wiens 2009). 


\section{References}

Achen, C. H. 1982. Interpreting and using regression. Beverly Hills, CA: Sage.

Addicott, J. F., J.M. Aho, M.F. Antolin, D.K. Padilla, J.S. Richardson and D.A. Soluk. 1987. Ecological neighbourhoods: scaling environmental patterns. Oikos 49: 340346.

Adriaens, D., H. Jacquemyn, O. Honnay, and M. Hermy. 2009. Conservation of remnant populations of Colchicum autumnale - The relative importance of local habitat quality and habitat fragmentation. Acta Oecologica 35:69-82.

Anderson, D.R., K. P. Burnham, W. R. Gould, and S. Cherry. 2001. Concerns about finding effects that are actually spurious. Wildlife Society Bulletin 29:311-316

Andren, H. 1994. Effects of habitat fragmentation on birds and mammals in landscapes with different proportions of suitable habitat: a review. Oikos 71:355-366.

Arendt, R. 2004. Linked landscapes: Creating greenway corridors through conservation subdivision design strategies in the northeastern and central United States. Landscape and Urban Planning 68:241-269.

Austen, M. J. W., C. M. Francis, D. M. Burke, and M. S. W. Bradstreet. 2001. Landscape context and fragmentation effects on forest birds in southern Ontario. Condor 103: 701-714.

Balmford, A. J.L. Moore, T. Brooks, N. Burgess, L. A. Hansen, P. Williams, and C. Rahbek. 2001. Conservation conflicts across Africa. Science 291:2616.

Barbaro, L., J.P. Rossi, F. Vetillard, J. Nezan, and H. Jactel. 2007. The spatial distribution of birds and carabid beetles in pine plantation forests: the role of landscape composition and structure. Journal of Biogeography 34:652-664. 
Bartuszevige, A.M., D.L. Gorchov, and L. Raab. 2006. The relative importance of landscape and community features in the invasion of an exotic shrub in a fragmented landscape. Ecography 29:213-222.

Belisle, M., A. Desrochers, and M.J. Fortin. 2001. Influence of forest cover on the movements of forest birds: A homing experiment. Ecology 82:1893-1904.

Betts, M.G., G.J. Forbes, A.W. Diamond, and P.D. Taylor. 2006. Independent effects of fragmentation on songbirds in a forest mosaic. Ecological Applications 16:10761089.

Betts, M.G., G.J. Forbes, and A.W. Diamond. 2007. Thresholds in songbird occurrence in relation to landscape structure. Conservation Biology 21:1046-1058.

Bivand, R.L., R. Anselin, O. Assunção, A. Berke, E. Bernat, M. Blankmeyer, Y. Carvalho, B. Chun, C. Christensen, S. Dormann, R. Dray, E. Halbersma, N. Krainski, H. Lewin-Koh, J. Li, G. Ma, W. Millo, H. Mueller, P. Ono, G. PeresNeto, M. Piras, M. Reder, M. Tiefelsdorf and D. Yu. 2010. spdep: Spatial dependence: weighting schemes, statistics and models. R package version 0.4-58. $\underline{\text { http://CRAN.R-project.org/package }=\text { spdep }}$

Blair, R. 1996. Land use and avian species diversity along an urban gradient. Ecological Applications 6:506-519.

den Boer, P. J. 1981. On the survival of populations in a heterogeneous and variable environment. Oecologia 50: 39-53.

Boulinier, T., J.D. Nichols, J.R. Sauer, J.E. Hines, and K.H. Pollock. 1998. Estimating species richness: the importance of heterogeneity in species detectability. Ecology 79: 1018-1028. 
Bowman, J., N. Cappuccino, and L. Fahrig. 2002. Patch size and population density: the effect of immigration behavior. Conservation Ecology 6: 9 .

Brennan, J.M., D.J. Bender, T.A. Contreras, and L. Fahrig. 2002. Focal patch landscape studies for wildlife management: Optimizing sampling effort across scales. Pages 68-91 in: J. Liu and W. W. Taylor (eds.). Integrating landscape ecology into natural resource management. Cambridge University Press, Cambridge, MA.

Burke, D. M. and E. Nol. 1998. Influence of food abundance, nest-site habitat, and forest fragmentation on breeding ovenbirds. Auk 115:96-104.

Burke, D.M., K. Elliott, L. Moore, W. Dunford, E. Nol, J. Phillips, S. Holmes, and K. Freemark. 2004. Patterns of nest predation on artificial and natural nests in forests. Conservation Biology 18: 381-388.

Burnham, K.P. and D.R. Anderson. 2002. Model Selection and Multi-model Inference: A Practical Information-Theoretic Approach 2nd ed. Springer, New York.

Burton, A. 2009. 2010 biodiversity target won't be met. Frontiers in Ecology and the Environment 7:513-513.

Cade, B.S., J.W. Terrell, and R.L. Schroeder. 1999. Estimating effects of limiting factors with regression quantiles. Ecology 80:311-323.

Chalfoun, A.D., F.R. Thompson III and M.J. Ratnaswany. 2002. Nest predators and fragmentation: a review and meta-analysis. Conservation Biology 16: 306-318.

Chapman, K.A. and P.B. Reich. 2007. Land use and habitat gradients determine bird community diversity and abundance in suburban, rural and reserve landscapes of Minnesota, USA. Biological Conservation 135:527-541. 
Chase, J.F. and J.J Walsh. 2006. Urban effects on native avifauna. Landscape and Urban Planning 74:46-69.

Chevan, A., and M. Sutherland. 1991. Hierarchical partitioning. The American Statistician 45:90-96.

Clark, A.E. and C.G. Troskie. 2006. Regression and ICOMP - A Simulation Study. Communications in Statistics - Simulation and Computation 35(3):591 - 603.

Clergeau, P., J.P.L. Savard, G. Mennechez, and G. Falardeau. 1998. Bird abundance and diversity along an urban-rural gradient: A comparative study between two cities on different continents. Condor 100:413-425.

Cohen, J., and P. Cohen. 1983. Applied multiple regression/correlation analysis for the behavioral sciences. Lawrence Erlbaum, Mahwah, New Jersey, USA.

Colding, J. 2007. 'Ecological land-use complementation' for building resilience in urban ecosystems. Landscape and Urban Planning 81:46-55.

Cooper, C.B., and J.R Walters. 2002. Independent effects of woodland loss and fragmentation on Brown Treecreeper distribution. Biological Conservation 105:110.

Cushmam, S.A. and K. McGarrigal. 2003. Landscape-level patterns of avian diversity in the Oregon Coast Range. Ecological Monographs 73:259-281.

Daniels, G.D. and J.B. Kirkpatrick. 2006. Does variation in garden characteristics influence the conservation of birds in suburbia? Biological Conservation. 133:326-335.

Debuse, V.J., J. King, and A.P.N. House. 2007. Effect of fragmentation, habitat loss and within-patch habitat characteristics on ant assemblages in semi-arid woodlands of eastern Australia. Landscape Ecology 22:731-745. 
Deguise, I.E., and J.T. Kerr. 2006. Protected areas and prospects for endangered species conservation in Canada. Conservation Biology 20:48-55.

Dingle-Robertson, L. M. 2007. Land cover change mapping of eastern Ontario, 1995 to 2005 using object-based classification. M.Sc. Thesis. - Carleton University. Ottawa, Ontario.

Dodd, N.L., R.E. Schweinsburg, and S. Boe. 2006. Landscape-scale forest habitat relationships to tassel-eared squirrel populations: Implications for ponderosa pine forest restoration. Restoration Ecology 4:537-547.

Donnelly, R. and J.M. Marzluff. 2004. Importance of reserve size and landscape context to urban bird conservation. Conservation Biology 18:733-745.

Donnelly, R. and J.M. Marzluff. 2006. Relative importance of habitat quantity, structure, and spatial pattern to birds in urbanizing environments. Urban Ecosystems 9:99117.

Drolet, B., A. Desrochers, and M.J. Fortin. 1999. Effects of landscape structure on nesting songbird distribution in a harvested boreal forest. Condor 101:699-704.

Dunford, W. and K. Freemark. 2004. Matrix matters: effects of surrounding land uses on forest birds near Ottawa, Canada. Landscape Ecology 20:497-511.

Dunning, J.B., B.J. Danielson, and H.R. Pulliam. 1992. Ecological processes that affect populations in complex landscapes. Oikos 65: 169-75.

Ecological Stratification Working Group. 1996. A National Ecological Framework for Canada. - Agriculture and Agri-Food Canada, Research Branch, Ottawa/Hull, Canada. 
Environment Canada 2003 - How much habitat is enough? A Framework for Guiding Habitat Rehabilitation in Great Lakes Areas of Concern. available at www.on.ec.gc.ca/wildlife/publications-e.htm1

Environment Canada. 2007. Area-sensitive forest birds in urban areas. Ontario:

Environment Canada. URL http://www.on.ec.gc.ca/wildlife

Erikson, W.P., G.D. Johnson, and D.P. Young Jr. 2005. A Summary and Comparison of Bird Mortality from Anthropogenic Causes with an Emphasis on Collisions. - In: Ralph C. J. and Rich T. D. (eds), Bird Conservation Implementation and Integration in the Americas: Proceedings of the Third International Partners in Flight Conference. 2002 March 20-24; Asilomar, California, U.S. Department of Agriculture, Gen. Tech. Rep. PSW-GTR-191.

Evans K.L., S.E. Newson, and K.J. Gaston. 2009. Habitat influences on urban avian assemblages. Ibis 151:19-39.

Ewers, R. M. and R.K. Didham. 2006. Confounding factors in the detection of species responses to habitat fragmentation. Biological Reviews 81:117-142.

Ewers, R.M. and R.K. Didham. 2007. Habitat fragmentation: panchreston or paradigm? Trends in Ecology and Evolution 22:511.

Fahrig, L. 1997. Relative effects of habitat loss and fragmentation on population extinction. Journal of Wildlife Management 61:603-610.

Fahrig, L. 1998. When does fragmentation of breeding habitat affect population survival? Ecological Modelling 105:273-92.

Fahrig, L. 2002. Effects of fragmentation on the extinction threshold: A synthesis. Ecological Applications 12:346-353. 
Fahrig, L. 2003. Effects of habitat fragmentation on biodiversity. Annual Review of Ecology, Evolution and Systematics 34:487-515.

Farber, S. 2005. The economics of biodiversity in urbanizing ecosystems. In Eds.

Johnson E.A. and Klemens, M.W. Nature in Fragments: The Legacy of Sprawl. Columbia University Press. New York.

Farnsworth, G.L., K.H. Pollock, J.L. Nichols, T.R. Simons, J.E. Hines, and J.R. Sauer. 2002. A removal model for estimating detection probabilities from point-count surveys. The Auk 119:414-425.

Fernandez-Juricic, E. and J.L. Telleria. 2000. Effects of human disturbance on spatial and temporal feeding patterns of Blackbird Turdus merula in urban parks in Madrid, Spain. Bird Study 47:13-21.

Fernandez-Juricic, E. 2000. Avifaunal use of wooded streets in an urban landscape. Conservation Biology 14:513-521.

Fernandez-Juricic, E., and J. Jokimaki. 2001. A habitat island approach to conserving birds in urban landscapes: case studies from southern and northern Europe. Biodiversity Conservation 10:2023-2043.

Flather, C.H. and M. Bevers. 2002. Patchy reaction-diffusion and population abundance: The relative importance of habitat amount and arrangement. American Naturalist 159:40-56.

Fletcher, R.J. and R.R. Koford. 2002. Habitat and landscape associations of breeding birds in native and restored grasslands. Journal of Wildlife Management 66:10111022.

Freckleton, R.P. 2002. On the misuse of residuals in ecology: regression of residuals vs. multiple regression. Journal of Animal Ecology 71:542-545. 
Gagne, S.A. 2009. The effects of housing density and the trade-off between housing density and sprawl area on breeding birds and carabid beetles. Ph.D. Thesis Carleton University, 2009.

Gaston, K.J., P.H. Warren, K. Thompson, R.M. Smith. 2005. Urban domestic gardens (IV): the extent of the resource and its features. Biodiversity and Conservation $14: 3327-3349$.

Gelling, M., D.W. Macdonald and F. Mathews. 2007. Are hedgerows the route to increased farmland small mammal density? Use of hedgerows in British pastoral habitats. Landscape Ecology 22:1019-1032.

Gelman, A., and J. Hill. 2007. Data Analysis using Regression and Multilevel/Hierarchical Models. Cambridge University Press, NewYork.

Grace, J.B., and K.A. Bollen. 2005. Interpreting the results from multiple regression and structural equation models. Bulletin of the Ecological Society of America. $86: 283-295$

Graham, M. H. 2003. Confronting multicollinearity in ecological multiple regression. Ecology 84:2809-2815.

Griffith, D. A. and P.R. Peres-Neto. 2006. Spatial modeling in ecology: the flexibility of Eigenfunction spatial analyses. Ecology 87: 2603-2613.

Gromping, U. 2007. Estimators of Relative Importance in Linear Regression Based on Variance Decomposition. The American Statistician 61:139-147.

Guthery, F.S. 2008. A primer on natural resource science. Texas A\&M University Press, College Station, Texas. 
Guthery, F.S. 2008. Statistical ritual versus knowledge accrual in wildlife science. Journal of Wildlife Management 72:1872-1875.

Hamer, T.L., C.H. Flather, and B.R. Noon. 2006. Factors associated with grassland bird species richness: The relative roles of grassland area, landscape structure, and prey. Landscape Ecology 21:569-583.

Hastie, T. and R. Tibshirani. 1990. Generalized Additive Models. Chapman and Hall, Boca Raton, Florida, USA.

Healy, M.J.R. 1990. Measuring importance. Statistics in Medicine 9:633-637.

Hendrickx, F., J.P. Maelfait, W. van Wingerden, O. Schweiger, M. Speelmans, S. Aviron, I. Augenstein, R. Billeter, D. Bailey, R. Bukacek, F. Burel, T. Dieko“tter, J. Dirksen, F. Herzog, J. Liira, M .Roubalova, V. Vandomme, and R. Bugter. 2007. How landscape structure, land-use intensity and habitat diversity affect components of total arthropod diversity in agricultural landscapes. Journal of Applied Ecology 44:340-351.

Herrando S., L. Brotons, S. Guallar, and J. Quesada. 2010. Assessing regional variation in conservation value using fine-grained bird atlases. Biodiversity Conservation $19: 867-881$.

Hilborn, R. and D. Ludwig. 1993. The limits of applied ecological research. Ecological Applications 3:550-552.

Hilborn, R. and M. Mangel. 1997. The Ecological Detective: confronting models with data. Princeton University Press, Princeton, N.J.

Hobbs, N.T. and R. Hilborn. 2006. Alternatives to statistical hypothesis testing in ecology: a guide to self teaching. Ecological Applications 16:5-19. 
Hoover, J.P. and M.C. Brittingham. 1993. Regional variation in cowbird parasitism of wood thrushes. Wilson Bulletin 105: 228-238.

Hovel, K.A., and R.N. Lipcius. 2001. Effects of seagrass habitat fragmentation on juvenile blue crab survival and abundance. Journal of Experimental Marine Biology and Ecology 271:75-98.

Huggins, R.M. 1989. On the statistical analysis of capture experiments. Biometrika 76: $133-140$.

Hurlbert, S. H. 1984. Pseudoreplication and the design of ecological field experiments. Ecological Monographs 54:187-211.

James, F.C., and C.E. McCulloch. 1990. Multivariate analysis in ecology and systematics: panacea or Pandora's box? Annual Review of Ecology and Systematics 21:129-166.

Johnson, D.H. 1995. Statistical sirens: the allure of nonparametrics. Ecology 76:19982000.

Johnson, D.H. 1999. The insignificance of statistical significance testing. Journal of Wildlife Management 63:763-772.

Johnson, D.H. 2002. The importance of replication in wildlife research. Journal of Wildlife Management 66:919-932.

Johnson, J.J., and J.M. LeBreton. 2004. History and use of relative importance indices in organizational research. Organizational Research Methods 7:238-257.

Jokimaki, J. and J. Suhonen. 1998. Distribution and habitat selection of wintering birds in urban environments. Landscape and Urban Planning 39:253-263. 
Kareiva, P. 1990. Population dynamics in spatially complex environments: theory and data. Philosophical Transactions of the Royal Society of London 330:175-190.

Koenker, R.W. 2005. Quantile Regression, Cambridge U. Press.

Koper, N., F.K.A. Schmiegelow and E.H. Merrill. 2007. Residuals cannot distinguish between ecological effects of habitat amount and fragmentation: implications for the debate. Landscape Ecology 22:811-820.

Krawchuk, M.A. and P.D. Taylor. 2003. Changing importance of habitat structure across multiple spatial scales for three species of insects. Oikos 103:153-161.

Kruskal, W. 1987. "Relative Importance by Averaging over Orderings". The American Statistician 41:6-10.

Kruskal, W., and R. Majors. 1989. Concepts of Relative Importance in Recent Scientific Literature. The American Statistician 43:2-6.

Kupfer, J.A. Malanson, G.P., and Franklin, S.B. 2006. Not seeing the ocean for the islands: the mediating influence of matrix-based processes on forest fragmentation effects. Global Ecology and Biogeography 15:8-20.

Laake, J. 2010. RMark: R Code for MARK Analysis. R package version 1.9.6.

Lancaster, R.K. and W.E. Rees. 1979. Bird communities and the structure of urban habitats. Canadian Journal of Zoology-Revue Canadienne De Zoologie 57:23582368 .

Langlois, J.P., L. Fahrig, G. Merriam, and H. Artsob. 2001. Landscape structure influences continental distribution of hantavirus in deer mice. Landscape Ecology $16: 255-266$. 
Law, B.S. and C.R. Dickman. 1998. The use of habitat mosaics by terrestrial vertebrate fauna: implications for conservation and management. Biodiversity Conservation 7: $323-33$.

Laurance, W.F., T.E. Lovejoy, H.L. Vasconcelos, E.M. Bruna, R.K. Didham, P.C. Stouffer, C. Gascon, R.O. Bierregaard, S.G. Laurance, and E. Sampaio. 2002. Ecosystem decay of Amazonian forest fragments: a 22-year investigation. Conservation Biology. 16:605-618

Lawrence, B.L. 2005. The context and causes of sprawl. In Eds. Johnson E.A. and Klemens, M.W. Nature in Fragments: The Legacy of Sprawl. Columbia University Press. New York.

Legendre, P., and L. Legendre. 1998. Numerical ecology. Elsevier, Amsterdam, Netherlands.

Lichstein, J.W., T.R. Simons, and K.E. Franzreb. 2002. Landscape effects on breeding songbird abundance in managed forests. Ecological Applications 12:836-857.

Lindenmayer, D.B. and J. Fischer. 2007. Tackling the habitat fragmentation Panchreston. Trends in Ecology and Evolution 22:127-132.

MacKenzie, D.I., J.D. Nichols, J.A Royle, K.H. Pollock, L.L. Bailey, and J.E. Hines. 2006. Occupancy Estimation and Modeling: Inferring Patterns and Dynamics of Species Occurrence. - Elsevier, Burlington, MA.

MacNally, R. 2000. Regression and model-building in conservation biology, biogeography and ecology: The distinction between - and reconciliation of 'predictive' and 'explanatory' models. Biodiversity and Conservation 9:655-671. 
Magness, D.R., R.N. Wilkins, and S.J. Hejl. 2006. Quantitative relationships among golden-cheeked warbler occurrence and landscape size, composition, and structure. Wildlife Society Bulletin 34:473-479.

Marzluff, J.M. and K. Ewing. 2001. Restoration of fragmented landscapes for the conservation of birds: A general framework and specific recommendations for urbanizing landscapes. Restoration Ecology 9:280-292.

McAlpine, C.A., M.E. Bowen, J.G. Callaghan, D. Lunney, J.R. Rhodes, D.L. Mitchell, D.V. Pullar, H.P. Poszingham. 2006a. Testing alternative models for the conservation of koalas in fragmented rural-urban landscapes. Austral Ecology $31: 529-544$.

McAlpine, C.A., J.R. Rhodes, J.G. Callaghan, M.E. Bowen, D. Lunney, D.L. Mitchell, D.V. Pullar, and H.P. Poszingham. 2006b. The importance of forest area and configuration relative to local habitat factors for conserving forest mammals: A case study of koalas in Queensland, Australia. Biological Conservation 132:153165.

McCaffrey, R.E. 2005. Using Citizen Science in Urban Bird Studies. Urban Habitats 3:70-86.

McCarthy, M.A. 2007. Bayesian methods for ecology. Cambridge University Press, Cambridge, England.

McCullagh, P., and J.A. Nelder. 1989. Generalized linear models. 2 Ed. Chapman and Hall, New York, New York. 
McGarrigal, K. and S.A. Cushman. 2002. Comparative evaluation of experimental approaches to the study of habitat fragmentation effects. Ecological Applications 12: $335-345$.

McGarigal, K., S. A. Cushman, M. C. Neel, and E. Ene. 2002. FRAGSTATS: Spatial Pattern Analysis Program for Categorical Maps. Computer software program produced by the authors at the University of Massachusetts, Amherst. Available at the following web site: www.umass.edu/landeco/research/fragstats/fragstats.html

McGarrigal, K. and W.C. McComb. 1995. Relationships between landscape structure and breeding birds in the Oregon coast range. Ecological Monographs 65: 235-260.

McIntire, J.B.E. and A. Fajardo. 2009. Beyond description: the active and effective way to infer processes from spatial patterns. Ecology 90:46-56.

McKinney, M.L. 2006. Urbanization as a major cause of biotic homogenization. Biological Conservation 127:247-260.

McKinney, M.L. 2008. Effects of urbanization on species richness: A review of plants and animals. Urban Ecosystems 11:161-176.

McNamee, R. 2003. Confounding and confounders. Occupational and Environmental Medicine 60:227-234.

Melles, S., S. Glenn, and K. Martin. 2003. Urban bird diversity and landscape complexity: Species-environment associations along a multiscale habitat gradient. Conservation Ecology 7:22.

Miller, J.R. 2005. Biodiversity conservation and the extinction of experience. Trends in Ecology and Evolution 20:430-434.

Murgui, E. 2007. Factors influencing the bird community of urban wooded streets along an annual cycle. Ornis Fennica 84:66-77. 
Murray, K., and M.M. Conner. 2009. Methods to quantify variable importance: implications for the analysis of noisy ecological data. Ecology 90:348-355.

Neter, J., W. Wasserman, and M.H. Kutner. 1990. Applied linear statistical models, $3^{\text {rd }}$ ed. Irwin, Chicago, Illinois, USA.

Nichols, J.D., and B.K. Williams. 2006. Monitoring for conservation. Trends in Ecology and Evolution 21:668-673.

Nilsson, K.L. and C. Florgard. 2009. Ecological scientific knowledge in urban and landuse planning. In Eds. McDonnell, M.J., A.K. Hahs, and J.H. Breuste. Ecology of Cities and Towns: A Comparative Approach. Cambridge University Press.

Nol, E., C.M. Francis, and D.M. Burke. 2005. Using distance from putative source woodlots to predict occurrence of forest birds in putative sinks. Conservation Biology 19:836-844.

Norden, B., M. Ryberg, F. Gotmark, and B. Olausson. 2004. Relative importance of coarse and fine woody debris for the diversity of wood-inhabiting fungi in temperate broadleaf forests. Biological Conservation 117:1-10.

Nuttle, T., A. Leidolf, and L.W. Jr. Burger. 2003. Assessing conservation value of bird communities with partners in flight-based ranks. The Auk 20:541-549.

OBBA. 2001. Ontario breeding bird atlas guide for participants. Available online at: http://www.birdsontario.org/atlas/atlasmain.html.

OMNR. 1998. Ontario Land Cover Database. Ontario Ministry of Natural Resources, Peterborough Ontario, Canada.

OMNR. 2007. SOLRIS - Southern Ontario Land Information System. Ontario Ministry of Natural Resources, Peterborough, Ontario, Canada. 
Ontario Partners in Flight. 2005. Ontario Landbird Conservation Plan: Lower Great Lakes/St. Lawrence Plain (North American Bird Conservation Region 13), Priorities, Objectives and Recommended Actions.

Olson, G.S., E.M. Glenn, R.G. Anthony, E.D. Forsman, J.A. Reid, P.J. Loschl and W.J. Ripple. (2004). Modeling demographic performance of northern spotted owls relative to forest habitat in Oregon. Journal of Wildlife Management 68:10391053.

Panjabi, A.O., E.H. Dunn, P.J. Blancher, W.C. Hunter, B. Altman, J. Bart, C.J. Beardmore, H. Berlanga, G.S. Butcher, S.K. Davis, D.W. Demarest, R. Dettmers, W. Easton, H. Gomez de Silva Garza, E.E. Iñigo-Elias, D.N. Pashley, C.J. Ralph, T.D. Rich, K.V. Rosenberg, C.M. Rustay, J.M. Ruth, J.S. Wendt, and T.C. Will. 2005. The Partners in Flight handbook on species assessment. Version 2005. Partners in Flight Technical Series No. 3. Rocky Mountain Bird Observatory website: http://www.rmbo.org/pubs/downloads/Handbook2005.pdf

Pedhazur, E.J. 1997. Multiple regression in behavioral research. Third edition. Wadsworth, Belmont, California, USA.

Perhans, K., C. Kindstrand, M. Boman, L.B. Djupstrom, L. Gustafsson, L. Mattsson, L. M. Schroeder, J. Weslien, and S. Wikberg. 2008. Conservation Goals and the Relative Importance of Costs and Benefits in Reserve Selection. Conservation Biology 22:1331-1339.

Peterjohn, B.G. 1994. The North American Breeding Bird Survey. Birding 26:386-398. Peters, R.H. 1991. A critique for ecology. Cambridge University Press, Cambridge, England. 
Petraitis P.S., A.E. Dunham, and P.H. Niewiarowski. 1996. Inferring multiple causality: the limitations of path analysis. Functional Ecology 10:421-431.

Platt, J.R. 1964. Strong inference - Certain systematic methods of scientific thinking may produce much more rapid progress than others. Science 146: 347-353.

Popper, K. R. 1962. Conjectures and refutations. Basic Books, New York, New York, USA.

Porneluzi, P.A. and J. Faaborg. 1999. Season-long fecundity, survival, and viability of Ovenbirds in fragmented and unfragmented landscapes. Conservation Biology 13: 1151-1161.

Pratt, J.W. 1987. Dividing the indivisible: Using simple symmetry to partition variance explained. Pages 245-260 in T. Pukkila, and S. Puntanen, editors. Proceedings of second Tampere conference in statistics, University of Tampere, Finland.

Pullin, A.S. and T.M. Knight. 2009. Doing more good than harm: Building an evidencebase for conservation and environmental management. Biological Conservation 142: 931-934.

Quinn, J.F. and A.E. Dunham. 1983. On hypothesis testing in ecology and evolution. American Naturalist 122:602-617.

R Development Core Team. 2009. R: A language and environment for statistical computing. R Foundation for Statistical Computing, Vienna, Austria. ISBN 3900051-07-0, URL http://www.R-project.org.

Radford, J.Q, and A.F. Bennett. 2004. Thresholds in landscape parameters: occurrence of the white-browed treecreeper Climacteris affinis in Victoria, Australia. Biological Conservation 117:375-391. 
Radford, J.Q., and A.F. Bennett. 2007. The relative importance of landscape properties for woodland birds in agricultural environments. Journal of Applied Ecology 44:737-747.

Renfrew, R.B. and C.A. Ribic. 2008. Multi-scale models of grassland passerine abundance in a fragmented system in Wisconsin. Landscape Ecology 23: 181193.

Reunanen, P., A. Nikula, M. Monkkonen, E. Hurme, and V. Nivala. 2002. Predicting occupancy for the Siberian flying squirrel in old-growth forest patches. Ecological Applications 12:1188-1198.

Rich, T.D., C.J. Beardmore, H. Berlanga, P.J. Blancher, M.S.W. Bradstreet, G.S. Butcher, D.W. Demarest, E.H. Dunn, W.C. Hunter, E.E. I nigo-Elias, J.A. Kennedy, A.M. Martell, A.O. Panjabi, D.N. Pashley, K.V. Rosenberg, C.M. Rustay, J.S. Wendt, and T.C. Will. 2004. Partners in Flight North American Landbird Conservation Plan. Cornell Lab of Ornithology, Ithaca, NY, U.S.A.

Ricketts, T.H. 2001. The matrix matters: effective isolation in fragmented landscapes. American Naturalist 158: 87-99.

Ries, L. R.J. Fletcher Jr., J. Battin, and T.D. Sisk. 2004. Ecological responses to habitat edges: Mechanisms, models, and variability explained. Annual Review of Ecology, Evolution and Systematics 35:491-522.

Romesburg, H.C. 1981. Wildlife science - Gaining reliable knowledge. Journal of Wildlife Management 45:293-313. 
Rompre, G., W.D Robinson, A. Desrochers, and G. Angehr. 2007. Environmental correlates of avian diversity in lowland Panama rain forests. Journal of Biogeography 34:802-815.

Rosenberg, K.V., J.D. Lowe, and A.A. Dhondt. 1999. Effects of forest fragmentation on breeding tanagers: A continental perspective. Conservation Biology 13:568-583.

Rosenzweig, M.L. 1995. Species Diversity in Space and Time Cambridge Univ.Press, Cambridge, U.K.

Rosenzweig, M.L. 2005. Avoiding mass extinction: Basic and applied challenges. American Midland Naturalist. 153:195-208.

Schmiegelow, F.K.A, and M. Monkkonen. 2002. Habitat loss and fragmentation in dynamic landscapes: avian perspectives from the boreal forest. Ecological Applications 12:375-89.

Secretariat of the Convention on Biological Diversity (2006) Global Biodiversity Outlook 2. Montreal, Canada.

Simberloff, D. 1983. Competition theory, hypothesis-testing, and other community ecological buzzwords. American Naturalist 122:626-635.

Smith A.C., N. Koper, C.M. Francis, and L. Fahrig. 2009. Confronting collinearity: Comparing methods for disentangling the effects of habitat loss and fragmentation. Landscape Ecology 24: 1271-1285.

Smith A.C., C.M. Francis, and L. Fahrig. In Press. Landscape size affects the relative importance of habitat amount, habitat fragmentation, and matrix quality on forest birds. Ecography. 
Smith, R.M., K.J. Gaston, P.H. Warren, and K. Thompson. 2005. Urban domestic gardens (V): relationships between landcover composition, housing and landscape. Landscape Ecology. 20:235-253.

Stephens, P.A., S.W. Buskirk, G.D. Hayward, and C. Martmez del Rio. 2005. Information theory and hypothesis testing: a call for pluralism. Journal of Applied Ecology 42:4-12.

Stephens P.A., S.W. Buskirk and C. Martınez del Rio. 2007. Inference in ecology and evolution. Trends in Ecology and Evolution 22:192-197.

Stephens, S.E., J.J. Rotella, M.S. Lindberg, M.L. Taper, and J.K. Ringelman. 2005. Duck nest survival in-the Missouri Coteau of North Dakota: Landscape effects at multiple spatial scales. Ecological Applications 15:2137-2149.

Stinson, E.R., L.E. Hayes, P.B. Bush, and D.H. White. 1994. Carbofuran affects wildlife in Virginia corn fields. Wildlife Society Bulletin 22: 566-575.

Strong, D.R., L.A. Szyska, and D.S. Simberloff. 1979. Tests of community-wide character displacement against null hypotheses. Evolution 33: 897-913.

Sutherland W.J., A.S. Pullin, P.M. Dolman, and T.M. Knight. 2004. The need for evidence-based conservation. Trends in Ecology and Evolution 19:305-308.

Taki, H., P.G. Kevan, and J.S. Ascher. 2007. Landscape effects of forest loss in a pollination system. Landscape Ecology 22:1575-1587.

Thomas, D.R., E. Hughes, and B.D. Zumbo. 1998. On variable importance in regression. Social Indicators Research 45: 253-275. 
Tittler, R., L. Fahrig, and M.-A. Villard. 2006. Time-lagged correlations between Wood Thrush populations indicate long-distance dispersal and source sink dynamics. Ecology 87: 3029-3036.

Trzcinski, M.K., L. Fahrig, and G. Merriam. 1999. Independent effects of forest cover and fragmentation on the distribution of forest breeding birds. Ecological Applications 9: 586-593.

Turner M.G. 2005. Landscape ecology: What is the state of the science? Annual Review of Ecology Evolution and Systematics 36:319-344.

Turner, W.R., T. Nakamura, and M. Dinetti. 2004. Global urbanization and the separation of humans from nature. Bioscience 54:585-590.

Van Heezik, Y., A. Smyth. and R. Mathieu. 2008. Diversity of native and exotic birds across an urban gradient in a New Zealand city. Landscape and Urban Planning $87: 223-232$

Villard, M.A., M.K. Trzcinski, and G. Merriam. 1999. Fragmentation effects on forest birds: Relative influence of woodland cover and configuration on landscape occupancy. Conservation Biology 13:774-783.

Walpole, M., R.E.A. Almond, C. Besançon, S.H.M. Butchart, D. Campbell-Lendrum, G.M. Carr, B. Collen, L. Collette, N.C. Davidson, E. Dulloo, A.M. Fazel, J.N. Galloway, M. Gill, T. Goverse, M. Hockings, D.J. Leaman, D.H.W. Morgan, C. Revenga, C.J. Rickwood, F. Schutyser, S. Simons, A.J. Stattersfield, T.D. Tyrrell, J.-C. Vié, and M. Zimsky. 2009. Tracking Progress Toward the 2010 Biodiversity Target and Beyond. Science 325:1503-1504. 
Watson, M., N.J. Aebischer, G.R. Potts, and J.A. Ewald. 2007. The relative effects of raptor predation and shooting on overwinter mortality of grey partridges in the United Kingdom. Journal of Applied Ecology 44:972-982.

Westphal, M.I., S.A. Field, A.J. Tyre, D. Paton and H.P. Possingham. 2003. Effects of landscape pattern on bird species distribution in the Mt. Lofty Ranges, South Australia. Landscape Ecology 18:413-426.

White, J.G., M.J. Antos, J.A. Fitzsimons, and G.C. Palmer. 2005. Non-uniform bird assemblages in urban environments: the influence of streetscape vegetation. Landscape and Urban Planning 71: 12-135.

White, G.C. and K.P. Burnham. 1999. Program MARK: Survival estimation from populations of marked animals. Bird Study 46 Supplement, 120-138.

Wiens, J.A., G.D. Hayward, R.S. Holthausen, and M.J. Wisdom. 2008. Using surrogate species and groups for conservation planning and management. Bioscience $58: 241-252$.

Wiens, J.A. 2009. Uncertainty and the relevance of ecology. Bulletin of the British Ecological Society March, 2009. The British Ecological Society.

Wilson, K.A., E.C. Underwood, S.A. Morrison, K.R. Klausmeyer, W.W. Murdoch, B. Reyers, G. Wardell-Johnson, P.A. Marquet, P.W. Rundel, M.F. McBride, R.L. Pressey, M. Bode1, J.M. Hoekstra, S. Andelman, M. Looker, C. Rondinini, P. Kareiva, M.R. Shaw, and H.P. Possingham. 2007. Conserving biodiversity efficiently: What to do, where, and when. PLoS Biology 5:1850-1861. 
Wood, P.B., S.B. Bosworth, and R. Dettmers. 2006. Cerulean warbler abundance and occurrence relative to large-scale edge and habitat characteristics. Condor 108:154-165.

Yates, M.D. and R.M. Muzika. 2006. Effect of forest structure and fragmentation on site occupancy of bat species in Missouri ozark forests. Journal of Wildlife Management 70:1238-1248.

Yoccoz, N.G. 1991. Use, overuse, and misuse of significance tests in evolutionary biology and ecology. Bulletin of the Ecological Society of America 72:106-111. 


\section{Appendix I}

Appendix I. The year and location of 247 Ottawa Breeding Bird Count point counts used in the analyses of Chapter 5.

\begin{tabular}{|c|c|c|c|}
\hline OBBC Point Count & Year & UTM Nad83 Easting & UTM Nad83 Northing \\
\hline $10-2$ & 2007 & 456905 & 5031367 \\
\hline $10-3$ & 2007 & 456653 & 5031530 \\
\hline $10-4$ & 2007 & 456154 & 5031065 \\
\hline $10-6$ & 2007 & 455357 & 5031933 \\
\hline $10-7$ & 2007 & 455195 & 5030693 \\
\hline $11-1$ & 2008 & 453782 & 5032796 \\
\hline $11-2$ & 2008 & 454061 & 5032983 \\
\hline $12-2$ & 2008 & 453404 & 5033746 \\
\hline $12-4$ & 2008 & 452009 & 5034312 \\
\hline $12-5$ & 2008 & 451620 & 5034379 \\
\hline $12-6$ & 2008 & 451583 & 5033570 \\
\hline $12-8$ & 2008 & 450112 & 5032879 \\
\hline $13-1$ & 2008 & 452769 & 5033065 \\
\hline $13-2$ & 2008 & 451283 & 5032571 \\
\hline $13-3$ & 2008 & 449654 & 5032436 \\
\hline $13-4$ & 2008 & 449163 & 5033707 \\
\hline $13-5$ & 2008 & 448237 & 5033410 \\
\hline $13-6$ & 2008 & 448172 & 5033105 \\
\hline $14-2$ & 2008 & 448338 & 5031787 \\
\hline $14-3$ & 2008 & 449037 & 5031682 \\
\hline $14-4$ & 2008 & 450274 & 5032050 \\
\hline $14-5$ & 2008 & 451399 & 5031458 \\
\hline $14-6$ & 2008 & 451347 & 5030648 \\
\hline $14-7$ & 2008 & 452445 & 5031780 \\
\hline $14-8$ & 2008 & 453314 & 5031923 \\
\hline $15-1$ & 2009 & 450334 & 5031101 \\
\hline $16-10$ & 2009 & 449855 & 5028460 \\
\hline $16-11$ & 2009 & 449840 & 5028148 \\
\hline $16-9$ & 2009 & 451732 & 5027501 \\
\hline $17-1$ & 2009 & 450917 & 5026912 \\
\hline $17-2$ & 2009 & 450063 & 5026986 \\
\hline $17-3$ & 2009 & 449032 & 5027102 \\
\hline $17-4$ & 2009 & 448970 & 5027486 \\
\hline $17-6$ & 2009 & 448939 & 5028372 \\
\hline $17-9$ & 2009 & 449385 & 5030658 \\
\hline $18-1$ & 2008 & 447248 & 5033516 \\
\hline $18-2$ & 2008 & 446545 & 5033606 \\
\hline $18-3$ & 2008 & 447025 & 5032594 \\
\hline
\end{tabular}




\begin{tabular}{|c|c|c|c|}
\hline $18-4$ & 2008 & 446598 & 5033003 \\
\hline $18-6$ & 2008 & 445954 & 5032242 \\
\hline $18-7$ & 2008 & 445587 & 5032063 \\
\hline $18-8$ & 2008 & 445687 & 5031060 \\
\hline $18-9$ & 2008 & 446561 & 5030410 \\
\hline $19-1$ & 2009 & 446781 & 5026848 \\
\hline $19-2$ & 2009 & 446740 & 5027218 \\
\hline $19-3$ & 2009 & 447077 & 5027472 \\
\hline $19-4$ & 2009 & 446845 & 5028422 \\
\hline $19-5$ & 2009 & 447461 & 5030091 \\
\hline $19-6$ & 2009 & 447380 & 5031209 \\
\hline $20-1$ & 2007 & 446224 & 5029023 \\
\hline $20-2$ & 2009 & 445965 & 5029412 \\
\hline $20-3$ & 2007 & 445624 & 5029485 \\
\hline $22-1$ & 2007 & 443593 & 5023262 \\
\hline $22-2$ & 2007 & 443389 & 5023643 \\
\hline $22-3$ & 2007 & 443717 & 5023735 \\
\hline $22-5$ & 2007 & 443935 & 5026804 \\
\hline $22-6$ & 2007 & 443593 & 5027323 \\
\hline $22-7$ & 2007 & 445643 & 5027883 \\
\hline $22-8$ & 2007 & 446520 & 5027733 \\
\hline $23-2$ & 2009 & 444432 & 5024561 \\
\hline $23-3$ & 2009 & 445255 & 5023779 \\
\hline $23-4$ & 2009 & 444803 & 5022626 \\
\hline $23-5$ & 2009 & 444906 & 5022100 \\
\hline $31-4$ & 2008 & 466981 & 5029868 \\
\hline $31-5$ & 2008 & 466672 & 5030432 \\
\hline $31-8$ & 2008 & 466292 & 5029446 \\
\hline $35-1$ & 2008 & 450469 & 5024097 \\
\hline $35-2$ & 2008 & 451100 & 5024096 \\
\hline $35-3$ & 2008 & 452087 & 5024105 \\
\hline $35-4$ & 2008 & 452742 & 5024606 \\
\hline $36-4$ & 2008 & 451316 & 5021245 \\
\hline $39-2$ & 2008 & 445626 & 5025689 \\
\hline $39-4$ & 2008 & 446207 & 5024648 \\
\hline $39-5$ & 2008 & 446374 & 5023581 \\
\hline $39-7$ & 2008 & 446718 & 5022959 \\
\hline $39-8$ & 2008 & 447356 & 5023644 \\
\hline $4-3$ & 2008 & 463833 & 5036358 \\
\hline $40-1$ & 2009 & 447983 & 5024559 \\
\hline $40-2$ & 2009 & 448237 & 5024233 \\
\hline $40-3$ & 2009 & 448767 & 5024132 \\
\hline $40-4$ & 2009 & 448922 & 5024553 \\
\hline
\end{tabular}




\begin{tabular}{|c|c|c|c|}
\hline $40-5$ & 2009 & 449309 & 5022651 \\
\hline $40-6$ & 2009 & 449297 & 5021717 \\
\hline $40-7$ & 2009 & 449842 & 5022451 \\
\hline $40-8$ & 2009 & 450594 & 5022241 \\
\hline $40-9$ & 2009 & 451154 & 5021819 \\
\hline $42-2$ & 2008 & 454099 & 5013205 \\
\hline $42-3$ & 2008 & 454647 & 5013077 \\
\hline $42-4$ & 2008 & 455625 & 5012329 \\
\hline $42-6$ & 2008 & 456334 & 5011326 \\
\hline $44-6$ & 2007 & 453859 & 5010239 \\
\hline $46-10$ & 2007 & 452944 & 5018707 \\
\hline $48-2$ & 2007 & 446174 & 5018248 \\
\hline $5-1$ & 2007 & 463196 & 5035799 \\
\hline $5-10$ & 2007 & 457200 & 5033543 \\
\hline $5-2$ & 2007 & 462649 & 5036358 \\
\hline $5-3$ & 2007 & 461425 & 5035922 \\
\hline $5-4$ & 2007 & 461473 & 5036319 \\
\hline $5-5$ & 2007 & 459790 & 5035472 \\
\hline $5-6$ & 2007 & 459365 & 5035143 \\
\hline $5-7$ & 2007 & 458664 & 5034599 \\
\hline $5-8$ & 2007 & 458793 & 5033755 \\
\hline $5-9$ & 2007 & 457545 & 5032997 \\
\hline $50-7$ & 2008 & 447412 & 5021470 \\
\hline $50-8$ & 2008 & 448411 & 5021535 \\
\hline $51-5$ & 2007 & 445821 & 5018940 \\
\hline $51-7$ & 2007 & 446023 & 5021205 \\
\hline $51-8$ & 2007 & 446002 & 5021546 \\
\hline $55-5$ & 2007 & 445018 & 5015897 \\
\hline $55-7$ & 2007 & 445016 & 5019793 \\
\hline $58-1$ & 2008 & 443494 & 5014968 \\
\hline $6-10$ & 2007 & 461046 & 5034302 \\
\hline $6-7$ & 2007 & 460357 & 5033864 \\
\hline $6-8$ & 2007 & 460581 & 5034147 \\
\hline $6-9$ & 2007 & 461136 & 5033996 \\
\hline $60-1$ & 2008 & 438993 & 5013959 \\
\hline $60-2$ & 2008 & 439530 & 5013595 \\
\hline $60-3$ & 2008 & 439370 & 5013370 \\
\hline $60-4$ & 2008 & 440220 & 5013098 \\
\hline $60-5$ & 2008 & 440907 & 5013568 \\
\hline $60-6$ & 2008 & 440982 & 5013197 \\
\hline $60-7$ & 2008 & 439922 & 5012393 \\
\hline $61-4$ & 2007 & 442772 & 5015950 \\
\hline $61-5$ & 2007 & 442435 & 5015520 \\
\hline
\end{tabular}




\begin{tabular}{|c|c|c|c|}
\hline $61-6$ & 2007 & 441970 & 5015122 \\
\hline $62-3$ & 2007 & 443843 & 5019020 \\
\hline $62-6$ & 2007 & 443253 & 5018489 \\
\hline $62-7$ & 2007 & 442665 & 5018319 \\
\hline $66-2$ & 2008 & 439752 & 5014667 \\
\hline $66-3$ & 2008 & 440779 & 5015271 \\
\hline $66-4$ & 2008 & 441297 & 5015603 \\
\hline $67-1$ & 2008 & 433599 & 5015365 \\
\hline $67-2$ & 2008 & 433056 & 5015631 \\
\hline $67-3$ & 2008 & 432000 & 5015587 \\
\hline $68-1$ & 2007 & 432571 & 5016607 \\
\hline $68-2$ & 2007 & 432982 & 5016998 \\
\hline $68-3$ & 2007 & 433047 & 5016427 \\
\hline $68-5$ & 2007 & 432502 & 5014525 \\
\hline $69-1$ & 2007 & 443620 & 5021994 \\
\hline $69-10$ & 2007 & 441269 & 5026308 \\
\hline $69-2$ & 2007 & 444558 & 5022321 \\
\hline $69-3$ & 2007 & 444111 & 5022896 \\
\hline $69-4$ & 2007 & 442600 & 5024465 \\
\hline $69-5$ & 2007 & 442388 & 5025022 \\
\hline $69-6$ & 2007 & 442826 & 5025339 \\
\hline $69-7$ & 2007 & 443123 & 5026198 \\
\hline $69-8$ & 2007 & 442313 & 5026590 \\
\hline $69-9$ & 2007 & 441777 & 5026389 \\
\hline $7-10$ & 2007 & 460605 & 5035203 \\
\hline $7-6$ & 2007 & 463941 & 5033914 \\
\hline $7-8$ & 2007 & 462717 & 5035348 \\
\hline $7-9$ & 2007 & 462103 & 5034900 \\
\hline $70-1$ & 2007 & 438960 & 5024758 \\
\hline $70-2$ & 2007 & 439933 & 5025478 \\
\hline $70-3$ & 2007 & 441172 & 5025155 \\
\hline $70-4$ & 2007 & 441476 & 5023814 \\
\hline $70-5$ & 2007 & 441622 & 5023337 \\
\hline $70-6$ & 2007 & 441925 & 5022544 \\
\hline $70-7$ & 2007 & 441644 & 5021884 \\
\hline $70-8$ & 2007 & 442299 & 5022060 \\
\hline $70-9$ & 2007 & 441886 & 5021279 \\
\hline $71-1$ & 2009 & 436797 & 5021296 \\
\hline $71-2$ & 2009 & 437416 & 5021414 \\
\hline $71-3$ & 2009 & 438113 & 5021351 \\
\hline $71-4$ & 2009 & 439065 & 5022161 \\
\hline $71-5$ & 2009 & 439912 & 5022331 \\
\hline $71-6$ & 2009 & 440163 & 5023246 \\
\hline
\end{tabular}




\begin{tabular}{|c|c|c|c|}
\hline $71-7$ & 2009 & 440186 & 5023966 \\
\hline $71-8$ & 2009 & 439182 & 5023836 \\
\hline $72-1$ & 2008 & 439316 & 5025678 \\
\hline $72-3$ & 2008 & 438308 & 5023200 \\
\hline $72-4$ & 2008 & 438920 & 5022809 \\
\hline $72-6$ & 2008 & 437371 & 5022242 \\
\hline $72-8$ & 2008 & 437091 & 5023209 \\
\hline $72-9$ & 2008 & 437351 & 5023988 \\
\hline $73-3$ & 2008 & 441825 & 5020074 \\
\hline $73-4$ & 2008 & 441189 & 5020508 \\
\hline $73-5$ & 2008 & 439660 & 5020858 \\
\hline $73-6$ & 2008 & 438997 & 5020593 \\
\hline 73-7 & 2008 & 437883 & 5020404 \\
\hline $74-1$ & 2008 & 440704 & 5020030 \\
\hline $74-2$ & 2008 & 440376 & 5019756 \\
\hline $74-3$ & 2008 & 439859 & 5019396 \\
\hline $75-1$ & 2008 & 431049 & 5017402 \\
\hline $76-1$ & 2008 & 436037 & 5021833 \\
\hline $76-2$ & 2008 & 435463 & 5021566 \\
\hline $76-3$ & 2008 & 434504 & 5021595 \\
\hline $76-4$ & 2008 & 434689 & 5022401 \\
\hline $76-5$ & 2008 & 433695 & 5022650 \\
\hline $77-4$ & 2007 & 429432 & 5015658 \\
\hline $77-5$ & 2007 & 429849 & 5015672 \\
\hline $77-6$ & 2007 & 430395 & 5015962 \\
\hline $77-7$ & 2007 & 431314 & 5016009 \\
\hline $77-8$ & 2007 & 430649 & 5015110 \\
\hline $78-1$ & 2007 & 427506 & 5018157 \\
\hline $78-2$ & 2007 & 428027 & 5018958 \\
\hline $78-3$ & 2007 & 428292 & 5018229 \\
\hline $78-5$ & 2007 & 429949 & 5017763 \\
\hline $78-6$ & 2007 & 429796 & 5017196 \\
\hline $78-7$ & 2007 & 428956 & 5016879 \\
\hline $79-1$ & 2008 & 432225 & 5023618 \\
\hline $79-2$ & 2008 & 432499 & 5023657 \\
\hline $8-1$ & 2007 & 459476 & 5033529 \\
\hline $8-2$ & 2007 & 459110 & 5033150 \\
\hline $8-3$ & 2007 & 458738 & 5032231 \\
\hline $8-4$ & 2007 & 458853 & 5031953 \\
\hline $80-2$ & 2008 & 427255 & 5022083 \\
\hline $80-3$ & 2008 & 427862 & 5022612 \\
\hline $81-8$ & 2009 & 428594 & 5020424 \\
\hline $82-6$ & 2007 & 427630 & 5019914 \\
\hline
\end{tabular}




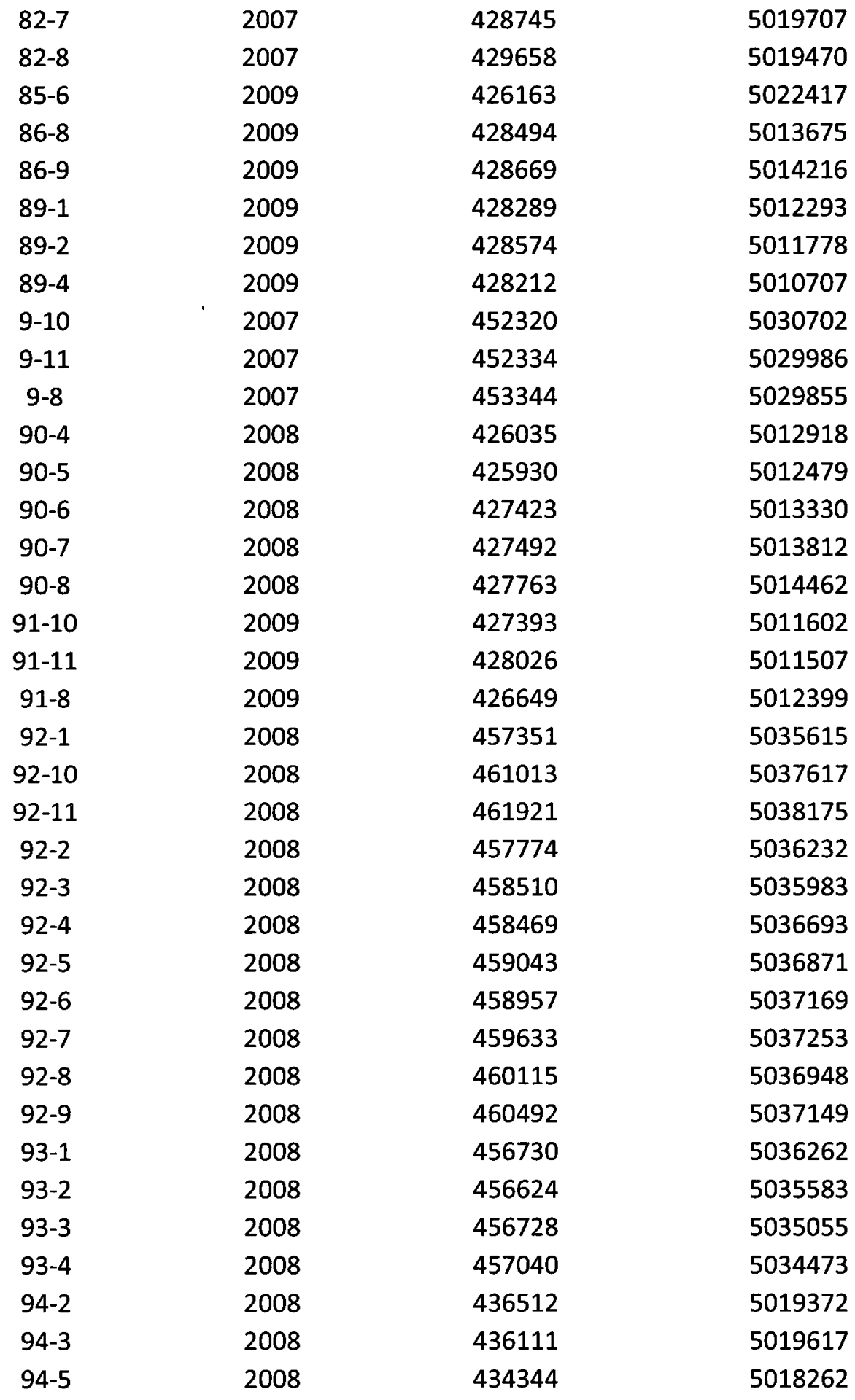

